\title{
Challenges and opportunities for multi-functional oxide thin films for voltage tunable radio frequency/microwave components
}

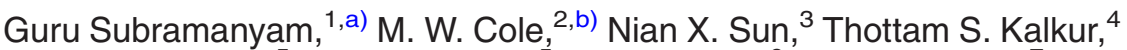 \\ Nick M. Sbrockey, ${ }^{5}$ Gary S. Tompa, ${ }^{5}$ Xiaomei Guo, ${ }^{6}$ Chonglin Chen, ${ }^{7}$ S. P. Alpay, ${ }^{8}$ \\ G. A. Rossetti, Jr., ${ }^{8}$ Kaushik Dayal, ${ }^{9}$ Long-Qing Chen, ${ }^{10}$ and Darrell G. Schlom ${ }^{11,12}$ \\ ${ }^{1}$ Department of Electrical and Computer Engineering, University of Dayton, Dayton, Ohio 45469, USA \\ ${ }^{2}$ U.S. Army Research Laboratory, Weapons and Materials Research Directorate, Aberdeen Proving Ground, \\ Maryland 21005, USA \\ ${ }^{3}$ Department of Electrical and Computer Engineering, Northeastern University, Boston, Massachusetts 02115 , \\ USA \\ ${ }^{4}$ Department of Electrical and Computer Engineering, University of Colorado, Colorado Springs, \\ Colorado 80918, USA \\ ${ }^{5}$ Structured Materials Industries, Inc., Piscataway, New Jersey 08854, USA \\ ${ }^{6}$ Boston Applied Technologies, Inc., Woburn, Massachusetts 01801, USA \\ ${ }^{7}$ Department of Physics \& Astronomy, University of Texas, San Antonio, Texas 78249, USA \\ ${ }^{8}$ Institute of Materials Science and Materials Science and Engineering Program, University of Connecticut, \\ Storrs, Connecticut 06269, USA \\ ${ }^{9}$ Mechanics, Materials and Computing, Civil and Environmental Engineering, Carnegie Mellon University, \\ Pittsburgh, Pennsylvania 15213, USA \\ ${ }^{10}$ Department of Materials Science and Engineering, Penn State University, University Park, \\ Pennsylvania 16802, USA \\ ${ }^{11}$ Department of Materials Science and Engineering, Cornell University, Ithaca, New York 14853, USA \\ ${ }^{12}$ Kavli Institute at Cornell for Nanoscale Science, Ithaca, New York 14853, USA
}

(Received 31 October 2012; accepted 14 May 2013; published online 15 November 2013)

\begin{abstract}
There has been significant progress on the fundamental science and technological applications of complex oxides and multiferroics. Among complex oxide thin films, barium strontium titanate (BST) has become the material of choice for room-temperature-based voltage-tunable dielectric thin films, due to its large dielectric tunability and low microwave loss at room temperature. BST thin film varactor technology based reconfigurable radio frequency $(\mathrm{RF}) /$ microwave components have been demonstrated with the potential to lower the size, weight, and power needs of a future generation of communication and radar systems. Low-power multiferroic devices have also been recently demonstrated. Strong magneto-electric coupling has also been demonstrated in different multiferroic heterostructures, which show giant voltage control of the ferromagnetic resonance frequency of more than two octaves. This manuscript reviews recent advances in the processing, and application development for the complex oxides and multiferroics, with the focus on voltage tunable $\mathrm{RF} /$ microwave components. The over-arching goal of this review is to provide a synopsis of the current state-of the-art of complex oxide and multiferroic thin film materials and devices, identify technical issues and technical challenges that need to be overcome for successful insertion of the technology for both military and commercial applications, and provide mitigation strategies to address these technical challenges. (C) 2013 AIP Publishing LLC. [http://dx.doi.org/10.1063/1.4827019]
\end{abstract}

\section{TABLE OF CONTENTS}

I. INTRODUCTION AND BACKGROUND .......

II. CURRENT STATE OF THE ART OF

MULTIFUNCTIONAL OXIDE AND

MULTIFERROIC DEVICES

\footnotetext{
${ }^{a)}$ Electronic mail: gsubramanyam1@udayton.edu

${ }^{b)}$ Electronic mail: melanie.w.cole.civ@mail.mil
}

A. Oxide thin film varactors ........... 3

B. Types of varactors .............. 3

C. Integrated varactor devices $\ldots \ldots \ldots \ldots \ldots \ldots$

D. Multiferroic thin film devices .......... 5

III. ADVANCES IN GROWTH-PROCESS SCIENCE METHODS ............... 6

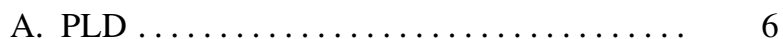

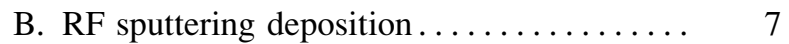

C. CSD .................... 9

D. MBE. ..................... 10

E. MOCVD.................. 10 
IV. TECHNOLOGY DRIVERS FOR OXIDE AND MULTI-FERROIC THIN FILMS FOR RF/MICROWAVE APPLICATIONS .........

A. Decoupling capacitors................

B. Passive reconfigurable RF/microwave

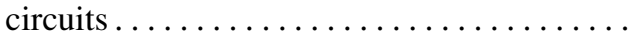

1. Tunable filters...................

2. Tunable bulk acoustic wave resonators with BST thin films ...............

3. Miniaturized reconfigurable antennas ...

C. Adaptive electronics based on tunable BST capacitors.......................

1. Impedance matching networks ........

2. VCOs .......................

D. Phase shifters for electronic steering of

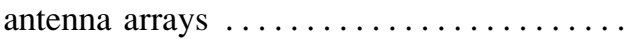

E. Multiferroic devices for technology drivers.

1. Voltage tunable magneto-electric RF/ microwave/millimeter wave devices ....

2. Low frequency sensors and sensor networks $\left(10^{-3}-10^{3} \mathrm{~Hz}\right) \ldots \ldots \ldots \ldots \ldots$

3. Broadband miniaturized antenna structures utilizing magneto-electric materials.......................

V. CHALLENGES FOR MULTIFUNCTIONAL OXIDE AND MULTIFERROIC THIN FILM

BASED TECHNOLOGIES.................

A. Material requirements for $\mathrm{RF} /$ microwave applications .....................

1. Effect of thickness uniformity of oxide thin films......................

2. Reducing dielectric loss-tangent in oxide thin films......................

3. Reducing the conductor loss for varactor devices.......................

4. Improving thermal stability in oxide thin films........................

5. Substrates and packaging considerations .

B. New techniques and heterostructures.......

C. Challenges for the multi-ferroic thin films ..

D. Challenges for modeling of complex oxide heterostructures ...................

VI. SUMMARY AND CONCLUSIONS ..........

\section{INTRODUCTION AND BACKGROUND}

Substantial strides have been made on bulk and single crystal complex oxide materials for an array of sensors and RF/microwave applications. Novel complex oxide and multiferroic materials are expected to have a significant impact on future sensor, communication, and surveillance systems. Therefore, complex oxide and multiferroic integrated thin film materials represent both an important and an enabling technology for a number of applications. At a fundamental level, complex oxide and multiferroic thin film materials are being developed at laboratories without a significant focus toward specific applications. This is a direct result of the minimal co-ordination between materials/device research groups and those with imminent needs in the application areas, thereby resulting in fewer opportunities for crossfertilization. This manuscript is an attempt to provide a comprehensive review for researchers on the technical issues related to application development utilizing thin films of multifunctional oxides and multiferroics. It is well known that the future system requirements drive towards smaller size, lighter weight, lower volume, minimal power consumption, and all of this combined with enhanced performance. These requirements cannot be met using conventional macroscopic "bulk materials" approaches and hence requires a refocus of research efforts toward innovative micro-nanoscale technologies, such as the emerging complex oxide and multiferroic thin film materials. Recent research indicates that thin film complex oxide and multiferroic materials provide unique opportunities to enable and subsequently field, innovative next-of-generation systems (e.g., radio frequency (RF)/microwave communication systems, guidance systems, antennas, uncooled IR sensors, biological/chemical detection, and novel energy systems, etc.). Integrated thin film complex oxide and multiferroic materials are expected to play a significant role in the development of these applications in the near future.

One of the key areas where multifunctional oxides and multiferroics can play a major role is in the RF and microwave systems. RF/microwave systems are increasingly broadband, leverage digital solutions, demand extremely high linearity, and seek to minimize size, weight, power, and cost (SWAP-C). Voltage tunable reconfigurable components utilizing multifunctional oxides and multiferroics can provide new capabilities including the possibility of simultaneous transmit and receive (STAR). Voltage control functionality of multiferroic devices can be devised with greater simplicity, efficiency, and robustness compared to magnetic control functionality. It is also less prone to cross-talk and interference effects compared to the magnetic interference effects. With this in mind, this manuscript will be limited to the complex oxides and multiferroics that are voltage/electric-field controllable. The use of voltage tunable passive components has attracted significant attention in the past two decades as RF and microwave engineers wish to have the flexibility of reconfigurable components so that their designs are not confined to a single frequency band of use. Complex communication systems including system on a chip (SOC) or a system in a package (SIP) would benefit tremendously if passive and active components can be reconfigured dynamically with ease, provide the option to use the system for multi-modes over a wide bandwidth, and support a variety of communication protocols. Such an adaptive, flexible, reconfigurable electronics is highly desirable for a $\mathrm{rf} /$ microwave engineer. Voltage tunable variable capacitors (varactors), variable inductors, and variable resistors (varistors) are of interest in the next generation reconfigurable multi-modal, multi-use communication systems as they could play a major role in dynamic impedance matching of power amplifiers (PAs), low-noise amplifiers (LNAs), reconfigurable antennas, voltage controlled oscillators (VCOs), broadband phase shifters for electronically steerable phased array systems, 
vector modulators, and other key components of communication and radar systems. These components will play a major role for realization of software defined radios and cognitive radios. ${ }^{1}$ These radio systems use a single hardware front-end and tune their frequency of operation, and operational bandwidth, based on software algorithms. ${ }^{1}$

In the subsequent sections of this manuscript, current state of the art (SOA) for thin films of multifunctional oxides and multiferroics is discussed in Sec. II, an overview of advances in processing of thin films of multifunctional oxides and multiferroics is provided in Sec. III; detailed discussions on various technology drivers for the voltage tunable devices are given in Sec. IV; major challenges and possible approaches to overcome the challenges are provided in Sec. V and finally conclusive statements, observations, and technology summary are provided in Sec. VI.

\section{CURRENT STATE OF THE ART OF MULTIFUNCTIONAL OXIDE AND MULTIFERROIC DEVICES}

\section{A. Oxide thin film varactors}

Variable capacitors (varactors) are utilized in microwave engineering for tunable filters, voltage controlled oscillators, phase shifter circuits, and other applications., ${ }^{2,3}$ Semiconductor varactor diodes, PIN diodes, RF MEMS varactors, and ferroelectric thin film varactors are potential candidates for varactor applications. Semiconductor varactor diodes and PIN diodes have relatively high Q below $10 \mathrm{GHz}$, but the $\mathrm{Q}$ degrades drastically above $10 \mathrm{GHz}$ making them less attractive for applications above $10 \mathrm{GHz}$. RF MEMS varactors offer high $\mathrm{Q}$ at microwave and millimeter wave frequencies, but are complex in nature, and the slow speed of switching is undesirable for many applications. Barium Strontium Titanate: $\mathrm{Ba}_{\mathrm{x}} \mathrm{Sr}_{1-\mathrm{x}} \mathrm{TiO}_{3}$ (henceforth BST) thin films have been investigated for room temperature tunable or reconfigurable RF and microwave components and circuits over the past 20 years. Majority of the studies on BST have utilized $\mathrm{Ba}_{0.6} \mathrm{Sr}_{0.4} \mathrm{TiO}_{3}$ or $\mathrm{Ba}_{0.7} \mathrm{Sr}_{0.3} \mathrm{TiO}_{3}$ compositions as they possess critical temperatures $\left(\mathrm{T}_{\mathrm{c}}\right)$ at or near room temperature. Recent developments on tunable dielectrics have shown that the varactors made of complex oxide BST thin films have constant Q through millimeter wave frequencies. ${ }^{4}$ BST thin film varactor devices are fast, small, and lightweight and, because they work using an electric field, have low power consumption. BST films exhibit an excellent tunable behavior at room temperature (in the case of $40 \%-60 \%$ concentration of barium), which lays the foundation for the microwave tunable devices. ${ }^{5,6}$ The range of capacitance tuning in BST varactors is quite large ( $>3: 1$ in the linear region) and varactor devices are relatively simple in nature. Also, BST thin film based varactors have exhibited high power handling capability which is also attractive for applications in microwave and millimeter-wave communications. ${ }^{7}$ Another advantage of the BST varactors is that they can handle both positive and negative voltages compared to only negative bias that can be applied to semiconductor varactor devices. The advantages of BST include dc voltage tunable high permittivity, high capacitance density (10-100 $\mathrm{nF} / \mathrm{mm}^{2}$ ), bipolar dc bias for tuning the varactor, large capacitance tuning range $(>3: 1)$, high power handling capability $(>1 \mathrm{~W})$, good temperature stability from $0^{\circ} \mathrm{C}$ to $110^{\circ} \mathrm{C}$ in thin films compared to bulk oxides, and quality factor in the frequency range of $1-10 \mathrm{GHz}$ comparable to semiconductor varactor diodes. Applications demonstrated using BST thin film varactors include tunable filters, ${ }^{8}$ tunable phase shifters, ${ }^{9}$ delay lines, ${ }^{10} \mathrm{VCO}^{11}$ and reconfigurable antennas. ${ }^{12}$ Table I compares the BST thin film varactor technology with semiconductor diode and the RF MEMS capacitor technologies.

\section{B. Types of varactors}

Two types of varactors have been considered for applications to tunable components and circuits. One is the coplanar interdigitated capacitor and the other the parallel plate capacitor. Figure 1 shows both the varactor designs. The parallel plate capacitors have been widely studied for tunable microwave device for low voltage and low power applications.

The interdigitated capacitor requires a single metal layer deposited on the BST thin film, and hence it is a simpler device to fabricate. The electric field between the fingers tunes the relative dielectric constant of the BST thin film. Since

TABLE I. Comparison of thin film BST varactor technology with semiconductor diodes and RF MEMS varactors. Please note that the typical BST thin film is only $0.4 \mu \mathrm{m}$ or lower.

\begin{tabular}{lccc}
\hline \hline Device characteristics and performance parameter & Semiconductor diodes & RF MEMS capacitors & Ferroelectric varactor (parallel plate) \\
\hline Tuning DC voltage & Low $(<10$ V reverse bias $)$ & High $(40-50$ V bipolar $)$ & Low $(<10 \mathrm{~V}$ bipolar bias $)$ \\
Capacitance tuning ratio & $>3: 1$ in the linear range & $>5: 1$ in the linear range & $\sim 3: 1$ in the linear range \\
Tuning speed & High $(\sim 10 \mathrm{~ns})$ & Low $(\sim 10 \mu \mathrm{s})$ & High $(\sim 10 \mathrm{~ns})$ \\
Quality factor $(\mathrm{Q})$ & $\sim 200$ at $1 \mathrm{GHz}$ & $>500 \mathrm{at} 1 \mathrm{GHz}$ & $\sim 200$ at $1 \mathrm{GHz}(0 \mathrm{~V})$ \\
Switching lifetime & High & Low & High \\
Packaging cost & Low & Very high & Low \\
Power handling & Poor $(\sim 1 \mathrm{~W})$ & Good $(<5 \mathrm{~W})$ & Good $($ up to $5 \mathrm{~W})$ \\
Power consumption & Low $(\mathrm{nW}-\mu \mathrm{W})$ & Low $(\mathrm{nW}-\mu \mathrm{W})$ & Low $(\mathrm{nW}-\mu \mathrm{W})$ \\
Breakdown voltage & Low $(<25 \mathrm{~V})$ & Moderate $(>50 \mathrm{~V})$ & Low $(<25 \mathrm{~V})$ \\
Linearity & Low & High & High \\
Third order intercept (IP3) & Low $(\sim+28 \mathrm{dBm})$ & High $(\sim+55 \mathrm{dBm})$ & High $(\sim+55 \mathrm{dBm})$ \\
Integration capability & Very good & Good & Good \\
\hline \hline
\end{tabular}




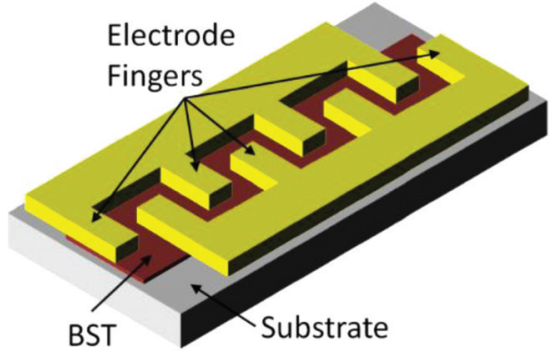

(a)

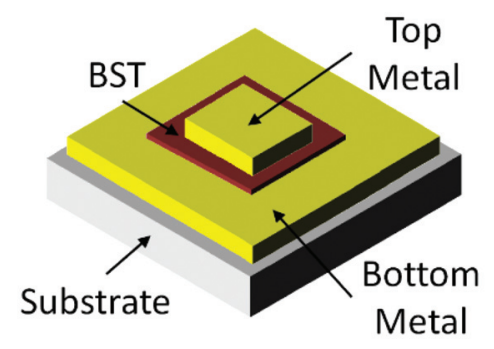

(b)

FIG. 1. Two device designs: (a) coplanar interdigitated capacitor and (b) parallel plate capacitor design. Figure adapted from Ref. 13 with redrawn art work, available in the public domain.

the electrode finger spacing depends on the resolution that can be achieved in the lithography step, most interdigitated capacitors have electrode finger spacing $>2 \mu \mathrm{m}$. The parallel plate capacitor type varactor is fabricated using two metal layers with the BST thin film sandwiched between the two. Typical BST thin film layer thickness is less than $0.4 \mu \mathrm{m}$. Because of the thickness of BST layer, the parallel plate type has higher electric field compared to the interdigitated type for the same applied DC potential between the electrodes. Large capacitance tunability is achieved using parallel plate varactors $(>60 \%)$ compared to the interdigitated capacitors. Also, parallel plate varactors have higher Q compared to interdigitated capacitors. Best results obtained to date have been varactors that used pulsed laser deposition (PLD) for processing of the BST thin films. ${ }^{14}$ Fig. 2 shows the dielectric properties of the BST thin film obtained on a 4 in. diameter R-cut sapphire substrate. The dielectric/capacitance tunability is better than $4: 1$ and the dielectric loss-tangent is below 0.01 at $10 \mathrm{GHz}{ }^{14}$

\section{Integrated varactor devices}

Figure 3 shows a photograph of a BST thin film varactor shunted to ground showing two distinct metal layers with the overlap area of the two metal layers defining the varactor. The BST thin film is sandwiched between the two metal layers. This integrated varactor is implemented in shunt configuration (variable capacitor connected to ground) with coplanar transmission lines (denoted Ground, Signal, Ground) at the input and output. The device is called the varactor shunt switch, as it behaves like a voltage controlled switch for allowing a RF signal to pass or not pass through. ${ }^{5}$ Since

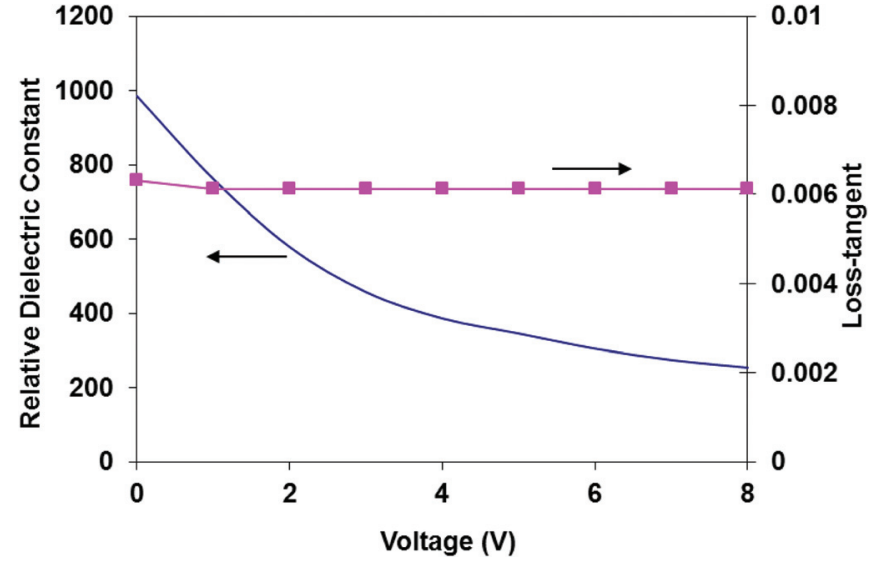

FIG. 2. The dielectric properties of the $0.25 \mu \mathrm{m}$ thick BST thin film on a 4 in. R-cut sapphire substrate. The dielectric properties were obtained by matching measured scattering parameters of a varactor device to an electrical model over a wide frequency range. The dielectric loss-tangent shown is calculated at $10 \mathrm{GHz}$. Reproduced by permission from Subramanyam et al., IEEE Trans. Ultrason. Ferroelectr. Freq. Control 57, 1692 (2010). Copyright 2010 by IEEE.

the BST film has high relative dielectric constant at $0 \mathrm{~V}$ (and hence the shunt capacitor providing a high capacitance to ground), the RF signal is shunted to ground and reflected back resulting in the OFF state of the device. Applying a DC voltage to the signal line reduces the dielectric constant of the film to a lower value (and lower capacitance) allows most of the RF signal to go to the output port resulting in the ON state of the device.

This device has been used as a characterization tool for BST and other dielectric thin films, including polymeric thin films due to its simple nature. The equivalent electrical circuit model for the varactor shunt switch is shown in Figure 4.

In the electrical equivalent circuit, the $\mathrm{C}(\mathrm{V})$ represents the voltage dependent capacitance of the varactor. $R(V)$ is the shunt resistance of the varactor, modeling the leakage through the BST layer, $\mathrm{R}_{\mathrm{s}}$ is the parasitic series resistance of the varactor due to metal electrodes, and $\mathrm{L}$ is the parasitic inductance associated with the varactor, again due to the metal electrodes in the device. Each of these can be precisely

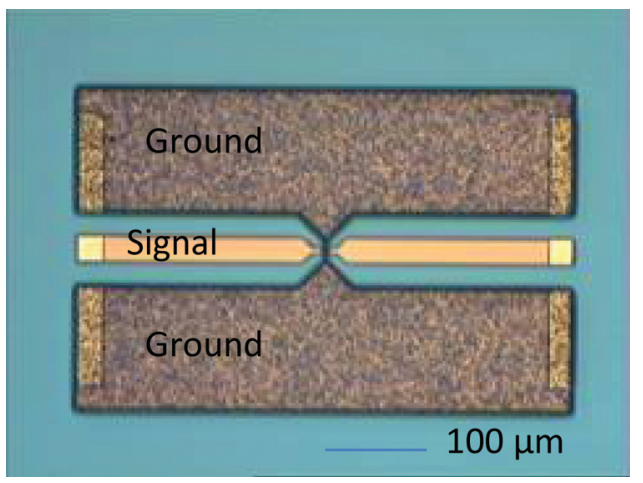

FIG. 3. BST thin film based varactor shunt switch. Such devices have been used for characterization of BST thin films, as well as applications such as switching, phase shifters, and agile antennas. Reproduced by permission from Subramanyam et al., IEEE Microw. Wirel. Compon. Lett. 15, 739 (2005). Copyright 2005 by IEEE. 


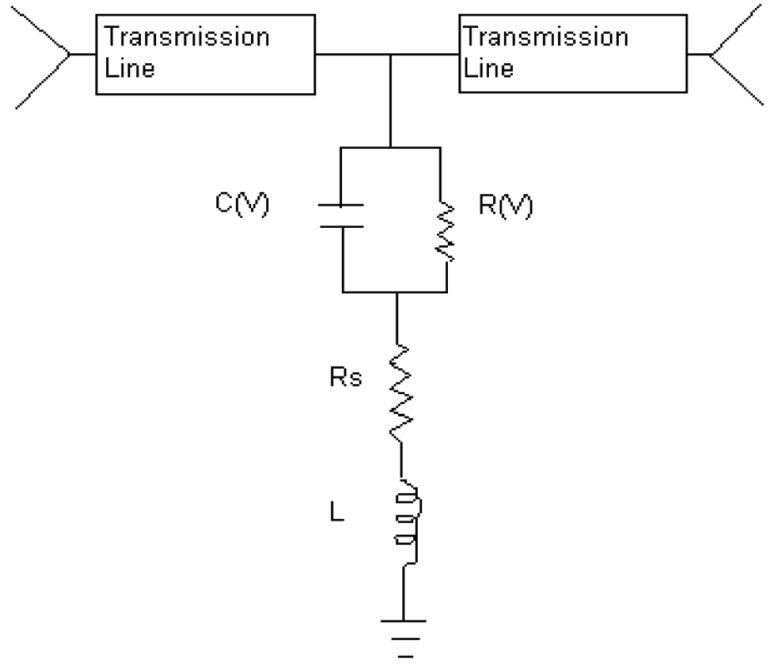

FIG. 4. Equivalent electrical circuit for a shunt varactor device (varactor shunted to ground). Reproduced by permission from Subramanyam et al., IEEE Microw. Wirel. Compon. Lett. 15, 739 (2005). Copyright 2005 by IEEE.

calculated based on the geometry of the parallel plate varactor as follows:

$$
\mathrm{C}(\mathrm{V})=\varepsilon_{\mathrm{r}} \cdot \varepsilon_{0} \cdot \mathrm{A} / \mathrm{d}
$$

where $\varepsilon_{\mathrm{r}}$ is the relative dielectric permittivity of BST layer, $\varepsilon_{0}$ is the dielectric permittivity of free space, $\mathrm{A}$ is the area of overlap of the top and bottom electrodes, and d is the BST layer thickness

$$
\mathrm{R}(\mathrm{V})=1 /(\omega \mathrm{C} \cdot \tan \delta),
$$

where $\omega$ is the angular frequency in radians, $\mathrm{C}$ is the varactor capacitance, and $\tan \delta$ is the dielectric loss-tangent of the BST layer. $\mathrm{R}(\mathrm{V})$ is typically very high $(>10 \mathrm{M} \Omega)$ at low frequencies and decreases at higher $\mathrm{GHz}$ frequencies

$$
\mathrm{R}_{\mathrm{s}}=1 /(\sigma . \mathrm{w} . \mathrm{t})
$$

where 1 is the length of the bottom electrode metal layer, $\sigma$ is the conductivity of the bottom metal layer, $w$ is the width of the electrode layer, and $t$ is the thickness of the bottom metal layer.

The parasitic inductance $\mathrm{L}$ is typically negligible, less than $0.01 \mathrm{nH}$ in most parallel plate varactor devices. L increases for interdigitated varactor devices. For interdigitated varactor devices, it is important to keep the length of the fingers small so that the parasitic inductance is kept to a minimum.

To characterize the thin films of BST, the swept frequency scattering parameters are obtained for different applied voltages using a $\mathrm{RF} / \mathrm{microwave}$ probe station and a vector network analyzer (VNA). The data files are imported to AWR software tool, in which the schematic electrical equivalent circuit for the varactor device is drawn, simulated, and the S-parameters of the electrical circuit are matched to the S-parameters to the measured data. An example of a matched simulated and measured data files are shown in Figure 5 for a $5 \times 5 \mu \mathrm{m}^{2} \mathrm{BST}$ thin film varactor at $0 \mathrm{~V}$. The $\mathrm{S} 21(\mathrm{~dB})$ stands for the ratio of the output power to the input

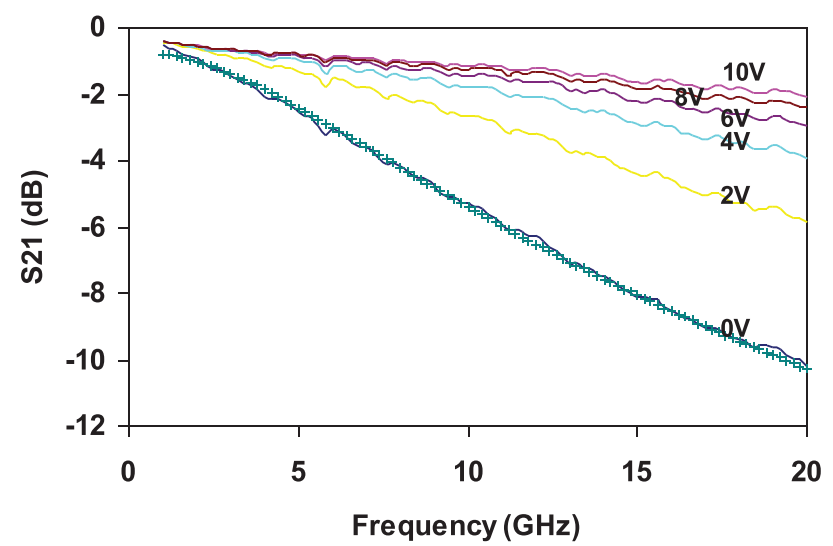

FIG. 5. Measured swept frequency S21 (ratio of output power to the input power in $\mathrm{dB}$ ) for a $5 \times 5 \mu \mathrm{m}^{2}$ BST thin film varactor for various dc bias voltages. The measured data are matched to the modeled data for $0 \mathrm{~V}$ dc bias.

power. The S21 is the lowest at $0 \mathrm{~V}$ and improved with applied DC bias.

From the electrical model, one can obtain the relative dielectric constant and the loss-tangent of the BST thin film for different dc bias voltages, once we have the thickness of the BST thin film, and the values for the $\mathrm{C}(\mathrm{V}), \mathrm{R}(\mathrm{V}), \mathrm{R}_{\mathrm{s}}$, and L. Figure 2 is an example of the voltage dependent dielectric properties obtained using this procedure.

The quality factor (Q) of the capacitor can also be obtained from the electrical equivalent circuit as

$\mathrm{Q}=$ capacitive reactance/equivalent series resistance (ESR) at a desired frequency.

Quality factor can also be obtained approximately as $1 / \tan \delta$, where $\tan \delta$ is the loss-tangent of the dielectric thin film. Another figure of merit of interest for varactors is the commutation quality factor $(\mathrm{CQF})^{15}$

$$
\mathrm{CQF}=(\mathrm{n}-1)^{2} /\left(\mathrm{n} \cdot \tan \delta_{1} \cdot \tan \delta_{2}\right),
$$

where $\mathrm{n}$ is the varactor tunability ratio, defined as $\mathrm{C}(0) / \mathrm{C}(\mathrm{V})$, $\tan \delta_{1}$ is the loss-tangent at $0 \mathrm{~V}$, and $\tan \delta_{2}$ is the loss-tangent at the bias voltage V. For the state of the art BST varactors on sapphire and high resistivity $\mathrm{Si}$, the $\mathrm{Q}$ is higher than 100 at $1 \mathrm{GHz} .{ }^{14}$ For a typical $5 \times 5 \mu \mathrm{m}^{2}$ varactor, conservative values of $\mathrm{n}=3, \tan \delta_{1}=0.02$, and $\tan \delta_{2}=0.01$ gives a $\mathrm{CQF}$ value of 6650 . CQF of greater than 5000 is required for $\mathrm{RF} /$ microwave applications. The current oxide thin film varactor technology can easily achieve this requirement.

\section{Multiferroic thin film devices}

Multiferroic composite materials consisting both a magnetic phase and a ferroelectric phase are of great interest, as they offer the possibility of magneto electric (ME) coupling, i.e., electric field manipulation of magnetic properties (converse ME effect) or vice versa (direct ME effect), ${ }^{16-19}$ and have led to many novel multiferroic devices. ${ }^{20-26}$ One important series of such multiferroic devices is the electrostatically tunable microwave multiferroic signal processing devices, including tunable resonators, ${ }^{24}$ phase shifters, ${ }^{25}$ and tunable filters. ${ }^{27}$ Compared to conventional tunable microwave magnetic devices that are tuned by magnetic field, 
these electrostatically tunable microwave multiferroic devices are much more energy efficient, less noisy, compact, and light-weight. The magneto electric effect can be realized in multiferroic composites through a strain/stress mediated interaction ${ }^{15-29}$ which enables effective energy transfer between electric and magnetic fields and leads to important new functionalities and new devices. A key component for reconfigurable $\mathrm{RF} /$ microwave electronics is a voltage tunable inductor. Tunable inductors have been demonstrated using a multiferroic thin film deposited on a piezoelectric substrate, up to low GHz frequencies. ${ }^{23}$ A voltage applied to the piezoelectric thin film substrate physically deforms the conductor and tunes the self-inductance of the conductor. Multiferroic device research is still in its infancy compared to oxide thin film varactor technology. Table II summarizes the technology areas that voltage tunable oxides and multiferroics can make a significant impact. The table also includes key references that have demonstrated RF/microwave applications.

\section{ADVANCES IN GROWTH-PROCESS SCIENCE METHODS}

There are a variety of techniques for growing/fabricating complex oxide (BST and other oxides) thin films, namely, PLD, RF magnetron sputtering, molecular-beam epitaxy (MBE), chemical solution deposition (CSD), and metal organic chemical vapor deposition (MOCVD). For the purpose of this discussion, CSD includes all liquid based deposition techniques including sol-gel and metal organic solution deposition (MOSD) processes. Although very promising research results have spawned from utilizing each of these growth methods, it must be noted that most of the literature based demonstrations of complex oxide (BST-based) thin films have employed growth techniques characterized by small area deposition, high cost, and/or low-throughput. These research techniques have (and will continue to) add significantly to the knowledge base of complex oxide film properties and the relationships between processing parameters and film properties. However, other deposition technologies will be required for implementation of BST-based devices in large volume, for both military and commercial applications. Selecting the most appropriate technique for a particular situation will depend on many factors, including the requirements of the device, the requirements of the substrate, and the economics of ultimate implementation in the final application. Table III presents a summary of the attributes and disadvantages of each of the film growth techniques considered in this discussion.

\section{A. PLD}

PLD is a thin film growth technique whereby a high power pulsed laser beam is focused on a target (the target material which is evaporated by the laser is normally mounted on a rotating disc attached to a support) of the material (in a vacuum chamber) that is to be deposited onto a substrate (Figure 6).

The material is vaporized from the target (laser plasma plume) which deposits it as a thin film on the desired substrate. Due to its ease of operation, rapid deposition time,

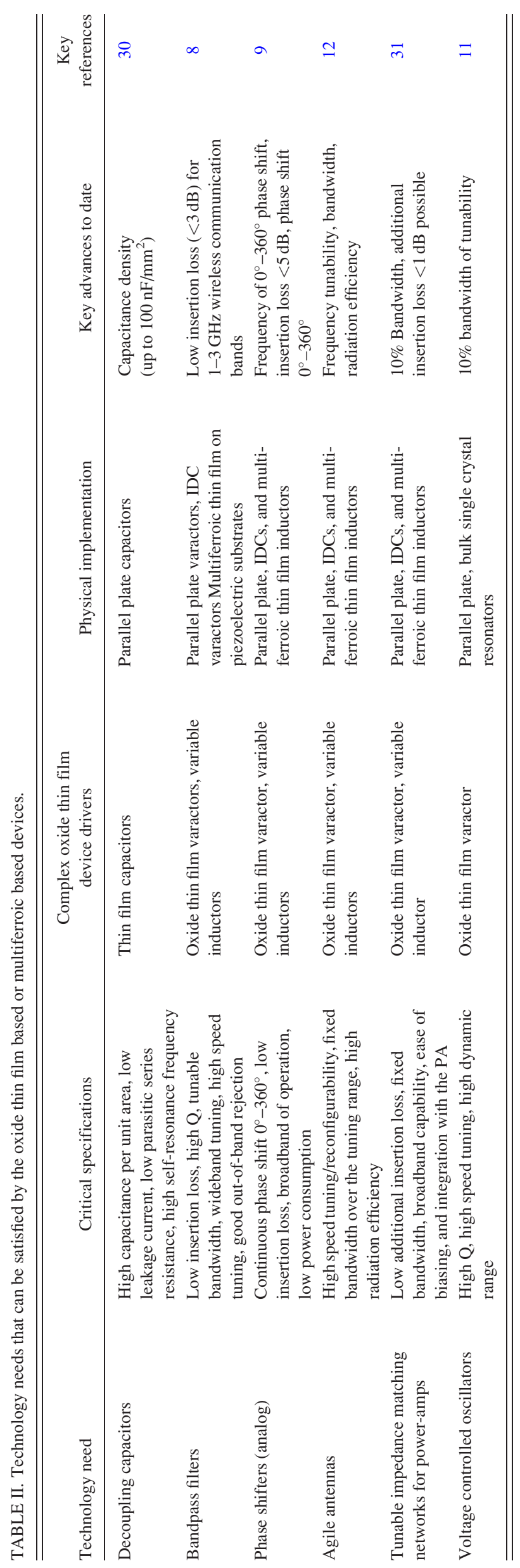


TABLE III. Summary of the attributes and shortfalls of the various complex oxide thin film growth techniques.

\begin{tabular}{|c|c|c|c|c|c|}
\hline \multirow[b]{2}{*}{ Method attributes } & \multicolumn{5}{|c|}{ Complex oxide film growth techniques } \\
\hline & PLD & RF sputtering & MBE & CSD & MOCVD \\
\hline Industry standard & No & Yes & Yes & Yes & Yes \\
\hline Large area ( $>4$ in. diameter) & No & Yes & Yes & Yes & Yes \\
\hline Throughput & Moderate & High & Low & High & High \\
\hline Deposition rate & High & High (low for off axis) & Low & High & High \\
\hline Equipment cost & Moderate & Moderate & High & Low & Moderate \\
\hline Compositional control & Excellent & Moderate & Excellent & Excellent & Excellent \\
\hline $\begin{array}{l}\text { Large area compositional } \\
\text { uniformity (4-in. wafer) }\end{array}$ & Fair $^{\mathrm{a}}$ & Fairly good & Good & Good & Excellent \\
\hline $\begin{array}{l}\text { Large area thickness } \\
\text { uniformity (4-in. wafer) }\end{array}$ & Fair $(<7 \%)$ & Moderate & Good & Good & Good \\
\hline Microstructure & $\begin{array}{l}\text { Highly oriented to } \\
\text { epitaxial }\end{array}$ & Highly oriented/textured & $\begin{array}{l}\text { Highly oriented, epitaxial, } \\
\text { single crystal }\end{array}$ & $\begin{array}{l}\text { Polycrystalline to highly } \\
\text { oriented/textured }\end{array}$ & $\begin{array}{l}\text { Highly oriented } \\
\text { to epitaxial }\end{array}$ \\
\hline Large area uniformity & Fair & Good & Good & Good & Good \\
\hline $\begin{array}{l}\text { Surface morphology } \\
\text { (surface smoothness) }\end{array}$ & Moderate & Moderate smoothness & $\begin{array}{l}\text { Excellent (very } \\
\text { smooth surfaces) }\end{array}$ & $\begin{array}{l}\text { Excellent (very } \\
\text { smooth surfaces) }\end{array}$ & $\begin{array}{c}\text { Good (fairly } \\
\text { smooth surfaces) }\end{array}$ \\
\hline Film adhesion & Excellent & Excellent & Excellent & Good & Good \\
\hline Conformal deposition & No & No & No & Yes & Yes \\
\hline Processing temperatures & High-moderate & High-moderate & High-moderate & Moderate to low & Moderate \\
\hline
\end{tabular}

${ }^{\mathrm{a}}$ With beam profile control.

and excellent target-film stoichiometry transfer, PLD is by far the most popular growth method for complex oxides/BST thin films. ${ }^{32-35}$ The PLD method is excellent for depositing high quality crystalline films, epitaxial films, multi-layer and various superlattices with high deposition rates. ${ }^{36,37}$ The development of new laser technology, such as lasers with high repetition rate and short pulse durations, have made PLD a very competitive tool for the growth of thin, highly crystalline films with complex stoichiometry. Factors which influence film deposition that must be optimized to grow high quality complex oxide thin films include composition of the target material, pulse energy of laser, distance from target to substrate, and the type of gas and pressure in the chamber (oxygen, argon, etc.). Although the PLD possesses many quality film growth attributes, the length scale of the film thickness uniformity is limited (typically $\sim 1$ in. $^{2}$ ), and the

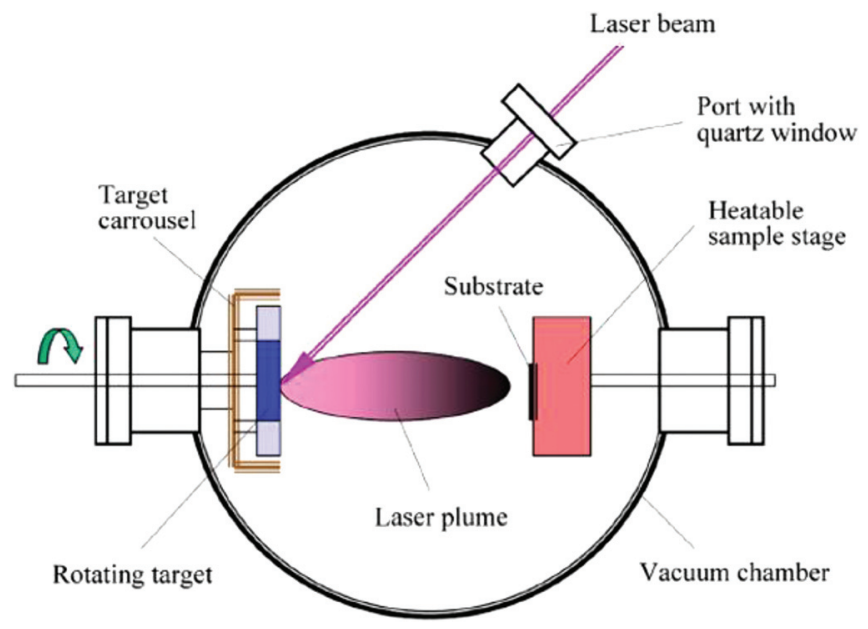

FIG. 6. Schematic diagram of a typical PLD system (Reprinted from source: http://www.andor.com/Portals/0/Pulsed\%20Laser\%20Deposition.jpg). process inherently creates macro particles/particulate debris on the film surface. In addition, the PLD technique is not standard to the semiconductor industry and is not part of a typical semiconductor foundry fabrication line. These drawbacks make PLD less suited to meeting the surface flatness and large area uniformity criteria required for complex oxide tunable device performance and scale-up. Recent advances by companies, such as Neocera and SolMateS,${ }^{38}$ have developed PLD tools which are capable of large area film deposition of complex oxides over 6 in. or 8 in. diameter wafers with minimum droplet/particles. These systems are targeting the MEMS market, and the PLD tools offer high-yield piezo performance and flexibility primarily for customized lead zirconate titanate (PZT) compositions. At present, SolMateS has not qualified other complex oxide films (non PZT-based films) via this method. Also, Varanasi et al. showed that process optimization for large area PLD can produce highly tunable uniform films over 4 in. diameter wafers utilizing the Neocera Pioneer 180 PLD system. ${ }^{39}$ Although no semiconductor foundries possess such large area PLD growth tools in-their manufacturing lines, the development and increased sales of such tools holds significant promise for these large area PLD tools to enter into foundry manufacturing lines in the future. For now, PLD is an excellent lab-scale research tool for screening new and novel complex oxide film compositions and material designs.

\section{B. RF sputtering deposition}

In simplest terms, sputter deposition is a physical vapor deposition (PVD) technique which "sputters" or "removes" material from a target source and deposits this ejected material onto a substrate support or wafer (Figure 7).

rf magnetron sputter deposition of complex oxide films, such as BST, using a multicomponent BST oxide target, is a 


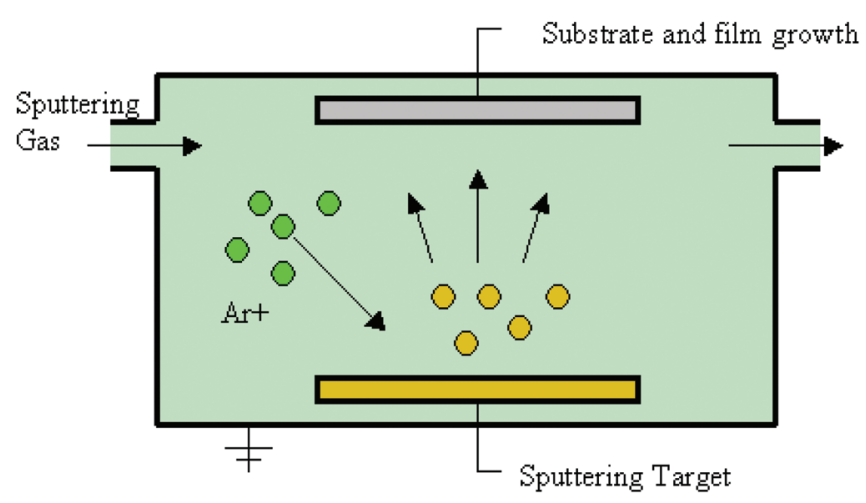

FIG. 7. Schematic diagram of a typical sputtering system. Diagram depicts a "sputter-up" configuration.

widely used deposition/growth method to produce highquality complex oxide thin films. RF sputtering, like most PVD techniques, results in highly textured/oriented thin films (Figure 8). A special configuration of the rf sputtering techniques, referred to as off-axis rf magnetron sputtering technique, consists of a target and of a substrate placed on a grounded sample holder positioned at $90^{\circ}$ of the target offaxis configuration, yields highly crystalline growth of thin films on single crystal substrates, offering a well-defined orientation which defines epitaxy. Unfortunately, the off-axis sputtering process is hampered by its very low deposition rate.

Although RF sputtering offers large area deposition ( $>4$ in. wafer) and is an industry standard deposition tool, however, there are issues with controlling the stoichiometry in films. As with all film growth techniques, it is important to highlight that the effect of film processing parameters on the composition and microstructure of the complex oxide films, such as BST, are critical for achieving the optimum material properties. Specifically, it has been shown by a number of authors that the electrical properties of BST films are quite sensitive to $\mathrm{Ti}$ nonstoichiometry, which is conventionally denoted by the $(\mathrm{Ba}+\mathrm{Sr}) / \mathrm{Ti}$ ratio. It is typically observed that sputtering from a single multicomponent stoichiometric oxide target produces a nonstoichiometric oxide thin film. To avoid this problem, researchers have deposited/grown films under high gas pressure, employed target-substrate off-axis geometries, and have used compositionally adjusted targets. Compositionally controlled rf sputtered BST films have been demonstrated using a stoichiometric $\mathrm{Ba}_{0.5} \mathrm{Sr}_{0.5} \mathrm{TiO}_{3}$ target by control of the background processing gas pressure. Specifically, Im et al. ${ }^{40}$ determined that the $(\mathrm{Ba}+\mathrm{Sr}) / \mathrm{Ti}$ ratios of these BST films could be adjusted from 0.73 to 0.98 by changing the total $\left(\mathrm{Ar}+\mathrm{O}_{2}\right)$ process pressure, while the $\mathrm{O}_{2} / \mathrm{Ar}$ ratio did not strongly affect the metal ion composition. It was determined that there was significant interplay between the total process pressure, dielectric response, and film structure and surface quality. It was found that $(\mathrm{Ba}+\mathrm{Sr}) / \mathrm{Ti}$ ratios of 0.9 or higher, exhibited a polycrystalline microstructure, and possessed root-mean-square/rms roughness on the order of $3 \mathrm{~nm}$. In contrast, films with $(\mathrm{Ba}+\mathrm{Sr}) / \mathrm{Ti}$ ratios below 0.85 , the BST became nanocrystalline with a much smoother surface morphology exhibiting a rms roughness $<2 \mathrm{~nm}$. In terms of dielectric response, the near-stoichiometric BST film $(\mathrm{Ba}+\mathrm{Sr}) / \mathrm{Ti}$ ratios of 0.95 displayed the largest tunability, $\sim 74 \%$, while the lowest dielectric loss of 0.0047 at zero bias was found for the BST film with a $(\mathrm{Ba}+\mathrm{Sr}) / \mathrm{Ti}$ ratio of 0.73 . Mechanistically, these results are easily explained by the fact that the pressure dependencies of the individual fluxes of $\mathrm{Ba}$, $\mathrm{Sr}$, and Ti impinging on the substrate (i.e., the different constituents have different, pressure dependent angular scattering distributions in the gas phase, due to their different masses). ${ }^{41}$

In general, achieving precise control over film stoichiometry is more complex, compared to the PLD technique; however, it can be accomplished by selecting the proper process pressure. In other words, the deposition process pressure can be experimentally tailored to achieve the desired dielectric response to be optimized. Although, it must be kept in mind that level of stoichiometric control is limited to major constituents of the film, for example, the $\mathrm{Ba}, \mathrm{Sr}$, and Ti in BST films, hence, deposition of undoped BST (3 components) thin films has been fairly successful via sputtering. ${ }^{42-44}$ However, successful fabrication of multicomponent (4 or more elemental components, i.e., doped BST) and designer/compositionally tailored/graded BST based films has not been successfully accomplished with precise control of film stoichiometry. One of the major reasons for this is that the yield and sticking coefficients of the various components are not equal, and therefore, precise control of the film stoichiometry is often difficult. Therefore, the major shortfall of stoichiometry control in rf sputter deposition occurs for dopant-level film constituents, for example, a BST film that is doped $1 \%$ with $\mathrm{Mg}$. In such cases of low

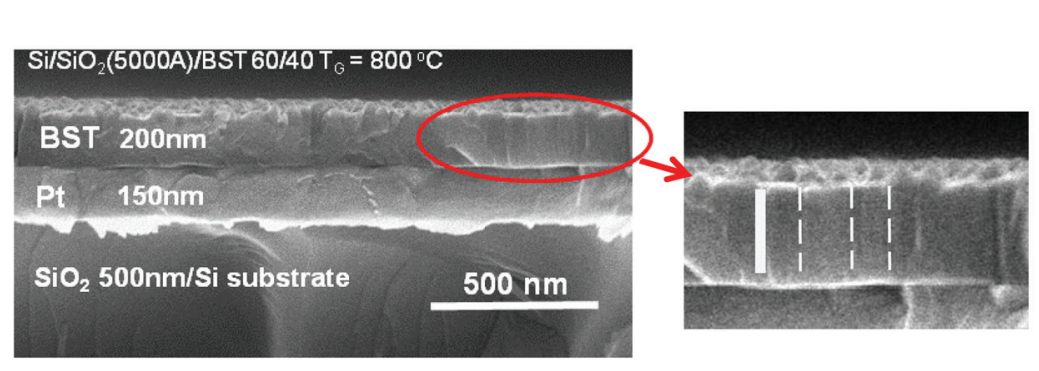

(a) (b)

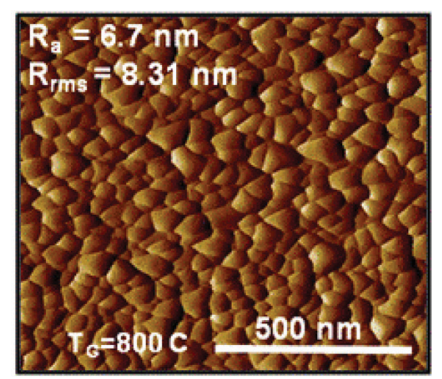

(c)

FIG. 8. BST thin film deposited on Pt bottom electrode on $\mathrm{SiO}_{2} / \mathrm{Si}$-wafer. (a) Cross-section FESEM micrograph, (b) enlargement of BST film showing textured film, and (c) AFM micrograph showing surface morphology of the rf sputtered BST film. Figure courtesy of M. W. Cole's research group, Army Research Laboratory-WMRD, 2012. 


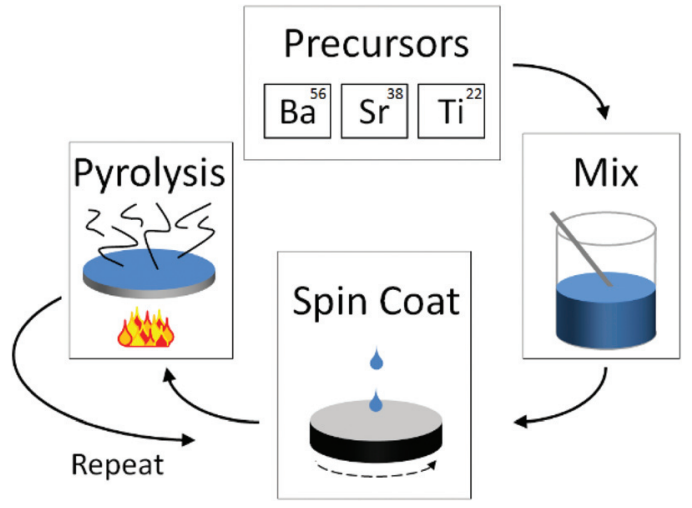

FIG. 9. Schematic diagram of the MOSD spin-coating process for complex oxide (BST) thin film fabrication.

dopant concentrations within a multi-component film, such as BST, rf-sputtering is not the most suitable film deposition technique.

\section{CSD}

CSD was adopted for the fabrication of PZT in the 1980s. ${ }^{45}$ The CSD technique has attracted extensive attention in recent years as a cost-effective technique for largescale fabrication and manufacture of high quality complex oxide films. In general, CSD includes all wet chemical processes involving solution based chemical reactions of precursors during the formation of the films. The term "sol-gel" is often used to refer to a CSD route beyond the original definition of a sol-gel process, which involves hydroxylation and condensation of organometallic precursors containing network formers (e.g., silicon, titanium, etc.). The processing techniques applied in the CSD route can be categorized as spin coating, dip coating, spray coating, stamping (or printing), etc. The MOSD technique is analogous with CDS, whereby alkoxide precursors are employed for growth of complex oxide thin films. The CDS and/or MOSD technique using carboxylate-alkoxide precursors is one of the simplest techniques available to prepare pure and doped stoichiometric multicomponent complex oxide thin films. Among the advantages of the CSD/MOSD processing are low processing temperature, precise composition and thickness control, low equipment cost, high deposition rates, uniform deposition over large area substrates, and it is an industry standard film fabrication technique. ${ }^{46}$ Although CSD/MOSD has numerous attributes, it is often problematic to achieve successful chemical compatibility of the chemical precursors.
Another drawback of this film growth technique is that some of the film precursors require chemical distillation, which adds unwanted complexity and time to the film fabrication process. However, overall, this is an excellent technique to grow films of complex oxides.

A schematic for the MOSD film growth technique and representative complex oxide films fabricated by this technique are displayed in Figures 9 and 10, respectively. ${ }^{47,48}$ In the MOSD process, in addition to the alkoxide/organic metallic precursors required to form to the complex oxide film, various other chemicals, such as acetic acid and 2 methoxyethanol, are employed as solvents and solution viscosity modifiers. The stoichiometric precursor solutions are then concentration optimized and spin/dip coated onto the desired substrates. Subsequent to each layer coating, the films are pyrolyzed (temperatures $\sim 350-500{ }^{\circ} \mathrm{C}$ for 10-30 min) in order to evaporate solvents and organic addenda and form an inorganic amorphous film. The spin/dip coat pyrolization process is repeated until the desired nominal film thickness is achieved. The as-deposited films are amorphous, hence film crystallinity is achieved via a post deposition annealing step. It should be noted that the fabrication of high quality complex oxide films via CSD/MOSD is highly dependent on the preparation of optimized precursor solutions with desired solubility, reactivity, concentration, and viscosity, substrate selection, and buffer layer development. In addition, it is important to optimize the thickness for each coating layer in a CSD/MOSD process to yield non-cracking and non-porous films. It has been demonstrated that a layer-by-layer automated dip-coating process $^{49}$ allows nano-layer engineering and complete organic species burnout, leading to high quality and high density thin films with performance comparable or superior to the ones produced by PLD or sputtering. ${ }^{50-52}$ BST50/50 films biaxially grown on $\mathrm{LaAlO}_{3}$ substrates via this process exhibit a very low loss as shown in Figure 11. The full-width-half-maximum (FWHM) of a rocking curve scan at the (002) plane is as small as $0.06^{\circ} .^{51}$ Shown in Figure 12 is a cross-section SEM image of a $\mathrm{PbTiO}_{3}$ film coated on glass substrate via the layer-by-layer dip coating process, indicating a very dense film.

In addition to thin film growth, thick films $(>10 \mu \mathrm{m})$ have also been demonstrated via spin coating ${ }^{53}$ and printing ${ }^{54}$ techniques with porosity levels acceptable for certain applications. It is also worth mentioning that Abe et al. ${ }^{55}$ have succeeded in low temperature fabrication of polycrystalline films of spinel structure $\left((\mathrm{M}, \mathrm{Fe})_{3} \mathrm{O}_{4}, \mathrm{M}=\mathrm{Fe}, \mathrm{Co}, \mathrm{Ni}, \mathrm{Zn}, \mathrm{Mn}\right.$, etc. $)$ via a spin spray plating (SSP) process. Nonetheless, no work

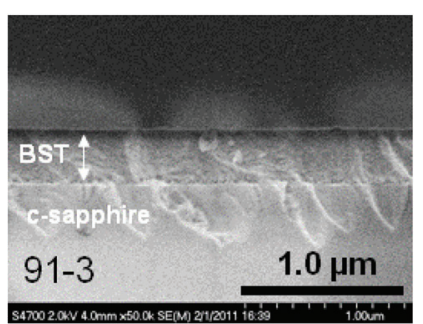

As-Grown

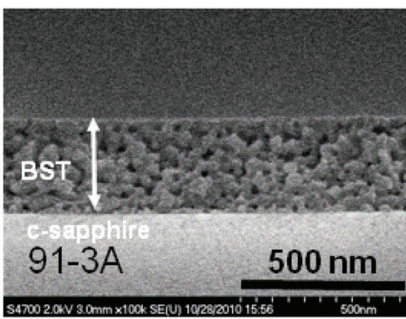

Annealed

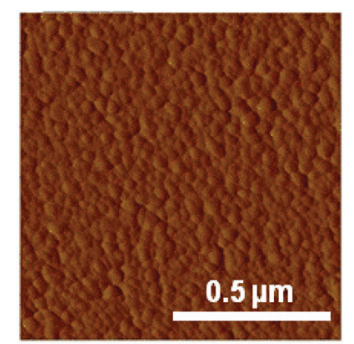

$1 \mu \mathrm{m} \times 1 \mu \mathrm{m} R_{\mathrm{RMS}}=2.67 \mathrm{~nm}$
FIG. 10. Cross-sectional FESEM micrographs of a BST thin film grown by the MOSD technique, (a) the asgrown film, (b) the post deposition annealed film, and (c) AFM image showing the smooth uniform surface morphology of the crystallized BST film. Figure courtesy of M. W. Cole's research group, Army Research Laboratory 2012. 


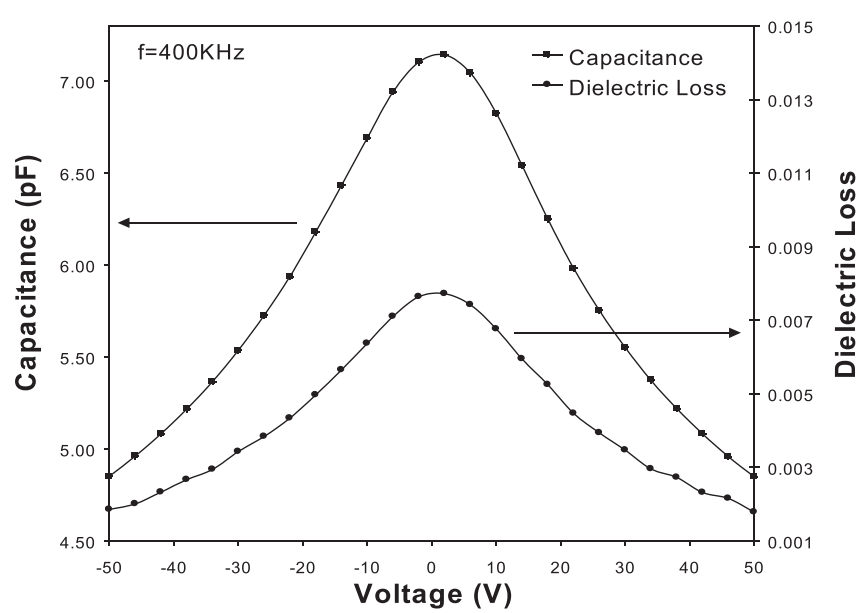

FIG. 11. Capacitance and dielectric loss of a $\mathrm{Ba}_{0.5} \mathrm{Sr}_{0.5} \mathrm{TiO}_{3}$ film grown on a $\mathrm{LaAlO}_{3}$ substrate via CSD. Reproduced by permission from Jiang et al., Integr. Ferroelectr. 28, 68 (2000). Copyright 2000 by Taylor \& Francis.

has been reported on other complex oxide systems by the SSP technique.

\section{MBE}

$\mathrm{MBE}$ is an industry standard tool for the growth of thin films. This technique offers excellent stoichiometric compositional control and the ability to grow compositionally graded dielectric thin films. The major advantages of the MBE growth technique include: (i) Outstanding microstructural control whereby the microstructure of the deposited film is closely controlled, ranging from dense, epitaxial highly oriented, and single crystal films ${ }^{56}$ (ii) high degree of composition control whereby the film properties are easily adjustable to allow for great versatility in depositing a wide variety of complex, multi-component compounds with precisely targeted stoichiometries; (iii) the MBE growth process allows deposition on a wide range of substrate materials and offers ability for large area ( $>4$ in. diameter) wafer coverage; (iv) MBE growth tools are suitable for continuous production whereby MBE production systems have demonstrated operation without failure around the clock for extended time periods; (v) MBE offers growth of complex multi-layered and

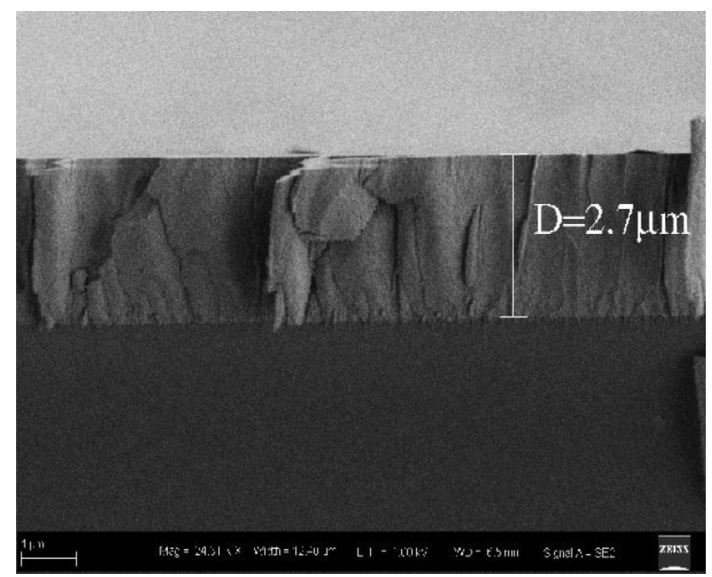

FIG. 12. Cross-section SEM image of a $\mathrm{PbTiO}_{3}$ film coated on glass substrate, showing a very dense film formed via a layer-by-layer CSD route. functionally compositionally graded material structures; and finally (vi) MBE offers extreme film uniformity with precise control of coverage area.

Low energetic, high-purity techniques, such as MBE, have the potential to produce epitaxial complex oxide films with low intrinsic defect concentrations and atomic-layer control. ${ }^{56}$ Oxide MBE growth of cation and oxygen stoichiometric perovskite titanate films has, however, proved challenging due to the fact that complex oxides, such as $\mathrm{SrTiO}_{3}$, do not have a wide "MBE growth window" in which the cation stoichiometry is self-regulating. Low growth rates severely limit practically achievable film thicknesses needed for films in device structures. A popular variant of MBE is the use of volatile metal organic source materials; this is called metal organic MBE (MOMBE) and it is being applied to an increasing variety of complex oxides. ${ }^{5,58}$ For example, the Stemmer research group at UCSB is using MOMBE for the growth of $\mathrm{SrTiO}_{3}$ films that combines an effusion cell for $\mathrm{Sr}$, a plasma source for oxygen, and a metal-organic source (titanium tetra isopropoxide) for $\mathrm{Ti}^{59}$ Advantages of the metal-organic source include a vapor pressure that is orders of magnitude higher than solid Ti. ${ }^{60}$ The MOMBE growth approach has enabled the growth of nearly stoichiometric insulating $\mathrm{SrTiO}_{3}$ films with scalable growth rates and excellent structural quality, as evidenced by the very narrow rocking curves and nearly ideal surfaces with root mean square surface roughness values of less than 0.1 .

The ability to produce thin films with uniform material properties renders MBE desirable for complex oxide device fabrication. Although MBE is an industry standard film deposition tool used in commercial semiconductor foundries, the growth rate of complex oxides via $\mathrm{MBE}$ is very slow and expensive. Recently, BST films fabricated via MOMBE technique achieved device quality dielectric properties. ${ }^{61}$ The MBE technique is also limited by low throughput/yield and is an expensive technique to use and sustain. However, if the cost of this technique becomes commensurate with that of CVD and PVD growth techniques, properly optimized MBE grown complex oxide thin films will become widespread and mainstream.

\section{E. MOCVD}

CVD is a process in which gaseous source materials are chemically reacted to produce films and coatings. MOCVD is a variation of CVD, for which the precursors are compounds of metal atoms and organic ligands. MOCVD of complex oxides is usually (but not always) accomplished at reduced pressure. Similar to MBE, MOCVD reactions are driven solely by thermal energy. Each atom arrives at the surface of the growing film with no kinetic energy, enabling very high quality films. Similar to MOSD, MOCVD can produce conformal coatings on three dimensional substrates and over device topography. This was the main incentive to investigate both MOSD and MOCVD to deposit high dielectric constant BST films for DRAM applications, in order to build high density, non-planar capacitor geometries. ${ }^{62,63}$ Similar to other vacuum techniques, MOCVD requires a large up-front capital investment for the equipment. 
However, when implemented in high volume production, MOCVD can provide low overall cost-of-ownership, due to its high throughput and high source material utilization.

For small substrates, MOCVD of complex oxides can be accomplished in a variety of reactor geometries. Scaling these processes up to large wafers and high volume production requires MOCVD reactors designed specifically to provide uniform gas distribution across the substrate. Uniform precursor distribution prevents gas depletion effects, which can cause variation of film thickness and composition across the wafer. Uniform wafer temperature is also important for uniformity of film thickness, composition, and properties. Commercial MOCVD reactors for high volume production of complex oxide films follow designs developed for compound semiconductor films. The reactor chamber is typically a cold-wall or warm-wall vertical cylinder, with an upper gas showerhead and a large horizontal platter for single or multiple wafer loads. Two basic strategies are used to achieve uniform gas distribution; planetary reactors and high speed rotating disk reactors. Planetary reactors use complex wafer motion to ensure that each section of the wafer sees a similar thermal and gas flow history. In high speed rotating disk reactors, the source gases are drawn down to the disk surface by viscous drag, then pushed outward, creating a very thin boundary layer and eliminating recirculation. The high speed rotating disk design has been demonstrated analytically and experimentally to provide excellent uniformity. ${ }^{64,65}$ Both reactor designs are theoretically scalable to any size wafer platter. Uniform deposition of BST films has been demonstrated in both planetary ${ }^{66}$ and high speed rotating disk MOCVD reactors. ${ }^{67}$

MOCVD processes for complex oxide films are highly dependent on the precursor chemicals. The most commonly used precursors for $\mathrm{BST}$ are $\mathrm{Ba}(\text { thd })_{2}, \mathrm{Sr}(\text { thd })_{2}$ and $\mathrm{Ti}(\mathrm{i}-\mathrm{OPr})_{2}(\text { thd })_{2}$. In these designations, "thd" $=2,2,6,6$-tetramethyl-3,5-heptanedionato and "i-OPr" = isopropoxide. These precursors have the metal species bonded to oxygen. However, an oxygen bearing gas is usually added to the MOCVD process, to ensure complete oxidation of the film and removal of organics. The typical BST precursors are solids at room temperature, and require heating to achieve sufficient vapor pressure. The Group IIa precursors require temperatures around $200^{\circ} \mathrm{C}$ to $240^{\circ} \mathrm{C}$ to volatize. At these temperatures, the precursors are near their decomposition point. If these precursors are used in conventional bubbler geometry, and held at high temperature for extended time, their vaporization behavior can change or "age." This aging effect is due to chemical reactions such as decomposition or polymerization of the precursor. Adducts of the Group IIa precursors have been developed, to improve their stability and temperature resistance. The common adducts include tetraethylene glycol dimethyl ether (tetraglyme), pentamethyldiethylenetriamine (pmdeta), and other supplier specific compounds.

Another strategy for MOCVD of complex oxide films is to dissolve the precursors in a suitable solvent and inject the solution into a heated pre-vaporization chamber, known as a flash evaporator. Figure 13 shows a schematic illustration of a typical MOCVD reactor and direct liquid injection system. Thermal aging effects are minimized, since the precursors are stored at ambient temperature until they are injected into

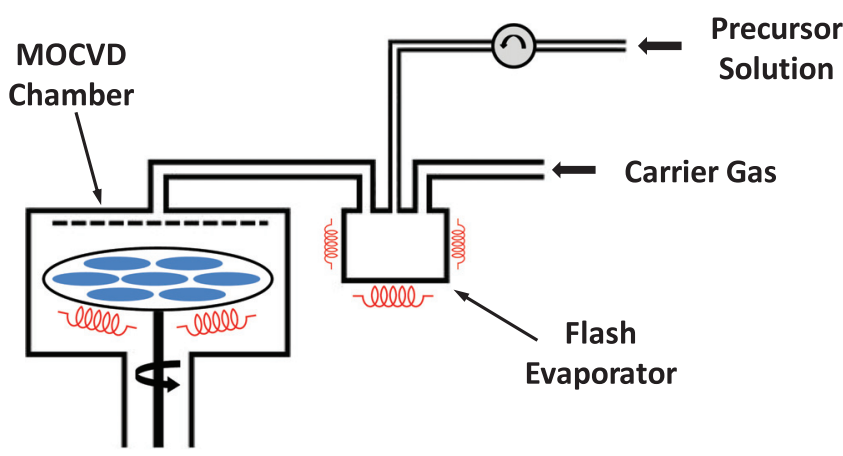

FIG. 13. Schematic illustration of a typical MOCVD reactor with liquid injection system, for deposition of BST and other complex oxide films.

the flash evaporator. This technique requires that the precursor solution is fully vaporized faster than the solution injection rate, in order to maintain consistent composition of the source vapor. All surfaces in the vapor transport path after the flash evaporator must be maintained at or above the evaporator temperature, to avoid precursor loss due to condensation. Cold spots in the vapor transport path can shift film composition through selective loss of low vapor pressure components. Efficient and reliable liquid delivery and flash evaporation systems have been developed for commercial MOCVD reactors and demonstrated for deposition of uniform BST films on large wafers. ${ }^{66,68}$

For MOCVD of complex oxides, such as BST, molar incorporation rates for the individual metal species are precursor and process dependent. The typical procedure for initial process development is to deposit several films and determine their actual composition using a technique such as Rutherford Backscattering Spectroscopy (RBS). This calibration requires consistent precursor materials and stable MOCVD process parameters. The composition of BST films has been shown to vary with substrate temperature during MOCVD, particularly with regard to the $(\mathrm{Ba}+\mathrm{Sr}) / \mathrm{Ti}$ ratio. ${ }^{66,68}$ Within these constraints, initial MOCVD process development and subsequent process control can be done reliably and effectively. Once this calibration is established, MOCVD can easily produce compositionally graded films of nearly any profile. The film composition is a linear function of the molar precursor injection rates. MOCVD can also produce compositionally doped films and interface layers with tailored composition. Compositionally graded BST films deposited using liquid injection MOCVD have shown extremely temperature stable dielectric properties, as well as low leakage current and high tunability of dielectric permittivity. ${ }^{69}$

\section{TECHNOLOGY DRIVERS FOR OXIDE AND MULTI-FERROIC THIN FILMS FOR RF/MICROWAVE APPLICATIONS}

Currently, RF front-end applications support various frequency bands. The replacement of fixed frequency band circuits with reconfigurable circuits will save space and cost for communication systems. ${ }^{70}$ Figure 14 shows a typical front-end of a single frequency band RF communication system.

Extending the RF front end to wide-band frequencies necessitates the requirement of a large number of PAs, 


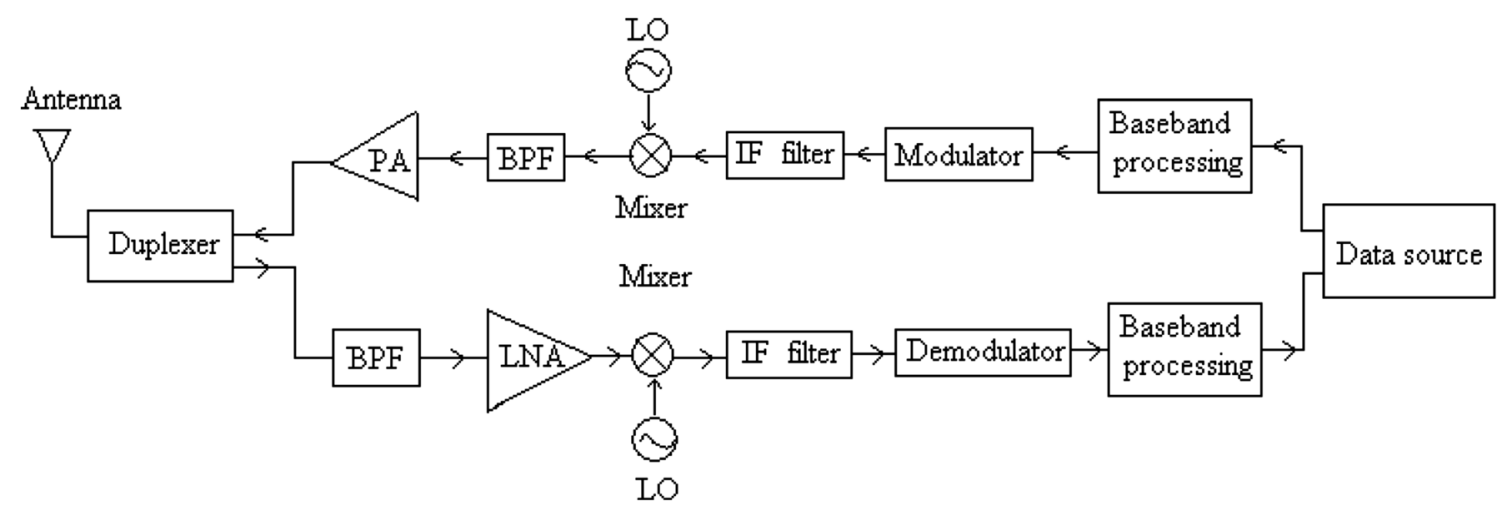

FIG. 14. A typical RF front-end of a single frequency band RF communication system.

filters, duplexers, LNAs, and VCOs. Tunable devices give the opportunity to implement these circuit blocks by optimizing their performance for a given bandwidth by varying either inductance or capacitance or both.

Technology drivers utilizing BST thin film varactors for $\mathrm{RF} /$ microwave applications can be classified into following three categories:

1. Varactors for passive reconfigurable $\mathrm{RF} /$ microwave circuits. In this category, varicaps or varactors are used primarily for reconfiguring passive $\mathrm{RF} /$ microwave circuits such as tunable resonators, band pass filters, band reject filters, and antennas.

2. Adaptive RF electronics. In this category, varactors are integrated with active devices such as impedance matching networks for PAs and VCOs are the main technology drivers.

3. Phase shifters for electronic steering of antenna arrays. In this category, varactors are used primarily for analog (continuous $0^{\circ}-360^{\circ}$ phase shift) or digital (fixed phase shift based on number of bits) phase control over a bandwidth for electronic steering of phased array antennas or digital beam forming applications.

This section describes technologies that can benefit from the use of oxide and multiferroic thin film devices. The technology drivers for thin film varactors include discrete decoupling capacitors for printed circuit boards (PCBs) to passive rf circuit components, impedance matching networks for power amplifiers and low noise amplifiers, voltage controlled oscillators, and analog continuous phase shifters. The technology drivers for thin film multiferroic devices include voltage tunable passive RF components to low frequency magnetic field sensors.

\section{A. Decoupling capacitors}

Noise control is very important for the design of high speed digital integrated circuits (ICs) and PCBs for modern electronic systems. There are several kinds of noise, one of which is the Delta-I noise. ${ }^{71}$ This noise consists of an unwanted voltage on the parasitic inductance power plane connections, caused by the transient currents associated with switching. Assuming a current change $d I$ happens in a time interval of $d t$, it will generate a voltage of $V_{\mathrm{N}}=\mathrm{L}_{\text {eff }} d I / d t$ on the parasitic inductance with an equivalent value of $\mathrm{L}_{\mathrm{eff}}$. Delta-I noise can reduce the driver bias voltage $;^{71}$ in this case, the effect is called the ground bounce. Disturbances of several millivolts to $\sim 1 \mathrm{~V}$ have been reported. These disturbances may jeopardize the operation of sensitive ICs on the PCBs. Delta-I noise waveforms typically consist of two components, an initial peak followed by a resonating waveform. When the drivers switch from one state to another state (logic 0 to logic 1 for example in digital systems), a transient current flows through the connections between the ICs and the power planes. These transients excite a voltage drop on the parasitic inductance of the power/ground connection, thus, resulting in peak noise, which is typically large and occurs for a short duration in comparison to the resonance component of the power planes.

Use of decoupling capacitors is a convenient and efficient way to decouple the power ground noise. By placing a proper capacitor, which has small impedance at the noise frequency, close to each driver, the noise can be effectively filtered. Conventional fixed-value capacitors typically employ dielectrics which have linear polarization to the applied field and have nominally low values of relative dielectric permittivity (e.g., $\varepsilon_{\mathrm{r}}<100$ ). Oxide thin films, such as BST, can be attractive for the decoupling applications due to their high relative dielectric constant.

Decoupling capacitors are normally implemented directly between power bus and ground to reduce the noise in the power bus. While bulk decoupling capacitors are responsible for the decoupling of low frequency noise in a section of a PCB and have larger capacitance $(1-100 \mu \mathrm{F})$, local decoupling capacitors are responsible for the decoupling of higher frequency noise from particular IC and have smaller capacitance $(\mathrm{pF}-\mathrm{nF})$. Multilayer ceramic caps and low inductance chip arrays are commonly used at low frequencies below 50 MHz. High $\varepsilon_{\mathrm{r}}$ oxide thin film capacitors could be useful from $100 \mathrm{MHz}$ to several GHz applications. Figure 15 shows measured impedance of parallel plate BST capacitors of $5 \times 5$ and $20 \times 20 \mu \mathrm{m}^{2}$ area. The slope of the impedance below the resonance frequency depends on the capacitance, the impedance at the resonance frequency (the lowest impedance point) gives the ESR of the capacitor, and the slope above the resonance frequency depends on the effective series inductance (ESL). ${ }^{30}$

For the varactor with an area of $5 \times 5 \mu \mathrm{m}^{2}$, the impedance reduces with the sweeping frequency in all the 


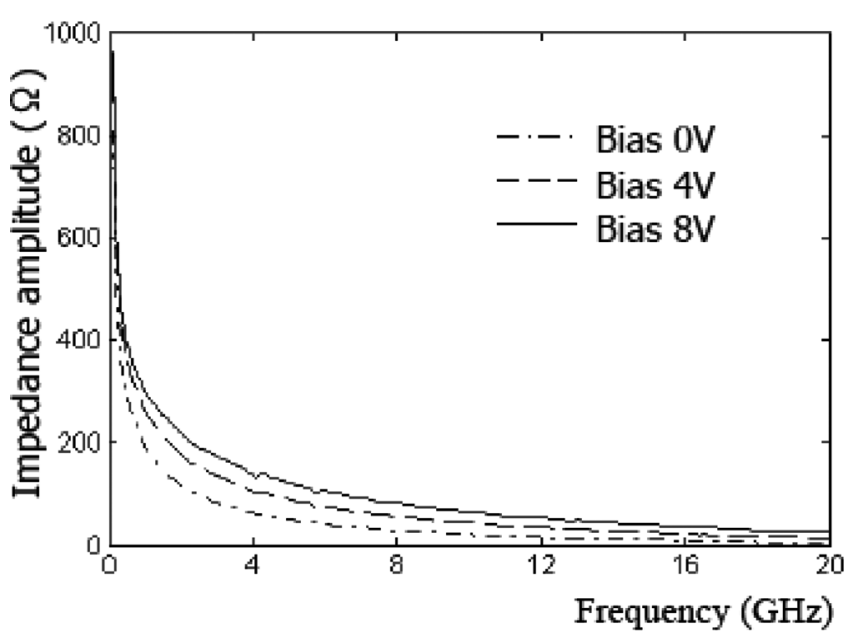

(a) $5 \times 5 \mu \mathrm{m}^{2}$

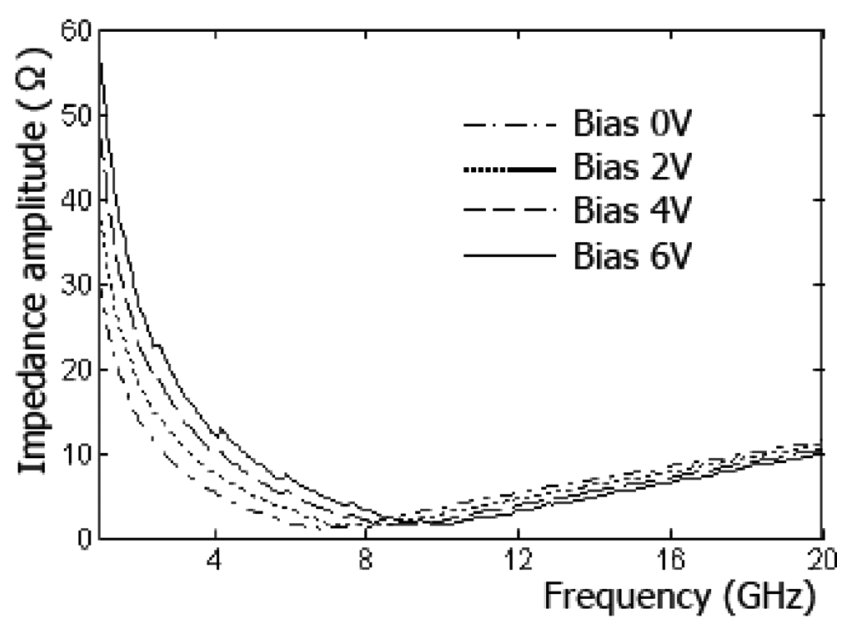

(b) $20 \times 20 \mu \mathrm{m}^{2}$

FIG. 15. Impedance of BST varactors at different bias voltages. Reproduced by permission from H. Li and G. Subramanyam, IEEE Trans. Ultrason. Ferroelectr. Freq. Control 55, 2552 (2008). Copyright 2008 by IEEE.

measured frequency range. Resonance frequency is much higher than the measurement range for this capacitor, since the capacitance is approximately $1 \mathrm{pF}$ at $0 \mathrm{~V}$. For a varactor with an area of $20 \times 20 \mu \mathrm{m}^{2}$, the voltage dependent ESR and self-resonance frequency are extracted as follows: $0 \mathrm{~V}, 1.2 \Omega$ at $6.9 \mathrm{GHz}, 2 \mathrm{~V}, 1.4 \Omega$ at $7.9 \mathrm{GHz}, 4 \mathrm{~V}, 1.6 \Omega$ at $8.8 \mathrm{GHz}$, $6 \mathrm{~V}, 1.8 \Omega$ at $9.8 \mathrm{GHz}^{72}$

As one can see, the resonance frequency is shifted to higher frequencies as the dc voltage reduces the capacitance. The experimental results of the BST varactors confirm that they can be effective decoupling capacitors over a wide frequency range as it has very low ESL $(\sim 25 \mathrm{pH})$. The BST capacitors typically have lower ESR compared to ferroelectric capacitors, such as PZT and $\mathrm{BaTiO}_{3}$, due to their paraelectric phase at room temperature. ${ }^{72}$

Desirable characteristics of decoupling capacitors are: (i) high capacitance density $>1 \mu \mathrm{F} / \mathrm{cm}^{2}$, (ii) dielectric breakdown field $>500 \mathrm{MV} / \mathrm{m}$, (iii) low $\mathrm{ESR}<0.1 \Omega$, and (iv) low ESL $<30 \mathrm{pH}$. Current BST thin film varactors can meet most of the desirable characteristics except the low ESR which is currently in the order of $1 \Omega$ for thin metal electrodes $(\sim 1 \mu \mathrm{m}$ thick). Section $\mathrm{V}$ discusses further on how to improve the ESR. This is a key area of interest for the successful insertion of oxide thin film based capacitors. There is immense potential for oxide thin film based decoupling capacitors.

\section{B. Passive reconfigurable RF/microwave circuits}

\section{Tunable filters}

Filters are one of the most important circuit blocks for modern communication systems. Multi-functional receiver subsystems require broad bandwidth to support multiple bands and still maintain good selectivity in the midst of interfering signals. This has led to an increased demand for preselect filters. Tunable band pass filters offer the opportunity of replacing a bank of fixed bandwidth filters with a single filter to cover a wide frequency range. Traditional band pass filters are realized using combline resonators for $\mathrm{RF} / \mathrm{microwave}$ applications. Tunable band pass filters can be realized monolithically or using discrete components. Table IV compares the different tunable filter technologies. As one can see, BST and other oxide thin film based voltage tunable varactors can meet many of the filter parameters and compete with other technologies well. Most significant advantage of BST thin film varactor based tunable filters is the cost since the thin film varactor technology needs less stringent process steps compared to semiconductor or MEMS based technologies. Most of the research reported in the literature on BST based tunable filters are with BST interdigited capacitors or parallel plate capacitors. Tombak et al. demonstrated the first tunable filters with discrete inductors and tunable interdigited BST capacitors as early as 2003. ${ }^{31}$ An electronically tunable microstrip combline band pass filter using thin film interdigested BST varactors was demonstrated in $2005 .^{8}$ Application of $0-200 \mathrm{~V}$ bias varied the central frequency of the filter from $2.44 \mathrm{GHz}$ to $2.88 \mathrm{GHz}$. Kalkur et al. reported the results of third order combline band pass filters implemented with parallel plate BST discrete capacitors ${ }^{73}$ packaged using a conductive epoxy. These filters can be tuned at low voltages from 0 to $5 \mathrm{~V}$. The schematic of the prototype filter is shown in Figure 16. The measured center frequency of the filter can be tuned from $1.35 \mathrm{GHz}$ to $1.45 \mathrm{GHz}$ by applying a tuning voltage of $3 \mathrm{~V}$. Recently, tunable band pass filter with MOCVD deposited graded BST films to reduce the temperature coefficient of capacitance on sapphire substrate has been demonstrated. ${ }^{74}$

A resonator can also be implemented using a shunt connected capacitor and an inductor to form a tank circuit that exhibits resonance at frequency $f=1 /[2 \pi \sqrt{ }(\mathrm{LC})]$.

\section{Tunable bulk acoustic wave resonators with BST thin films}

Recently, tunable thin film BST and ST acoustic resonators are attracting the attention of many investigators. ${ }^{75-80}$ These resonators have applications as switchable filters in cell phones over surface acoustic wave devices because of their 

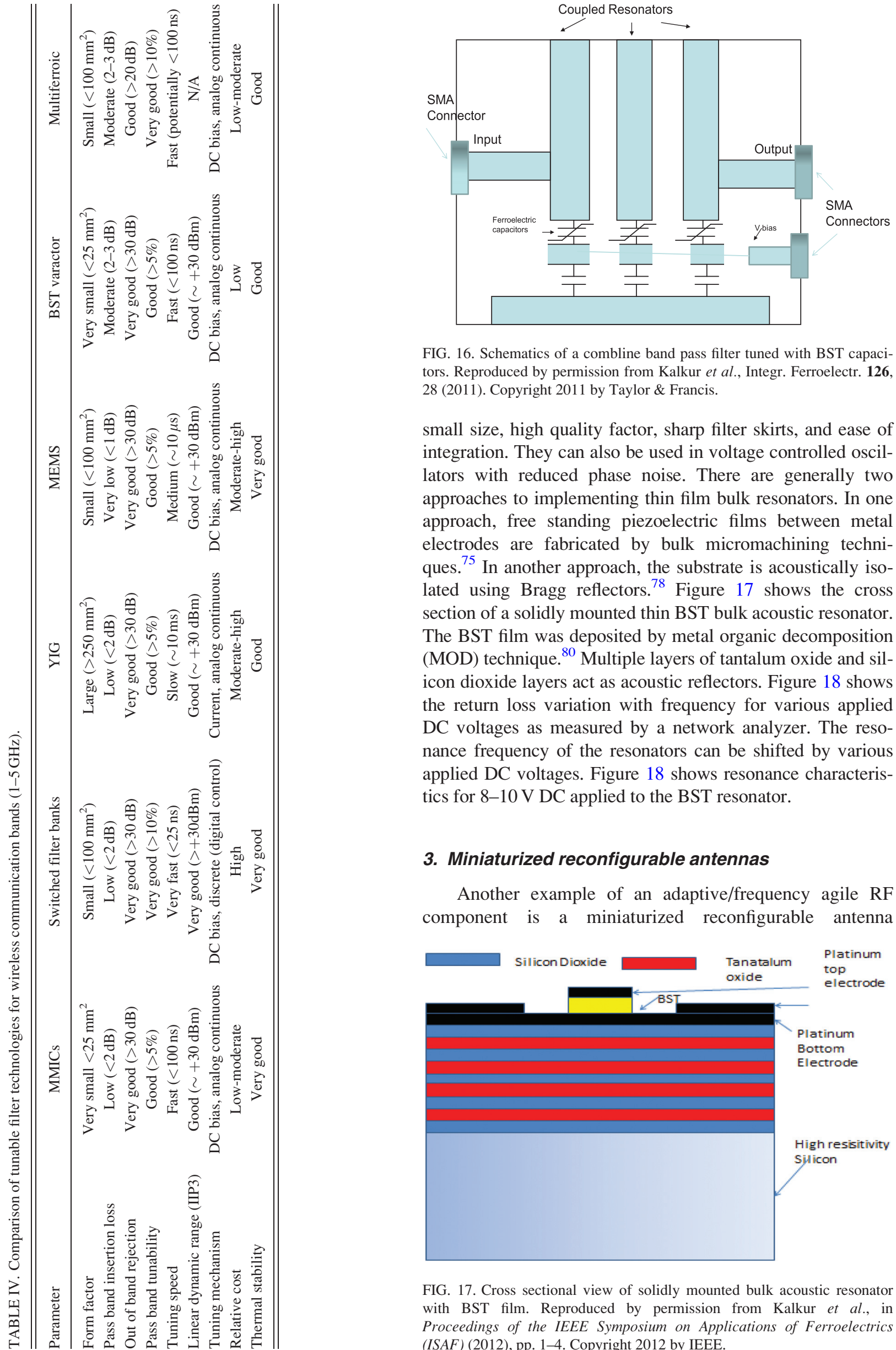

FIG. 16. Schematics of a combline band pass filter tuned with BST capacitors. Reproduced by permission from Kalkur et al., Integr. Ferroelectr. 126, 28 (2011). Copyright 2011 by Taylor \& Francis.

small size, high quality factor, sharp filter skirts, and ease of integration. They can also be used in voltage controlled oscillators with reduced phase noise. There are generally two approaches to implementing thin film bulk resonators. In one approach, free standing piezoelectric films between metal electrodes are fabricated by bulk micromachining techniques. $^{75}$ In another approach, the substrate is acoustically isolated using Bragg reflectors. ${ }^{78}$ Figure 17 shows the cross section of a solidly mounted thin BST bulk acoustic resonator. The BST film was deposited by metal organic decomposition (MOD) technique ${ }^{80}$ Multiple layers of tantalum oxide and silicon dioxide layers act as acoustic reflectors. Figure 18 shows the return loss variation with frequency for various applied DC voltages as measured by a network analyzer. The resonance frequency of the resonators can be shifted by various applied DC voltages. Figure 18 shows resonance characteristics for 8-10 V DC applied to the BST resonator.

\section{Miniaturized reconfigurable antennas}

Another example of an adaptive/frequency agile RF component is a miniaturized reconfigurable antenna

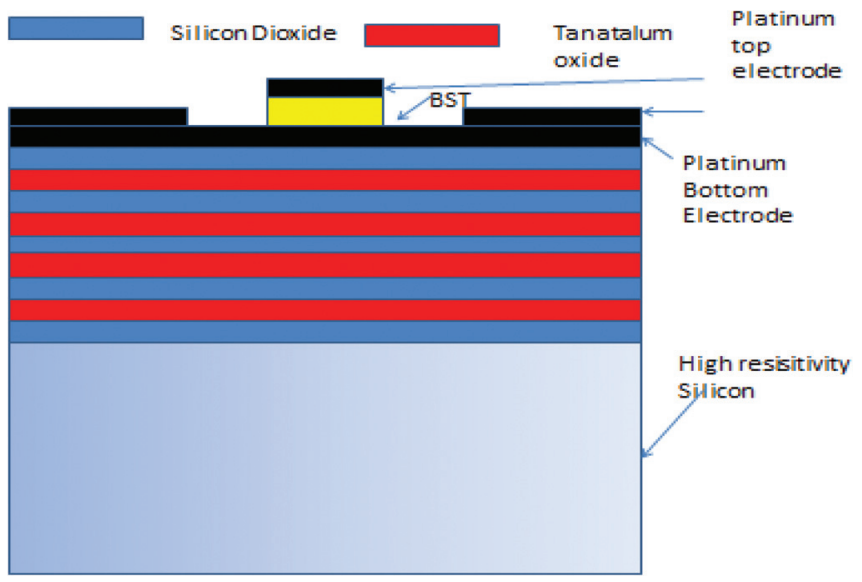

FIG. 17. Cross sectional view of solidly mounted bulk acoustic resonator with BST film. Reproduced by permission from Kalkur et al., in Proceedings of the IEEE Symposium on Applications of Ferroelectrics (ISAF) (2012), pp. 1-4. Copyright 2012 by IEEE. 


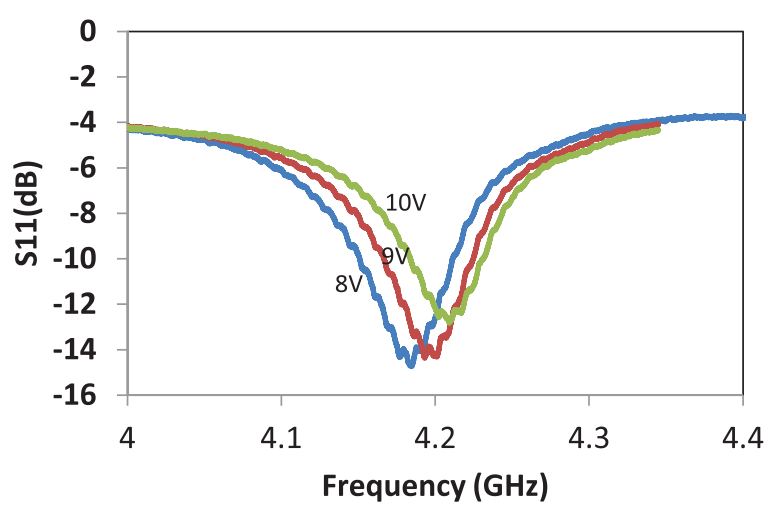

FIG. 18. Variation of return loss with frequency for solidly mounted thin film BST resonator for various DC applied voltages. The resonance frequency is tuned to higher frequency as one applies from $8 \mathrm{~V}$ to $10 \mathrm{~V}$. Reproduced by permission from Kalkur et al., in Proceedings of the IEEE Symposium on Applications of Ferroelectrics (ISAF) (2012), pp. 1-4. Copyright 2012 by IEEE.

demonstrated by Jiang et al. ${ }^{12}$ A patch antenna is loaded with BST varactors at the back end as shown in Figure 19. Using complex oxide BST thin film varactor technology, a miniaturized coplanar waveguide (CPW) square ring-slot antenna is successfully designed, fabricated and verified by experiments. The resonance frequency of the miniaturized antenna is also reconfigurable through applying DC voltages on the BST varactors. With loading of the BST varactors, no other higher resonances were observed for the antenna. Use of high dielectric constant BST thin film results in a reduced size antenna. Loading of the BST varactors allows for reconfigurability. The BST varactors are very compact in size and also require low DC voltage which saves power and reduces unwanted emitted radiation. This work demonstrates the complex oxide BST thin film varactor technology has great potential in antenna miniaturization and reconfiguration.

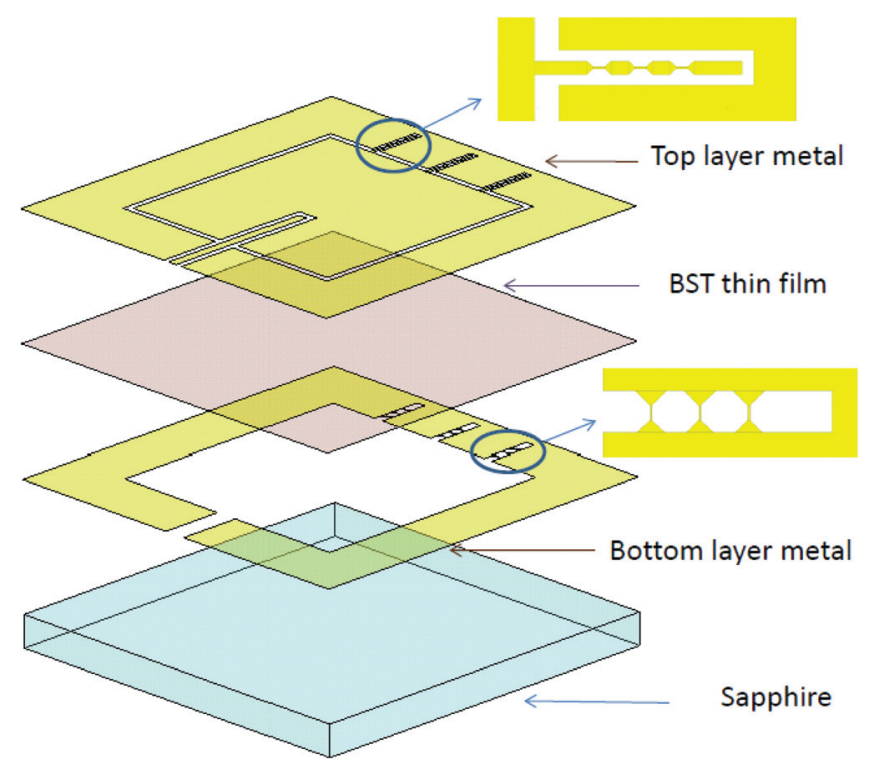

FIG. 19. Miniaturized and reconfigurable CPW square ring antenna with BST varactors loaded at the back. Reproduced by permission from Jiang et al., IEEE Trans. Antennas Propag. 60, 2111 (2012). Copyright 2012 by IEEE.

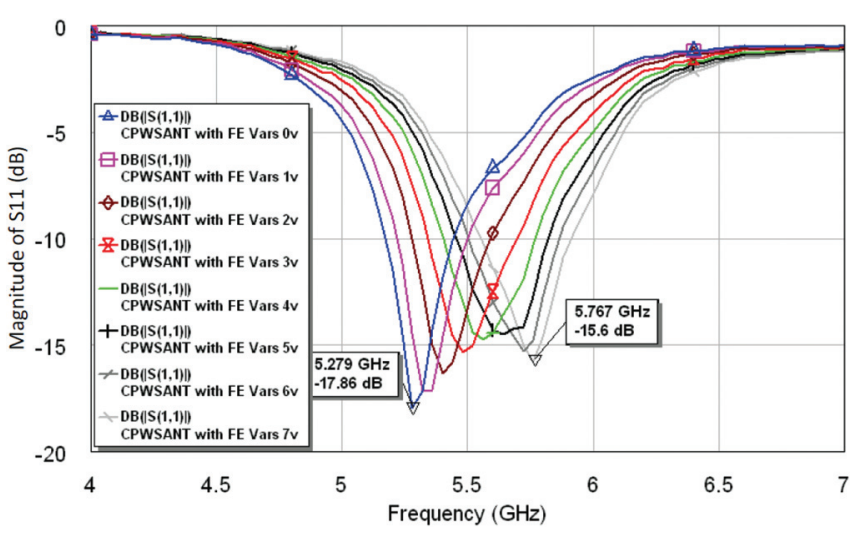

FIG. 20. Magnitude of the return loss for the antenna in Figure 10 for DC bias voltages 0 to $7 \mathrm{~V}$ showing the reconfigurability of the antenna with DC bias. Reproduced by permission from Jiang et al., IEEE Trans. Antennas Propag. 60, 2111 (2012). Copyright 2012 by IEEE.

The size of the miniaturized antenna is reduced to $0.067 \lambda_{0} \times 0.067 \lambda_{0}$ without ground, and $0.1 \lambda_{0} \times 0.1 \lambda_{0}$ with ground, where $\lambda_{0}$ is the free-space wavelength at the frequency of interest. The resonance frequency of the miniaturized antenna can be reconfigured from $5.3 \mathrm{GHz}$ to $5.8 \mathrm{GHz}$ by applying a DC voltage as shown in Figure 20. Note that the same patch radiates at $8 \mathrm{GHz}$ without the varactor loading. By combining the varactor, the antenna is forced to operate at lower frequencies, resulting in miniaturization. With the DC biasing of the varactors, the antenna exhibits reconfigurability.

\section{Adaptive electronics based on tunable BST capacitors}

\section{Impedance matching networks}

Adaptive RF/microwave systems are needed to make signal transmission and reception more immune to the environment. Matching networks or tuning networks (commonly referred as RF tuners) are used to optimize the performance of the amplifier by providing proper termination at the input and the output ports of the transistor. ${ }^{81}$ Typical impedance matching networks are designed for maximum power transfer between the source and the load in a communication system. Making these networks tunable/adaptive allows for higher efficiency of the system under all operating conditions. Simple matching networks are effectively low pass and high pass filters. A shunt capacitor or a series inductor acts as a simple low pass filter. A combination of these two elements creates a higher-order filter which has a sharper cutoff for the skirt of the filter. ${ }^{82}$ Figure 21 shows example topologies of the simple low pass filters used in matching networks.
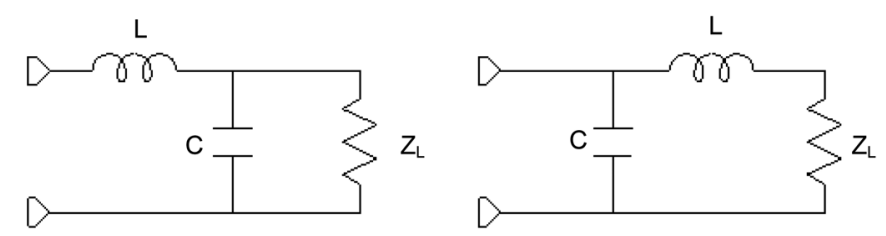

FIG. 21. Low pass filter topologies used in matching networks. 

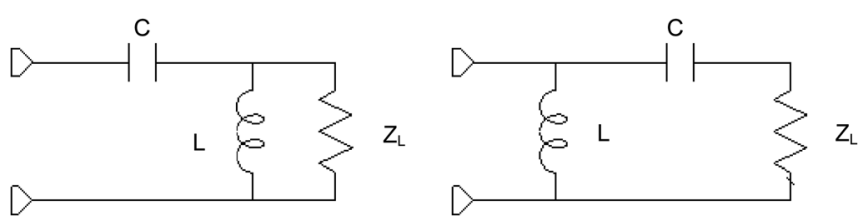

FIG. 22. High pass filter topologies used in matching networks.

A series capacitor or a shunt inductor acts as a simple high pass filter. Like the low pass filter, a combination of these elements will also create a higher-order filter which will have a sharper cutoff for the filter's skirt. Figure 22 shows example topologies of the high pass filters used in matching networks.

Band pass filters can also be used in matching networks. A band pass filter is a combination of a low pass and a high pass filter. This filter only allows a certain band of frequencies to pass and rejects all other frequencies. Figure 23 shows an example topology of the band pass filters used in matching networks.

When designing the matching networks, a Smith chart can be used to see the effects of the elements added to the matching networks. A shunt capacitor moves clockwise along a constant-conductance circle while a series capacitor moves counter-clockwise along a constant-resistance circle on the Smith chart. Similarly, a shunt inductor moves counter-clockwise along a constant-conductance circle while a series inductor moves clockwise along a constantresistance circle on the Smith chart. Figure 24 shows the effects of adding a series and a shunt element in matching networks on a Smith chart for a single frequency. If one uses a tunable inductor and a variable capacitor, one can cover a large portion of the Smith chart over a frequency range of interest.

The component values must be tunable over a wide range of values to cover variation of load impedance, the range of operation frequency, and harmonic rejection (filtering the higher order signals). For an amplifier design, it is important to maximize the gain produced by the amplifier to meet the gain requirements, by minimizing the impedance mismatches between the source and the amplifier and the amplifier and the load. The transducer gain, $G_{T}$, accounts for mismatch in the source and the load, and the associated reflection loss. It is easiest to define the separate effective gain factors for each of the three blocks of an amplifier shown in Figure 25 that affect the transducer gain.

Shunt capacitors can easily be implemented with BST varactor as shown in Figure 3. For circuits operating at

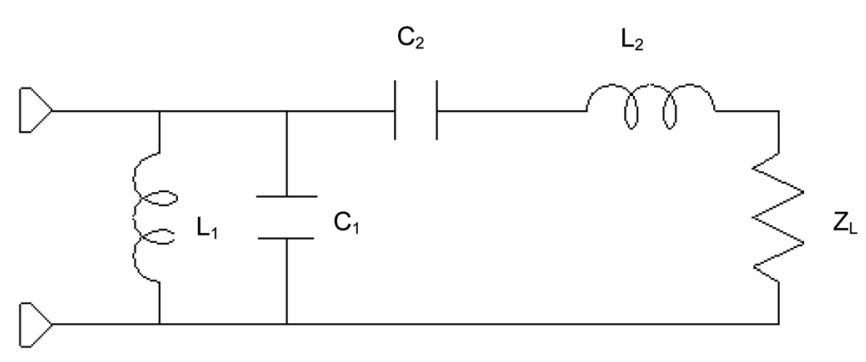

FIG. 23. Band pass filter topology used in matching networks.

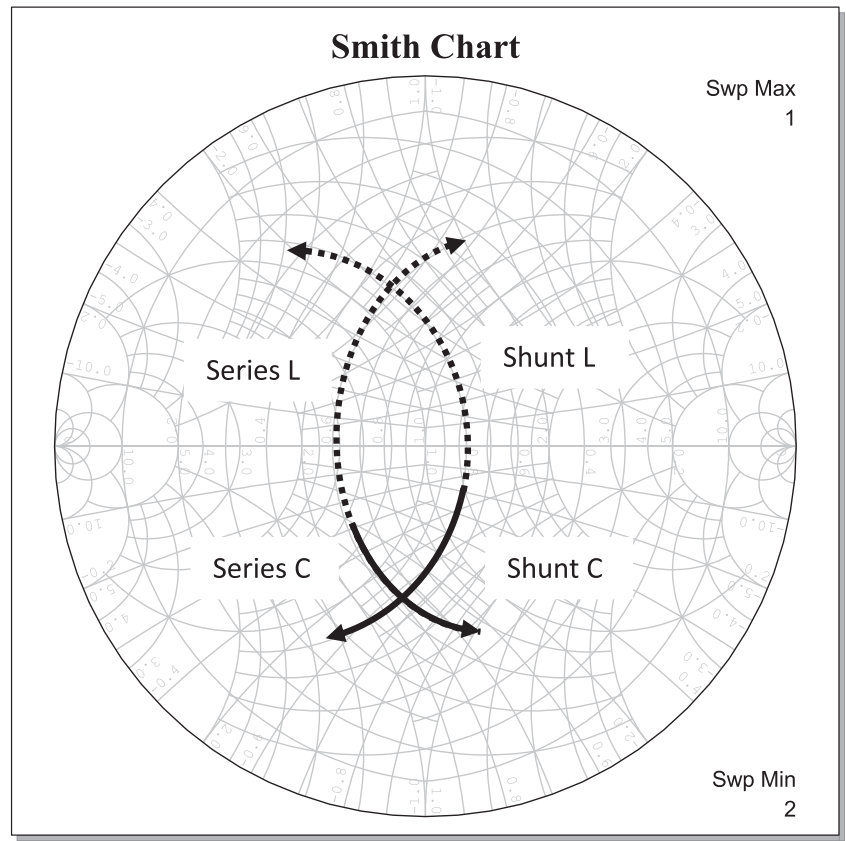

FIG. 24. Smith chart showing effects of adding series and shunt elements at one frequency.

frequencies above $10 \mathrm{GHz}$, series inductors can also be easily implemented using transmission lines. Figure 25 shows the schematics of a power amplifier with input and output matching networks with input and output coupling capacitors $\mathrm{Ci}$ and $\mathrm{Co}$ which are tunable BST capacitors. ${ }^{81} \mathrm{Ci}$ and $\mathrm{Co}$ can be tuned to match the source and load impedance, respectively.

Figure 26 shows the variation of gain of the power amplifier with the tunable bias voltage applied to $\mathrm{Ci}$. The unity gain frequency of the power amplifier can be tuned from $950 \mathrm{MHz}$ to $1.2 \mathrm{GHz}$ by applying a bias voltage of $4 \mathrm{~V}$ to the BST capacitor.

The amplifier gain and the bandwidth of the device can be altered using the varactor tuning as shown in Figure 26. Maintaining a desirable performance of an amplifier under any extreme condition will require tunable components like the varactors or variable inductors. Hence impedance matching is one of the key technology drivers for the oxide thin

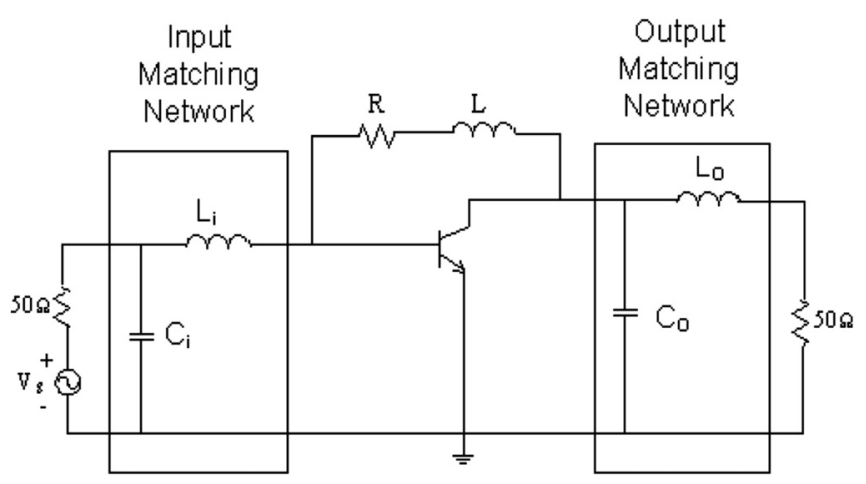

FIG. 25. Schematic of a power amplifier circuit with input and output matching networks. Reproduced by permission from Y. Zhang and T. S. Kalkur, in Proceedings of Progress in Electromagnetics Research Symposium (2011), p. 999. Copyright 2011 by The Electromagnetics Academy. 


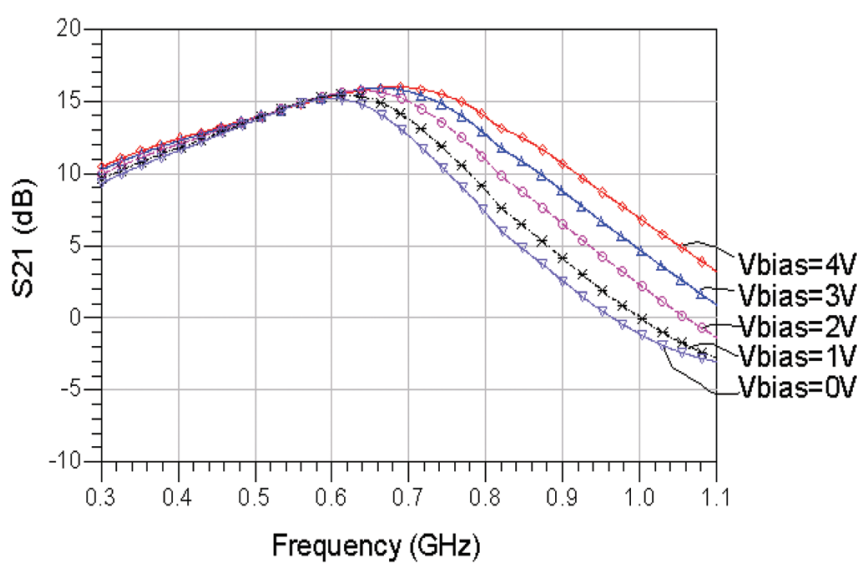

FIG. 26. Variation of amplifier gain with frequency for various bias voltages applied to BST capacitor. Y. Zhang and T. S. Kalkur, in Proceedings of Progress in Electromagnetics Research Symposium (2011), p. 999. Copyright 2011 by The Electromagnetics Academy.

film based varactors and multiferroic thin film based variable inductors.

\section{2. $\mathrm{VCOs}$}

VCOs are an integral part of communication systems. VCOs are widely used for applications in transmitters, receivers, synthesizers for avionics, wireless communication systems, satellites, instruments, and military electronics. In VCO implementations, high Q factor of ferroelectric varactor leads in turn to lower phase noise which is a critical factor for these circuits. ${ }^{80,81,83-87}$ Another requirement of $\mathrm{VCO}$ is the frequency tuning range and its linearity with respect to the control voltage. The VCO tuned with high dielectric constant ferroelectric capacitor based on Barium Calcium Zirconate Titanate (BCZT) was proposed and implemented by Kalkur et al. ${ }^{84}$ The frequency of oscillation for these differential VCOs are in the range of $2-5 \mathrm{MHz}$. Victor et al. used BST varactor to tune Colpitt VCO and the operation frequency of these VCOs are between $40-50 \mathrm{MHz}{ }^{85}$ Jamil et al. used BST varactors in differential VCOs and the configuration is shown in Figure 27. ${ }^{11}$

This VCO has a tuning range from $205 \mathrm{MHz}$ to 216.3 MHz with a power dissipation of $5.1 \mathrm{~mW}$. The measured phase noise is $-90 \mathrm{dBc} / \mathrm{MHz}$ at $100 \mathrm{kHz}$ and -140 $\mathrm{dBc} / \mathrm{Hz}$ at $1 \mathrm{MHz}$. Recently, a spread spectrum clock generator (SSCG) has been demonstrated with tunable BST capacitor based Colpitt VCO. ${ }^{86}$ Simple sinusoidal modulation resulted in a uniform spread in the energy with respect to central frequency of the oscillator as shown in Figure 28. SSCG based on $\mathrm{p}-\mathrm{n}$ junction varactor needs complicated modulating signals, compared to the BST varactors.

\section{Phase shifters for electronic steering of antenna arrays}

In general, present-day engineering technology investment for phased array antennas is focused on a mechanical phased-array antenna whereby "beam steering" is based on changes in mechanical motion to produce directional beam changes. Such mechanical systems are fairly simple to

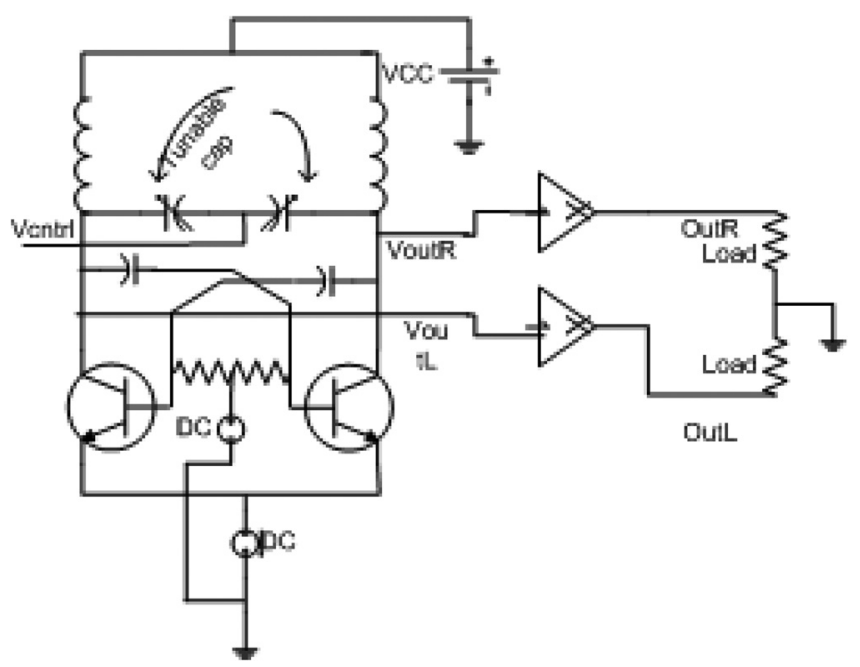

FIG. 27. Architecture of a differential VCO with BST capacitor as a varactor. Reprinted with permission from Jamil et al., IEEE Trans. Ultrason. Ferroelectr. Freq. Control 54, 222 (2007). Copyright 2007 IEEE.

implement and offer a low signal attenuation solution, however, such systems are large and bulky, slow response, not cost effective, and require high control powers. Systems engineers are aware of these technology drawbacks, hence the future desires for protected communication systems, $\mathrm{X}$ and $\mathrm{Ka}$ band SATCOM systems, is to replace such mechanical beam-steering architectures with Electronic Scanning Antenna (ESA) systems. As such, affordable, electronically scanned phased array antennas will serve to provide the means for achieving high data rate, beyond line of sight on-the-move (OTM) communications, essential for military battle-field operations. The phase shifter is a critical building block in the design of ESAs; it is the beam-steering component. Depending upon the application specifications of the OTM phased array systems, the number of phase shifters required for transmit and receive array to produce the

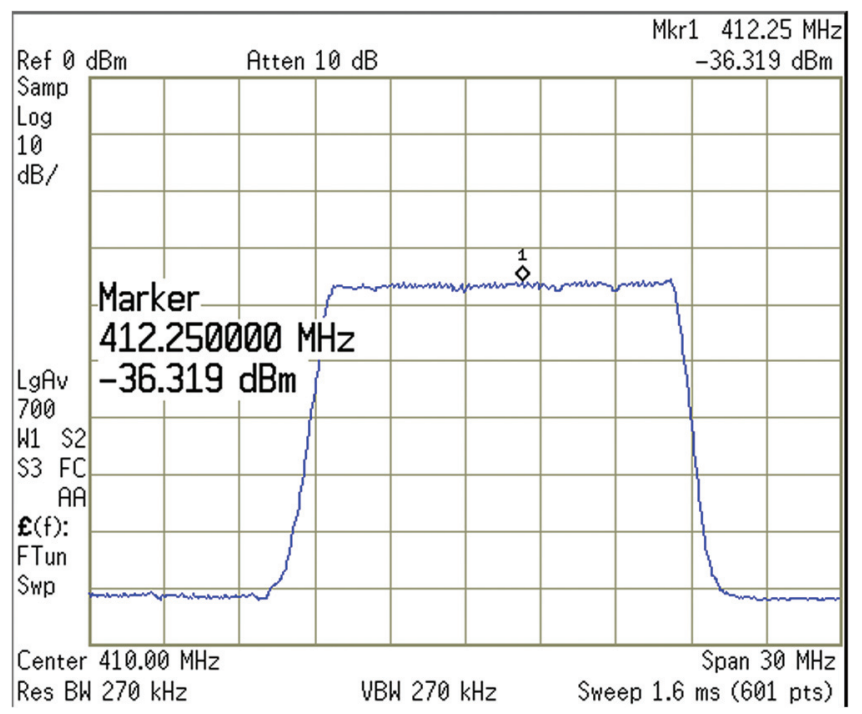

FIG. 28. Output power versus frequency for VCO tuned by BST showing significant reduction in peak power and the spreading of the signal power. Reprinted with permission from A. Kabir and T. S. Kalkur, IEEE Trans. Ultrason. Ferroelectr. Freq. Control 60, 1638 (2013). Copyright 2013 IEEE. 
electronic steering of the radiation pattern may range from several hundred to a thousand individual phase shifters. Unfortunately, the phase shifter is the most expensive element which composes these sub-arrays, hence the high cost of the phase shifter, combined with the large number of phase shifters for a given antenna system makes OTM arrays cost prohibitive. BST thin film varactor technology is a premier candidate for achieving affordable phase shifter elements.

The basic phase shifter application building blocks are the same as shown in Figure 1 and rely on the principle that the phase velocity of the wave propagating in the structures can be altered by changing the permittivity of the BST layer (via applied external bias), hence phase shifting is accomplished with respect to the zero-bias condition. ${ }^{9,87-92}$ The most popular implementation of phase shifters is using the distributed transmission line phase shifter (DTLPS), where the varactors are typically shunt loading the transmission lines periodically. ${ }^{9,89}$ By varying the capacitance, the phase velocity and hence the relative phase shift between the input and output can be accomplished. Typical phase shifters require both low insertion loss and high phase shift. Because of the periodic loading of varactors, the distributed phase shifter results in a pass-band and a stop-band. The frequency of interest needs to be in the pass band for low insertion loss. Another approach used for BST varactor based phase shifter is the coupled microstrip phase shifter (CMPS). ${ }^{90,91}$ CMPS consists of coupled microstrip lines with the coupling between the two lines defined by the separation of the two lines and the dielectric permittivity of the BST thin film. Compared to the parallel plate varactor approach, the interdigitated capacitor and the coupled microstrip line approaches need only a single layer of metal on top of the BST thin film. The electrode geometry is typically limited by the lithography to a few micron spacing, which results in high voltage application for the tuning of the CMPS and interdigitated capacitor phase shifters. The parallel plate varactors need less than $10 \mathrm{~V}$ typically for most applications. A figure of merit for phase shifters is the K-factor, the ratio of the phase shift obtained in degrees to the insertion loss of the device.

Table V compares the currently available phase shifter technologies. It is worth noting that the ferrite based phase shifters are obsolete and used sparingly for high power applications. Thin film BST phase shifters are attractive due to their low cost, and ease of processing compared to semiconductor monolithic microwave integrated circuit (MMIC) technology and the RF MEMS technology.

\section{E. Multiferroic devices for technology drivers}

Recent progress in multiferroic materials and devices include: (1) Signal processing applications for the frequency range of $1-77 \mathrm{GHz}$; (2) sensors at low frequencies of $10^{-3}-10^{3} \mathrm{~Hz}$; and (3) HF and UHF miniaturized antennas. Here, we summarize key materials and applications in these areas.

\section{Voltage tunable magneto-electric RF/microwave/millimeter wave devices}

There is a critical need for frequency tunable devices, such as band pass and bands top filters, resonators, phase shifters, and delay lines for next generation reconfigurable wireless communication systems and radars up to microwave and millimeter wave frequency regimes. These needs include conventional radar and signal processing devices as well as pulse based devices for digital radar and other systems applications. For secure systems, in particular, one must be able to switch rapidly between frequencies and to do so with a limited power budget. Traditional tuning methods with a magnetic field are too slow and power consumptive. Electric field or voltage tuning offers new possibilities to solve both problems.

Magnetic-piezoelectric heterostructures with strong converse ME coupling leads to effective voltage control of magnetism ${ }^{93-97}$ such as voltage tunable magnetic hysteresis loops, ${ }^{96}$ voltage tunable permeability, ${ }^{98}$ and voltage control of ferromagnetic resonance (FMR) frequency. ${ }^{97-108} \mathrm{~A}$ giant voltage tunable ferromagnetic resonance frequency from 1.7 to $7.6 \mathrm{GHz}$ observed in FeGaB/PZN-PT (lead zinc niobatelead titanate) is shown in Figure 29. ${ }^{97}$ This represents a promising new approach to build a new class of fast electric field tunable low power devices based on converse $\mathrm{ME}$ interactions. $^{24-26,97,98}$ An electric field $E$ applied to the piezoelectric transducer produces a mechanical deformation that couples to the ferrite and produces a change in the ferromagnetic resonance field, ferromagnetic resonance frequency, or permeability. Unlike the situation when magnetic fields are used for such tuning, the voltage tuning process is fast, and power efficient as the biasing voltages involve minimal currents and are nearly passive. This process has been demonstrated in voltage tunable bandpass filters, ${ }^{27}$ voltage tunable bandstop filters, ${ }^{26}$ voltage tunable delay lines, ${ }^{93}$ and voltage tunable inductors. ${ }^{23}$ Figure 30 shows the schematic of such a voltage tunable ferromagnetic resonance based tunable bandstop filter. ${ }^{26}$ The critical goal for the future is in the

TABLE V. Comparison of phase-shifter technologies for microwave applications in the 1-20 GHz range.

\begin{tabular}{|c|c|c|c|c|}
\hline Parameter & MMICs technology & Ferrite technology & MEMS & BST varactor \\
\hline Form factor & Very small $<25 \mathrm{~mm}^{2}$ & Large $\left(>250 \mathrm{~mm}^{2}\right)$ & Small $\left(<100 \mathrm{~mm}^{2}\right)$ & Very small $\left(<25 \mathrm{~mm}^{2}\right)$ \\
\hline Maximum insertion loss & Low $(<3 \mathrm{~dB})$ & Low $(<2 \mathrm{~dB})$ & Very low $(<1 \mathrm{~dB})$ & Moderate (4-6 dB) \\
\hline Phase-shift (range) & $0^{\circ}-360^{\circ}$ & $0^{\circ}-360^{\circ}$ & $0^{\circ}-360^{\circ}$ & $0^{\circ}-360^{\circ}$ \\
\hline Tuning speed & Fast $(<100 \mathrm{~ns})$ & Slow $(\sim 10 \mathrm{~ms})$ & Medium $(\sim 10 \mu \mathrm{s})$ & Fast $(<100 \mathrm{~ns})$ \\
\hline Tuning mechanism & DC bias, analog continuous & Current, analog continuous & DC bias, analog continuous & DC bias, analog continuous \\
\hline Relative cost & Low-moderate & Moderate-high & Moderate-high & Low \\
\hline Thermal stability & Very good & Good & Very good & Good \\
\hline Figure of merit (K-factor) & $>120^{\circ} / \mathrm{dB}$ & $>120^{\circ} / \mathrm{dB}$ & $>120^{\circ} / \mathrm{dB}$ & $70^{\circ} / \mathrm{dB}$ (typical) \\
\hline
\end{tabular}




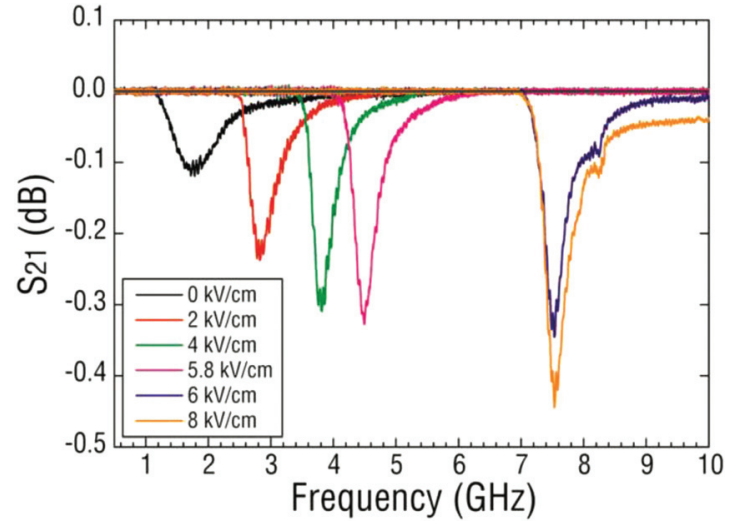

FIG. 29. Transmission coefficient $\left(\mathrm{S}_{21}\right)$ spectra for a FeGaB/PZNPTmultiferroic heterostructure measured by a network analyzer. Reproduced by permission from Lou et al., Adv. Mater. 21, 4711 (2009). Copyright 2009 by John Wiley \& Sons.

development of a new class of compact, power efficient, wide tunable range, and low loss electrically tunable magnetic film devices for radar and wireless communication devices. The anticipated advantages of ME devices are yet to be exploited.

The focus need to be on three classes of multiferroic $\mathrm{RF} /$ microwave/millimeter devices based on converse $\mathrm{ME}$ coupling, (1) those based on the voltage tuning of the FMR response, (2) those based on hybrid spin-wave electromagnetic wave tuning, and (3) those based on voltage tunable permeability. Class 1 devices could include tunable band pass and bandstop filters, ${ }^{26,27}$ for example. Class 2 devices could include spin-wave delay lines, spin-wave soliton pulse cloning devices, ${ }^{22}$ and broadband chaos microwave signal generators ${ }^{29}$ among others. Class 3 devices include voltage tunable multiferroic inductors, ${ }^{23}$ and voltage tunable bandpass filters and phase shifters based on partially magnetized ferrites. ${ }^{101-108}$ For this device development, a four track approach should be considered, based on (a) materials selection, structure development and fabrication, (b) characterization, (c) device design and simulation, optimization of the ME tuning, and (d) device physics and engineering.

\section{Low frequency sensors and sensor networks $\left(10^{-3}-10^{3} \mathrm{~Hz}\right)$}

New generations of magnetic field sensors are required for the national defense. Specific examples include (but not
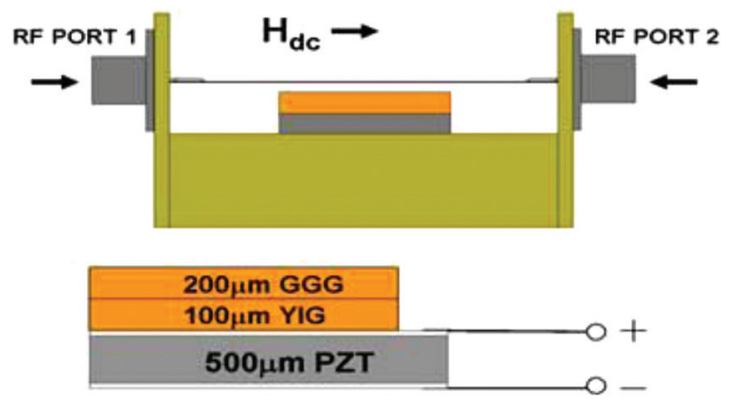

FIG. 30. Example of a magnetic film (YIG) PZT electric field tunable microwave magnetic strip line device. Reproduced by permission from Pettiford et al., IEEE Trans. Magn. 43, 3343 (2007). Copyright 2007 by IEEE. limited to) anti-submarine warfare (ASW) surveillance systems that are capable of monitoring all littoral water activity. Sensor networks distributed in key areas of the ocean could play an important role in the collection of data to track merchant vessels and possible maritime threats, detection of mines and explosive devices carried by humans/animals/ vehicles, counter-Improvised Explosive Devices (IED), and examination of shipping containers and trucks. The magnetic sensors in most common use today are variable reluctance coils and Hall-effect devices. The drawback of variable reluctance sensors is that they generate a signal proportional to the magnetic field's rate of change. Signal strength therefore decreases with decreasing speed, and below a certain flux change rate, the signal disappears into the noise. The excess output voltage of the coil at high-frequency magnetic fields also causes problems for circuit designers. Hall effect devices generate a very small raw signal because of low field sensitivities $(0.5-5.0 \mathrm{mV} / 100 \mathrm{Oe}$ applied field), and the device performance is strongly temperature dependent. These features mandate signal conditioning, and require that a certain minimum field be available for device operation.

The development of new sensors and their networks is a critically enabling technology. The key challenges or requirements of magnetic field-sensors are (i) extreme sensitivity $\left(\sim \mathrm{pT} / \mathrm{Hz}^{1 / 2}\right)$, allowing for better anomaly detection capabilities and also a wider sensor spacing; (ii) zero power consumption-as passive as possible-in order to foster longevity of deployment; (iii) operation at low frequencies, in order to detect the motion of potential threats; (iv) miniature size, enabling the deployment of literally thousands of such sensors over a strategic information space; and (v) low cost of manufacturing, in order to make the technology realizable for operational command. The proposed ME sensors are based on magneto-electric effect in ferromagnetic/piezoelectric composites. Such sensors are truly passive, a sensitivity of $10 \mathrm{mV} / \mathrm{Oe}$, and would require minimal signal conditioning.

Recently, a new generation of single element ME laminate composite magnetic field sensors has been developed. ${ }^{109,117,118}$ The ME effect offers an alternative passive approach to magnetic field sensing and the effect is largest for composites consisting of piezoelectric and magneto-strictive layers laminated together, in either longitudinal-transverse (L-T) or longitudinallongitudinal (L-L) vibration modes. At $1 \mathrm{~Hz}$, the sensitivity limit to minute magnetic field variations was about $5 \mathrm{pT} / \mathrm{Hz}^{1 / 2}$. It is possible to (i) develop small ME sensors that have pico-Tesla resolution over the bandwidth of $10^{-3}<\mathrm{f}<10^{4} \mathrm{~Hz}$, that are integrated with detection circuitry enabling autonomy, that are appropriate encapsulated, and that have optimized signal-noise $(\mathrm{S} / \mathrm{N})$ ratios; (ii) develop arrays of miniaturized ME sensors and gradiometers; and (iii) demonstrate operational applicability for Department of Defense needs.

Several research teams have recently developed a new generation of multiferroic magnetic sensors. ${ }^{109,117,118} \mathrm{~A}$ majority of these are made of ferromagnetic and ferroelectric layers. The coupling between the two subsystems, termed ME interaction, is mediated by mechanical strain. Magnetic sensors with Metglas or 3d-transition metals/alloys for the magnetic phase and PZT or PMN-PT for the ferroelectric phase are reported to show a noise floor on the order of 5-10 
$\mathrm{pT} /{ }_{\sqrt{ } \mathrm{Hz}}$ at $1 \mathrm{~Hz} \cdot{ }^{117}$ But such sensitivities are achieved only when a DC bias $H$ is present. Thus, current status of ME sensors have several drawbacks including (i) large size $(5-8 \mathrm{~cm}$ long laminates) ${ }^{117}$ (ii) need for dc bias, (iii) potential nonlinear response due to ferroelectric hysteresis, and (iv) noise limitations due to ferroelectric losses and pyroelectric effects. Some recent studies show comparable sensitivities in miniature sensors of piezoelectric AlN-ferromagnetic alloys. ${ }^{109,118}$

\section{Broadband miniaturized antenna structures utilizing magneto-electric materials}

Antenna miniaturization has been the subject of interest for more than half a century, but in recent years, it has received significant attention because of additional demand for numerous mobile communication systems on a single platform. The need for miniaturization is even more obvious for the lower frequency bands where the wavelength is larger. At low frequencies, as with the HF and UHF bands, a standard half-wavelength dipole is in the range of a few meters in length. This is prohibitively large. Low frequency carriers, however, are suitable choices for scenarios where line of sight communication is unlikely, including urban environments, vegetation canopies or irregular terrains. The larger wavelength at lower frequencies makes it possible for electromagnetic waves to propagate through canopies and around bends and curvatures via edge diffraction. A miniaturization solution for antennas is inevitable. The goal is to obtain a compact topology that resonates at the same resonant frequency as that of the standard resonant dipole with the same partial standing wave pattern.

We describe an approach to drastically reduce the size of antenna elements using magneto-dielectric materials and slow-wave topologies. ${ }^{110-116}$ The special case of a magnetodielectric material, when the permittivity $\varepsilon$ and permeability $\mu$ are equal, is sought. In this case, the characteristic impedance of the medium is equal to that of the free-space. As a result, no reflections take place between the finite substrate of the antenna and the surrounding free space, thereby reducing the energy trapped in the substrate. The above statement is theoretically valid only for the normal incidence but for most practical purposes may hold for angles far from grazing incidence. Given that the antenna is properly designed, such that the radiating fields do not impinge on the substrate/air interface at grazing incidence, one can expect that the stored energy in the near-field of the antenna will be drastically reduced. A lower stored energy in the near-field of the antenna is equivalent to a lower $Q$ and a wider bandwidth. A substrate with $\mu=\varepsilon$ is also the perfect case for a reduction in the surface wave excitation in antenna, again due to the impedance match between the meta-substrate and the surrounding air.

\section{CHALLENGES FOR MULTIFUNCTIONAL OXIDE AND MULTIFERROIC THIN FILM BASED TECHNOLOGIES}

\section{A. Material requirements for RF/microwave applications}

Although BST-based frequency agile circuits, such as tunable filters, and phase shifters offer good performance attributes, there are still some major caveats that are impeding their progress as a technology solution for many applications. Critical materials requirements for BST thin films utilized in microwave applications are shown in Table VI.

To date, researchers have fabricated BST-based thin films that satisfy many of the dielectric and insulating performance criteria, listed in Table VI. It is well known that the dielectric and insulation properties of BST films are strongly influenced by the growth technique employed. Hence, a fabrication process that can reproducibly grow high quality BST thin films (smooth and dense with minimal defects) with good control of material properties over large area substrates is needed to obtain enhanced dielectric/electrical properties that are consistent across a single wafer and between wafer lots. A viable material growth/deposition process must have a low deposition temperature to be compatible semiconductor process protocols and to reduce interaction between film and substrate/bottom electrode; produce

TABLE VI. Critical materials requirements for BST thin films utilized in varactors and other microwave applications.

\begin{tabular}{|c|c|c|}
\hline Performance limiting property & Materials property required & Device property affected \\
\hline Dielectric tunability & $\begin{array}{l}\text { High tunability } \Delta \mathrm{C} / \mathrm{C}_{\mathrm{o}}>50 \% \text { or }>2: 1 \text { ( } \Delta \mathrm{C} \text { is the change in } \\
\text { capacitance relative to zero-bias capacitance } \mathrm{Co} \text { ) }\end{array}$ & Tuning bandwidth, device phase shift \\
\hline Leakage current & Low leakage characteristics $\mathrm{I}_{\mathrm{L}} \sim 10^{-7} \mathrm{~A} / \mathrm{cm}^{2}$ & Reliability, battery draw, power consumption \\
\hline Dielectric permittivity & Moderate, $\varepsilon_{\mathrm{r}}<500$ & Impedance matching, (efficient power transfer in the device) \\
\hline Dielectric loss-tangent & Low Loss: $\tan \delta<2 \%$ over operating dc bias voltage range & Device insertion loss, dielectric quality factor \\
\hline Conductor loss & Low surface resistance at frequency of interest & Device insertion loss, conductor quality factor \\
\hline Dielectric temperature stability & $\begin{array}{l}\text { Low TCC: } \mathrm{TCC}<200 \mathrm{ppm} /{ }^{\circ} \mathrm{C} \mathrm{TCC}=\Delta \mathrm{C} /\left(\mathrm{C}_{\mathrm{o}} \Delta \mathrm{T}\right)(\text { broad } \\
\text { flat dielectric response over required operating temperature })\end{array}$ & $\begin{array}{l}\text { Reliability of frequency agile circuits, impedance matching } \\
\text { networks, and phase shifters }\end{array}$ \\
\hline Dielectric bias reproducibility & $\begin{array}{l}\text { Maximum reproducibility of dielectric response with respect } \\
\text { to applied dc bias }\end{array}$ & $\begin{array}{l}\text { Reliability of frequency agile circuits, impedance matching } \\
\text { networks, and phase shifters }\end{array}$ \\
\hline $\begin{array}{l}\text { Structure, surface, and } \\
\text { microstructural properties }\end{array}$ & $\begin{array}{l}\text { - Structurally, single phase film } \\
\text { - Uniform composition, thickness, and Microstructure } \\
\text { with minimum defects across the wafer }\end{array}$ & $\begin{array}{l}\text { Reliability } \\
\text { Reliability insertion loss }\end{array}$ \\
\hline & - Smooth surface morphology $\left(\mathrm{R}_{\mathrm{a}}=<2 \mathrm{~nm}\right)$ & Insertion loss \\
\hline
\end{tabular}




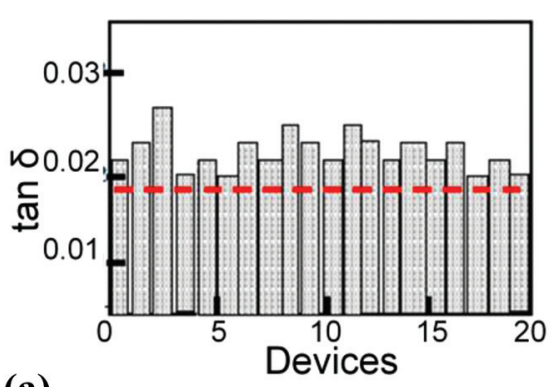

(a)

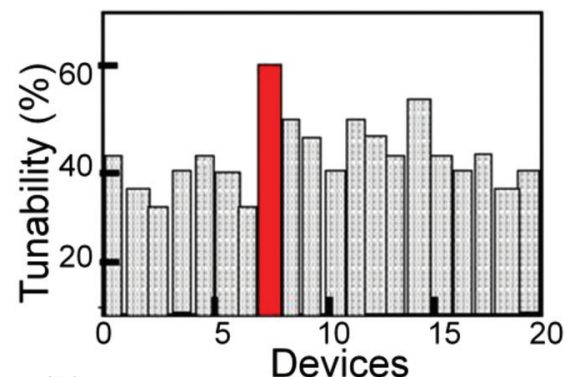

(b)
FIG. 31. An example of tunable capacitor performance inconsistency with respect to (a) loss and (b) capacitor tunability. The dashed line in (a) represents uniform loss and the solid bar in (b) represents the optimum device tunability (Courtesy of M. W. Cole research group, Army Research Laboratory 2012). excellent film crystallinity to improve device performance; possess high growth rate, and be capable of large area uniform deposition in order to promote scale up and low cost manufacturing.

\section{Effect of thickness uniformity of oxide thin films}

The ability to fabricate BST-based thin films with uniform material properties is critical for insertion into various microwave applications. In particular, the uniformity of the dielectric and insulation properties (at microwave frequencies), such as capacitance density, which is correlated to capacitor tunability and the device Q across the wafer. Figure 31 shows the device loss and tunability for a typical set of varactor test structures fabricated on a single wafer. The results shown in Figure 31 clearly demonstrate the extreme non-uniformity in both loss and tunability of typical BST based varactors. Ideally, a low and uniform loss (dashed line in Figure 31(a)) and a high degree of capacitor tunability (solid red bar in Figure 31(b)) for all devices fabricated on a single wafer and between wafer lots is preferred. The effect of materials property uniformity across the wafer is further illustrated in Figure 32 by comparing the phase shift and loss from devices fabricated at the center and edges of a typical large area wafer. In this example, the BST film is thicker in the center than near the edge of the wafer. This has a profound effect on the device properties such that the devices fabricated near the center of the wafer would possess a different capacitance density, hence different degrees of phase shift than those devices located near the edge of the wafer. In other words, the BST film thickness variations would manifest into phase shifter device non-uniformity such that the devices fabricated from this wafer would possess a variety of different degrees of phase shift, hence the devices would not perform equally. Similarly, non-uniformity in other materials properties, such as composition ( $\mathrm{Ba} / \mathrm{Sr}$ ratio), microstructure, stress, defects, and surface roughness, also compromise the phase shifter device property uniformity (Figure 33).

Higher $\mathrm{Ba} / \mathrm{Sr}$ ratios yield higher tunability/phase shift and higher loss. Microstructural variations in grain size strongly affect the permittivity/impedance matching and tunability/phase shift. Large grains yield higher permittivity and tunability. Rough surfaces augment the conductor loss. The concentration of defects, such as oxygen vacancies, increases the dielectric loss. The film stress also strongly affects the BST materials properties (tunability, permittivity, and loss), hence affects the tunable device properties. Thus, development of consistent and predictable tunable device performance requires the development of "wafer scale" BST film property control and uniformity. ${ }^{118-121}$

To date, the best capacitance tuning ratio, highest $Q$ factor and the K-factor have been obtained on sapphire substrates, with the thickness of BST layer $>0.5 \mu \mathrm{m}$, and bottom metal layer stack of Ti/Pt/Au/Pt. ${ }^{123}$ Subramanyam et al. studied the effect of BST layer thickness on the varactor devices with the metal layers kept the same, and the geometry of the parallel plate varactor devices kept the same. ${ }^{123}$ The top metal layer stack is typically Ti/Pt/Au. Bottom and top metal layer stack are typically $1 \mu \mathrm{m}$ in thickness. Figure 34 shows the capacitance vs. electric field for the three different BST film thicknesses studied. The thin film properties depend highly on the thickness of the BST layer.

Figure 34 shows that the thicker BST thin film layer results in higher capacitance tunability at a fixed electric field. Based on the capacitance at zero-bias, the $0.6 \mu \mathrm{m}$ and $1 \mu \mathrm{m}$ BST varactors had a zero-bias relative dielectric constant $\sim 1280$ and tuned more than 5:1 with dc bias, with the lowest value $\sim 220$ at $20 \mathrm{~V}$. Also, the $\mathrm{Q}$ of the varactors have thickness dependence as well, as thicker BST layers have yielded higher Q devices, as shown in Figure 35. The thicker

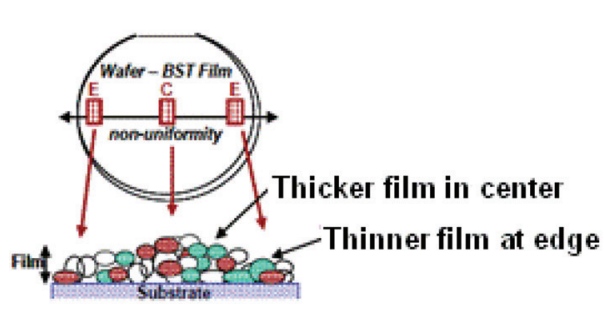

(a)

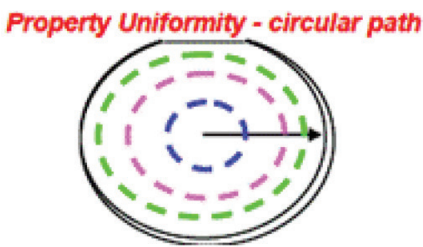

Tight fit of device properties along
concentric circular paths

Selectivity in properties increases cost
FIG. 32. Wafer level device non-uniformity: (a) film thickness variation across the wafer which results in low device yield whereby the devices with equivalent film thickness/capacitance density (phase shift) form radial paths as shown in (b) (Courtesy of M. W. Cole research group, Army Research Laboratory 2012). 


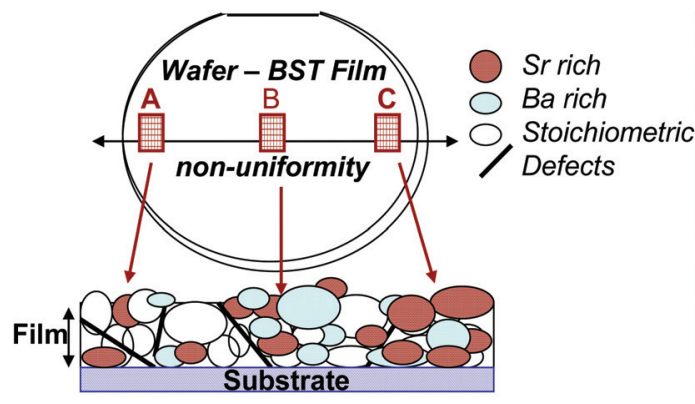

Loss varies as a function of device position on wafer

$>$ Composition non-uniformity [high $\mathrm{Ba} / \mathrm{Sr}$ inc. loss]

$>$ Surface roughness [rough surfaces inc. loss]

$>$ Thickness non-uniformity [very thin films inc. loss]

$>$ Stress non-uniformity [large stress inc. loss]

$>$ Defect concentration $\left[\mathrm{O}_{2}\right.$ vacancy inc. loss]

Phase Shift as a function of device position on wafer

$>$ Grain size non-uniformity [small grains low $\varepsilon_{r}$ low tunability]

$>$ Stress non-uniformity [high stress dec. tunability]

$>$ Composition non-uniformity [high $\mathrm{Ba} / \mathrm{Sr}$ inc. tunability

$>$ Thickness non-uniformity [thin film high $\mathrm{C}_{\mathrm{p}}$ /high tunability

FIG. 33. Schematic diagram showing (a) the film compositional and microstructural non-uniformities and (b) their effects on the phase shifter device properties (Courtesy of M. W. Cole research group, Army Research Laboratory 2012).

BST layer results in a higher leakage current at a fixed electric field $(200 \mathrm{kV} / \mathrm{cm})$ as shown in Figure 36. It is clear that the dielectric properties, the dielectric tunability, and Q are enhanced by the growth of thicker BST layers. The Q for the thicker BST layer varactors exceed commercially available GaAs varactors. Considering the improvement in dielectric tunability and the Q factor, $0.5-0.6 \mu \mathrm{m}$ BST varactor could be attractive for its practicality, as $1 \mu \mathrm{m}$ thick BST layer requires close to 80000 laser pulses, and longer time to grow utilizing the PLD system, which may be impractical.

\section{Reducing dielectric loss-tangent in oxide thin films}

The loss-tangent needs to be below 0.02 at $\mathrm{GHz}$ frequencies for all dc voltage bias for useful microwave applications of these devices. Reducing the loss-tangent will improve the dielectric quality factor. Attempts to reduce the dielectric loss in BST films include, acceptor doping (e.g., $\mathrm{MgO}, \mathrm{Al}_{2} \mathrm{O}_{3}, \mathrm{ZrO}_{2}$, etc.), ${ }^{124,125}$ introduction of buffer layers to reduce mismatch strain due to lattice and/or coefficient of thermal expansion (CTE) mismatch ${ }^{126,127}$ utilization of conducting oxide electrodes $\left(\mathrm{LSCO}, \mathrm{SrRuO}_{3}, \mathrm{RuO}_{2}\right)^{128}$ or thick Au bottom electrodes, ${ }^{122,129,130}$ inter-layering of the BST with a low loss (low microwave dielectric loss tangents)

\section{Tunability of BST Varactors}

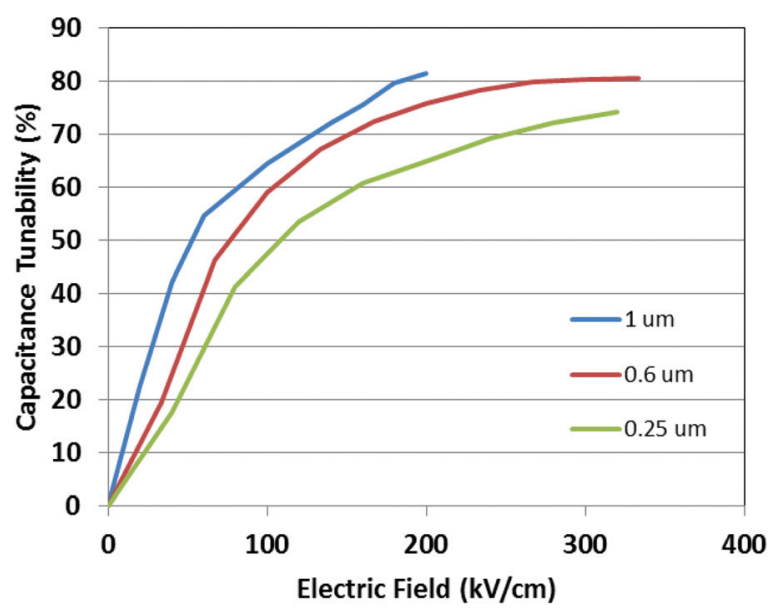

FIG. 34. Thickness dependence of capacitance tunability vs. electric-field for $5 \mu \mathrm{m} \times 5 \mu \mathrm{m}$ size varactor. Reproduced by permission from Subramanyam et al., Integr. Ferroelectr. 112, 53 (2009). Copyright 2009 by Taylor \& Francis. linear dielectric $\left(\mathrm{MgO}, \mathrm{Ta}_{2} \mathrm{O}_{5}, \mathrm{Al}_{2} \mathrm{O}_{3}\right.$, etc.) and/or low loss pyrochlore $\left(\mathrm{Bi}_{1.5} \mathrm{ZnNb}_{1.5} \mathrm{O}_{7} / \mathrm{BZN}\right)$ films, ${ }^{129-132}$ and tuning of growth and/or post deposition crystallization ambience/temperatures. ${ }^{122,126-132}$ Although these methods have been fairly successful at reducing dielectric loss, this property enhancement has often been accomplished at the expense of degrading other required properties (tunability, leakage current requirements, etc.), added complexity and/or film processing steps which are often not complementary metal oxide semiconductor (CMOS) compatible, hence limit their potential. In addition to the independent optimization of the dielectric loss and tunability it is necessary to balance these two properties, i.e., to attain simultaneous minimization of the films dielectric loss (minimization of signal attenuation) in conjunction with maximization of films dielectric tunability. This is a difficult challenge as two properties are tightly coupled and negatively opposed to one another.

Recently, success has been achieved in balancing these two material properties by developing and optimizing an isothermal ultraviolet (UV) photon irradiation process science protocol which served to mitigate the oxygen vacancies (responsible for the high losses in the BST films), and reduced the dielectric loss without degrading the dielectric tunability. ${ }^{133}$ This work demonstrated a non-complex method to decouple the inverse relationship between dielectric tuning and dielectric $Q(1 / \tan \delta$ ) (property interdependence) and

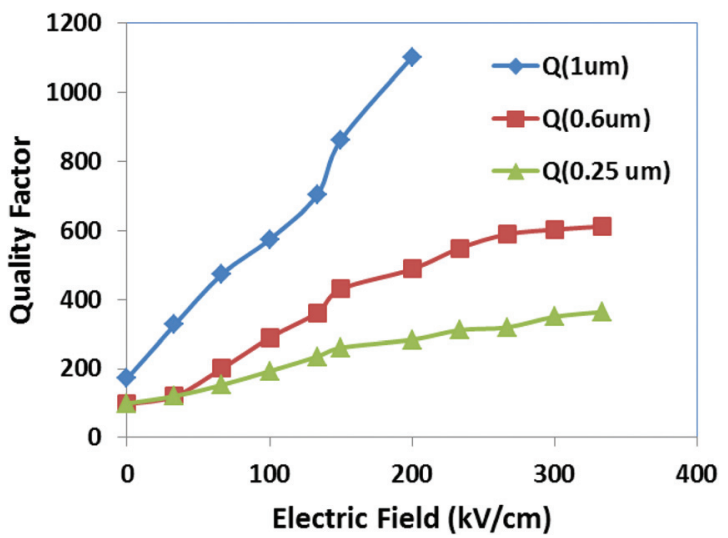

FIG. 35. Quality factor vs. electric field for $5 \times 5 \mu \mathrm{m}^{2}$ varactors fabricated using 3 different thicknesses of BST layers. The Q was computed at $1 \mathrm{GHz}$. Reproduced by permission from Subramanyam et al., Integr. Ferroelectr. 112, 53 (2009). Copyright 2009 by Taylor \& Francis. 


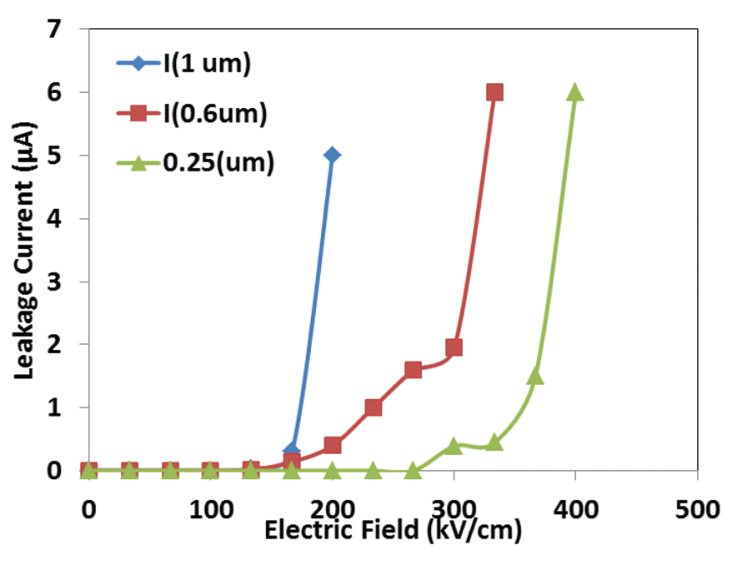

FIG. 36. Leakage current vs. electric field for the same $5 \mu \mathrm{m} \times 5 \mu \mathrm{m}$ varactors as a function of BST layer thickness. Note that the leakage current starts increasing at a lower dc electric field for the thicker layers. Reproduced by permission from Subramanyam et al., Integr. Ferroelectr. 112, 53 (2009). Copyright 2009 by Taylor \& Francis.

achieve enhanced dielectric response. The experimental results demonstrated significantly improved material properties for the UV-photon irradiation treated BST films. Specifically, $20 \%$ reduction in loss was observed for the UV-photon irradiation treated films with respect to a nontreated/control film (Figure 37). The UV-treated films also exhibited a three-order of magnitude reduction in leakage current density as compared to the non-UV-treated control film (at $\mathrm{E}=500 \mathrm{kV} / \mathrm{cm}$ the leakage current density of the non-UV treated BST film was $1.510 \times 10^{-3}$ vs. 4.609 $\times 10^{-6} \mathrm{~A} / \mathrm{cm}^{2}$ for the $225 \mathrm{~min} \mathrm{UV}$ treated film) without degrading dielectric tunability. ${ }^{133}$ What is most impressive about this post-growth UV-processing method is not only did it significantly improve the dielectric loss and leakage current characteristics; it also served to elevate the useable tunability by a factor of two with respect to traditionally fabricated BST films, i.e., $>50 \%$ (Figure 38). A mechanistic model suggested that the formation of reactive oxygen species generated by the UV-irradiation source, are absorbed on the film surface and diffuse into the film, accept electrons and occupy the

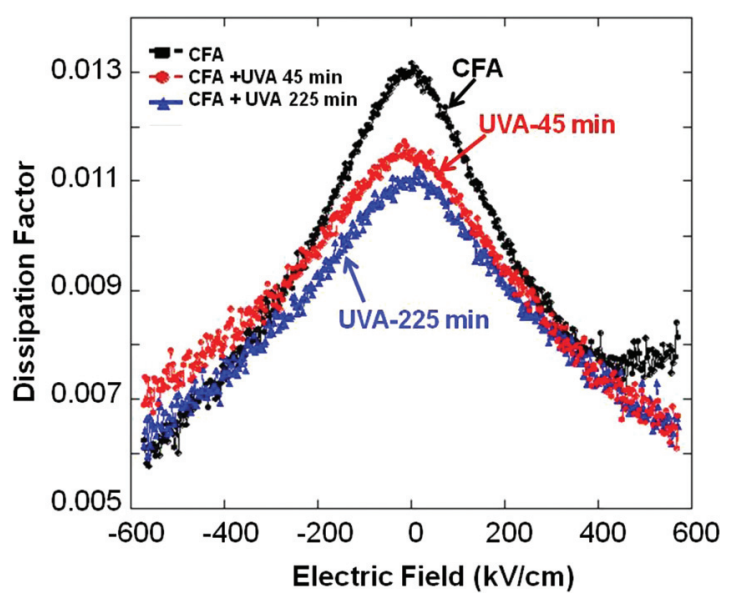

FIG. 37. The change of leakage current density as function of applied electric field for the CFA films (black) and CFA + UV-treated films for 45 (red) and $225 \mathrm{~min}$ (blue). Reprinted with permission from Cole et al., J. Appl. Phys. 110, 124105 (2011). Copyright 2011 American Institute of Physics.

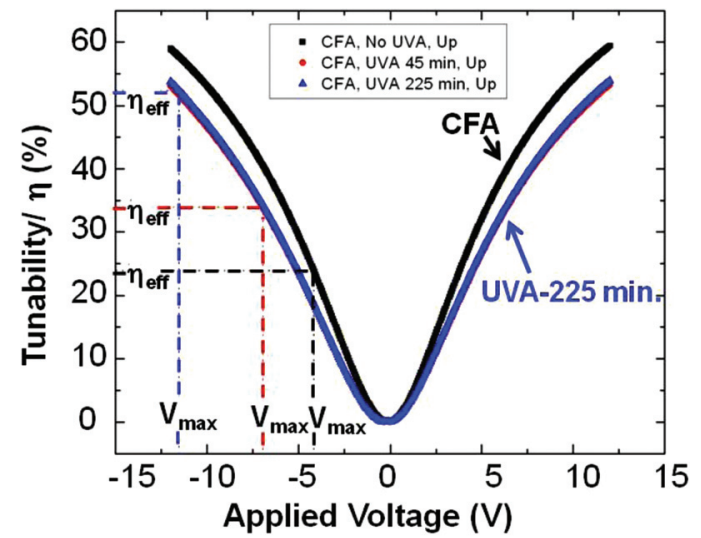

FIG. 38. Electric field dependence of tunability for the CFA (black) films, and the CFA + UV-treated films for 45 (red) and 225 (blue) min. $\mathrm{V}_{\max }$ represents the maximum applied bias which corresponds to the leakage current of $-500 \mathrm{pA}$, and $\eta_{\mathrm{eff}}$ represents the effective tunability corresponding to leakage current of $-500 \mathrm{pA}$. Reprinted with permission from Cole et al., J. Appl. Phys. 110, 124105 (2011). Copyright 2011 American Institute of Physics.

oxygen vacancy site. Long exposures ( $225 \mathrm{~min})$ allow a larger in-flux of oxygen into the films and further decrease oxygen vacancy defect density. The compensation of oxygen vacancies reduces the dielectric loss without degrading the dielectric tunability. Additionally, the UV-process science innovation was designed to be compatible with CMOS processing, hence promotes ease of scale-up and affordable component manufacturing.

\section{Reducing the conductor loss for varactor devices}

The conductor loss in the varactor devices need to be lower over the frequency range of interest, and this can be done by increasing the conductor thickness, which improves the conductivity of the electrodes, compared to thin electrodes $<1 \mu \mathrm{m}$. The increasing conductor thickness reduces the varactor device's parasitic series resistance and inductance. This approach should improve the conductor quality factor. Another approach to lowering the parasitic series resistance and inductances is by introducing an alternate design for the varactor, by combining two equal parallel varactors to give the original design value. Subramanyam et al. studied parallel capacitor architecture for varactors and concluded that parallel capacitor architecture improves the device Q. ${ }^{134}$ Figure 39 shows the parallel capacitor architecture implementation for microwave applications.

The benefits of the parallel capacitor architecture are:

(i) Reduced parasitic resistance and inductances due to the two parallel shunt lines to ground. This is the main reason for the improved Q.

(ii) Ability to handle higher DC voltages compared to a single shunt capacitor of larger overlap area. This can result in higher capacitance tuning ratio.

The only draw-back for this parallel capacitor architecture is the reduced shunt resistance as the two shunt resistors result in lower effective shunt resistance. This is the main reason that increasing the number of parallel capacitors beyond two results in lower Q compared to a single and dual varactors. 


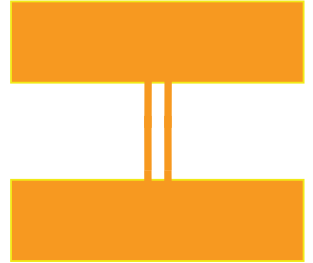

(a) Metal1 layer

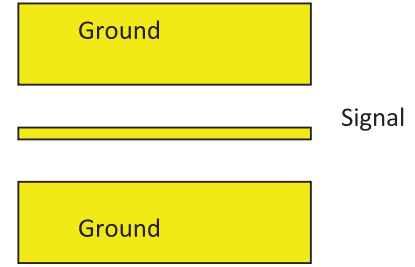

(b) Metal 2 layer

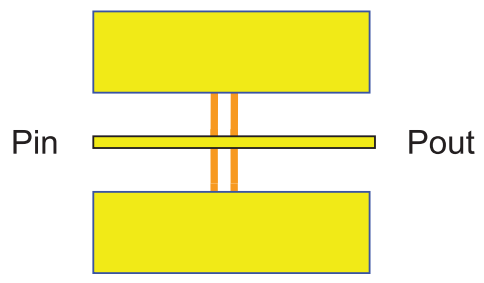

FIG. 39. Shunt varactor device structure with (a) Metal1 layer pattern showing the two shunt lines. (b) Metal2 layer pattern showing the coplanar waveguide transmission line (Ground, Signal, and Ground). (c) The top view of the two-capacitor varactor device with the overlapping area of the signal line and the shunt lines defining the two shunt capacitors. Reproduced by permission from Subramanyam et al., Integr. Ferroelectr. 112, 53 (2009). Copyright 2009 by Taylor \& Francis.

\section{Improving thermal stability in oxide thin films}

The development of a BST based thin film material that is temperature stable would serve to mitigate expensive packaging requirements and in addition, would enhance the device reliability, for stable performance as a function of temperature. BST thin films with extreme temperature insensitivity have been demonstrated recently, ${ }^{135-142}$ by conceiving a material design which controls the magnitude and the sign of the temperature coefficient of capacitance (TCC) of the BST thin film material in a parallel plate varactor device configuration. In particular, a very successful material design consisted of a compositionally stratified BST multi-layer heterostructure material design whereby the $\mathrm{Ba} / \mathrm{Sr}$ ratios range from $60 / 40$ to 90/10 within the thin film heterostructure. Such a BST thin film heterostructure can be considered as multiphase compositions with different phase transition temperatures (Curie temperature tailoring), where both a small TCC value, flat variation of permittivity within a wide temperature range, and high dielectric tunability and low dielectric loss could be obtained simultaneously. The phase shifters' temperature susceptibility was lowered by $70 \%$ with respect to the state-ofthe-art current phase shifter technology based on uniform composition BST materials. Specifically, the TCC for the compositionally stratified heterostructure was $\mathrm{TCC}_{20-90}$ $=-0.921 \mathrm{ppt}$ and a $\mathrm{TCC}_{20-(-10)}=-0.716$ ppt. ${ }^{135,137}$ The improvement of temperature susceptibility was also accompanied by significant improvements in dielectric tunability (device phase shift) and dissipation factor (device insertion loss); whereby the tunability was enhanced by $58 \%$ and insertion loss was improved by $50 \%$ with respect to uniform composition BST phase shifters (i.e., the compositionally stratified BST tunability was $65.5 \%$ at $444 \mathrm{kV} / \mathrm{cm}$ vs. Uniform BST tunability of $42 \%$ at $444 \mathrm{kV} / \mathrm{cm}$. The relatively lower dielectric loss tangent was explained by mobility of defects; i.e., it was suggested that the defects within the compositionally graded thin film were trapped at the interfaces to compensate for the polarization difference between the layers, hence resulted in improved dielectric loss.

In addition to developing this temperature stable phase shifter device, the results of this effort also served to ensure phase shifter device affordability by utilizing film fabrication and integration technology protocols that are industry standard, i.e., compliant with current CMOS process science methods and materials. Similar studies of compositionally graded thin films with different composites of BST and using different deposition techniques, such as PLD ${ }^{140}$ and RF sputtering, ${ }^{141}$ are also available in the open literature.

Another clever approach to obtaining temperature stabilization of BST varactors was proposed by Gevorgian et al. ${ }^{142}$ The fundamental concept centers on a capacitor which is composed of two ferroelectrics with different Curie temperatures. One of the ferroelectrics is in a paraelectric phase, while the other is in the ferroelectric state in the temperature interval between $T_{1}$ and $T_{2}$ (Fig. 40). In the temperature interval between the peaks, the permittivity of the ferroelectric phase increases with increasing temperature, while the permittivity of the paraelectric phase decreases. In this capacitor, the two thin film materials are "connected in parallel," hence the decreased permittivity of the paraelectric phase is compensated by the increased permittivity of ferroelectric phase. This concept was experimentally validated using a co-planar capacitor/varactor composed of PLD fabricated epitaxial BST 25/75 and BST 70/25 thin films inter-layered with a $\mathrm{MgO}$ seed and a $\mathrm{MgO}$ barrier layer. Here, the lower "seed" layer serves as a strain mitigator and the middle $\mathrm{MgO}$ layer serves as a diffusion barrier to ensure that the two ferroelectric layers do not form intermediate phases via diffusion during synthesis. The experimental results (Figure 41) demonstrated that the capacitance was almost independent of temperature over a wide temperature interval $(120-300 \mathrm{~K})$ and the TCC was less than $2 \times 10^{-4}$ in the temperature range of $150-250 \mathrm{~K}$. However, it is noteworthy to mention that at temperatures above $300 \mathrm{~K}$ the capacitance was no longer temperature independent and increased dramatically which is a major drawback of this material design. On a positive note, due to the overlapping "tails" of the temperature dependencies of the permittivities of the top and bottom ferroelectric films, the tunability of such a
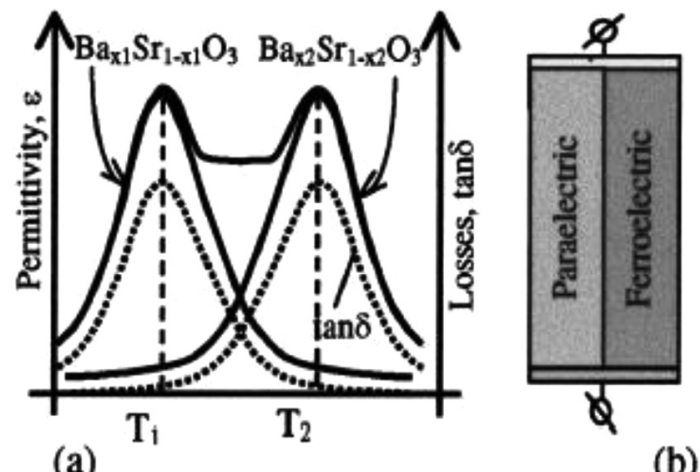

(b)

FIG. 40. Temperature dependencies of permittivity and loss (a) of a graded capacitor (b) the structure of the graded capacitor. Reprinted with permission from Gevorgian et al., Appl. Phys. Lett. 79, 1861 (2001). Copyright 2001 American Institute of Physics. 


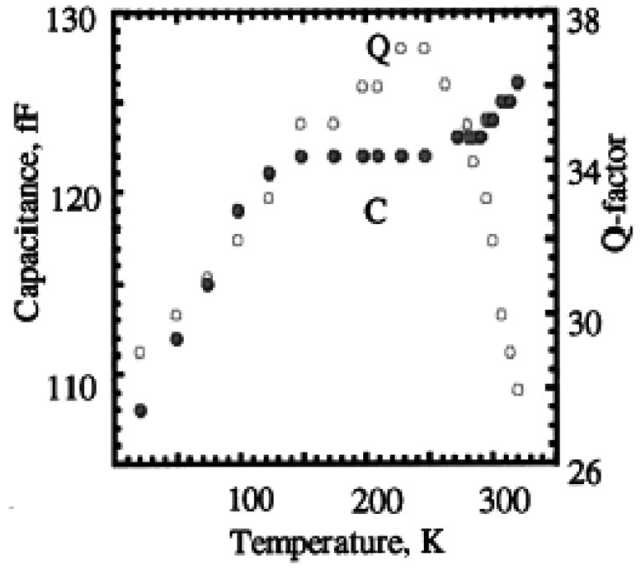

FIG. 41. Experimental dependencies of capacitance and quality factor at 1.0 MHz. Reprinted with permission from Gevorgian et al., Appl. Phys. Lett. 79, 1861 (2001). Copyright 2001 American Institute of Physics.

varactor is expected to be larger than if the varactor was composed of only uniform composition BST 25/75 or BST $75 / 25$ films. Although the quality factor of the varactor was highest over the same temperature interval where the capacitance was temperature stable, the $1 \mathrm{MHz} Q$-value is somewhat low, $Q \sim 36$, $\tan \delta \sim 0.028$ (Q $\sim 10 / \tan \delta \sim 0.1$ at $10 \mathrm{GHz}$ ) with respect to that obtained for the graded films and multilayer quasi-graded films.

\section{Substrates and packaging considerations}

For all BST devices whether or not they are a temperature stable material design, it is critical to protect the film from external ambience such as dirt, water, and humidity. Therefore, it is critical to develop low cost packaging methods utilizing robust linear dielectrics for both passivation (protective layers) and electrical insulation, whereby candidate film materials would include nitrides $\left(\mathrm{SiN}_{x}\right.$, for example) oxides $\left(\mathrm{SiO}_{2}\right)$ and/or carbides. Along similar lines, increasing the tunability of the BST film, could also be achieved via innovative material designs such as the compositional stratified structures described above. Device fabrication utilizing BST-based thin films requires appropriate substrates for both mechanical support and device integration. For both affordability and performance, the appropriate substrate must have a low insertion loss, be large area (4-6 inch diameter), low cost, thermally stable, chemically resistant, structurally suitable with good mechanical integrity, and must not perturb the performance of the BST film, i.e., the CTE, stress state, and lattice parameter must be compliant with the overlying BST thin film. In the recent past, BST-based thin films for $\mathrm{RF} /$ microwave applications have been deposited on ceramic $\left(\mathrm{MgO}, \mathrm{LaAlO}_{3}, \mathrm{SrTiO}_{3}\right)$ and a variety of other designer substrates. ${ }^{141-148}$ Major drawbacks of these substrates are the high cost of the ceramic substrates and the fact that ceramic substrates, such as $\mathrm{MgO}, \mathrm{LaAlO}_{3}$, and $\mathrm{SrTiO}_{3}$, are only available in small size geometries (2 in. wafers) which are not suitable for large-area film deposition. Hence, utilizing a large area, industry standard widely available support wafer which is compliant with the BST film properties and deposition method will allow more die per wafer hence promoting a lower phase shifter device cost. Candidate substrates include c- and R-plane sapphire, high resistivity silicon (HRS), polycrystalline alumina and glass. Significant progress has been made on large area BST thin film process development on sapphire and high resistivity Si. ${ }^{9,39}$ While the BST thin film based varactors fabricated on sapphire substrates could be useful for microwave applications, integration of BST thin film on standard CMOS silicon will be attractive for technology insertion into commercial RF/wireless communication circuits. Fabrication of BST thin films on high resistivity Si has been done by many researchers. In most cases, the relative dielectric permittivity at zero volts is reduced to almost half the value of the ones on sapphire substrate, to about 500 . The dielectric tuning is relatively good, more than 3:1 tuning range on high resistivity $\mathrm{Si}$ compared to $4: 1$ obtained on sapphire substrates. ${ }^{6,14,39}$ The dielectric loss-tangent is almost doubled for the high resistivity $\mathrm{Si}$, meaning the quality factor will be degraded by a factor of 2. Also, the integration of BST thin film on low-resistivity Si (standard CMOS Si) degrades the dielectric properties some more, as the relative dielectric constant at zero-bias drops to below 500. The dielectric loss-tangent also increases to close to 0.1 at zero-bias, which is considerably high for microwave applications. For integration of BST on low-resistivity Si, shunt capacitor implementation is attractive, since the substrate can be an effective ground. The challenge in this case is to making sure that the bottom electrode is a good ohmic contact with the Si substrate. Metal stack of $(\mathrm{Ti} / \mathrm{Al} / \mathrm{Ni} / \mathrm{Au} / \mathrm{Pt})$ can be used for getting a good ohmic contact to Si. Yue et al. recently studied implementation of parallel plate varactors on $\mathrm{Si}$ for specific capacitance values of $0.8,1.2,1.6,2,2.4,2.8,3.2,3.6,4,4.4$, and $4.8 \mathrm{pF}^{149}$ These capacitors were implemented as single capacitors based on the dielectric properties of BST measured on Si. BST thin film of $0.4 \mu$ m thickness was used in this study. The dielectric permittivity of BST thin films on low resistivity $\mathrm{Si}$ is approximately 200 at $0 \mathrm{~V}$. Figure 42

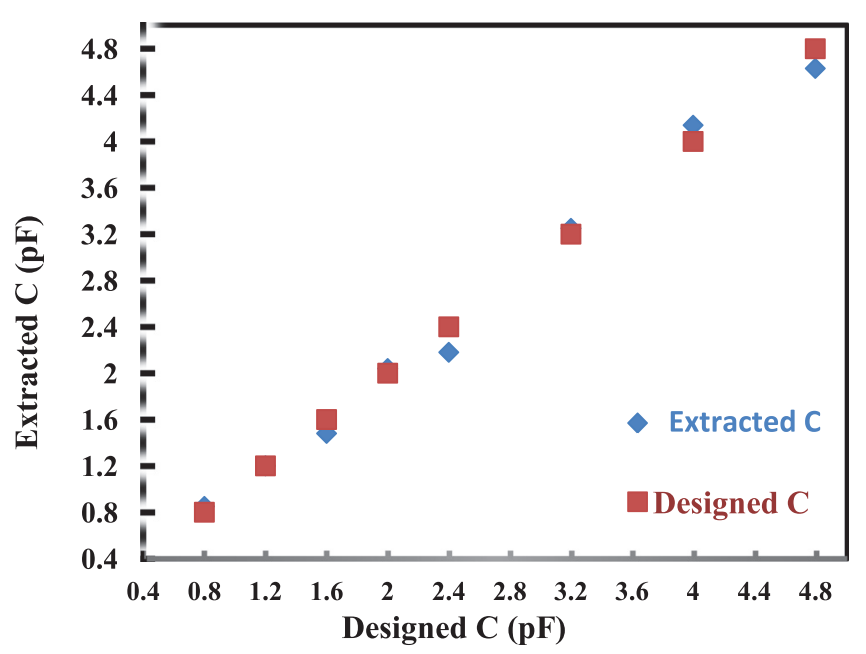

FIG. 42. Comparison of the extracted device capacitance at $0-\mathrm{V}$ to the design capacitance on low-resistivity CMOS Si. The extracted capacitance is obtained by matching the S-parameters of the measured devices to the Sparameters of the device equivalent circuit model. 
shows comparison of the zero-volt measured capacitance vs. designed capacitance.

The device capacitance was obtained by matching the measured S-parameters to the schematic circuit model as discussed in Sec. II. These varactors on low resistivity $\mathrm{Si}$ showed approximately 2:1 capacitance tuning with DC bias. Also, for integration with CMOS, a low temperature process will be necessary as typical physical vapor deposition processes need high temperatures above $750{ }^{\circ} \mathrm{C}$ for the BST thin films. It is clear that further work needs to be done on improving the BST varactor performance on low resistivity $\mathrm{Si}$.

\section{B. New techniques and heterostructures}

Currently, new technique for materials synthesis is required to enable design and fabrication of advanced materials with multi-functionalities and enhanced cross-response, e.g., mechanical, electrical, and magnetic field controlling polarization, magnetization, and strain. These multifunctional materials will exhibit a sensitive dependence of their physi$\mathrm{cal} / \mathrm{chemical}$ properties on the environments for various novel device developments. Therefore, design and fabrication of multifunctional thin films and heterostructures have become a key factor on the future device fabrications and integrations. Traditionally, multifunctional thin films materials can be fabricated by physical, chemical, or hybrid methods such as thermal vacuum deposition, electron/ion beam evaporation deposition, pulsed-laser deposition, dc/rf-sputtering, molecular beam epitaxy (including oxide MBE and MOMBE), metal-organic chemical vapor deposition, atomic layer deposition, sol gel, and polymer-assisted deposition. Various excellent results have been achieved from these advanced techniques. However, the key challenges on multifunctional complex oxide thin films and heterostructures are how to optimize the growth dynamics to achieve the designable physical properties, to control the microstructures and interfaces, to integrate multifunctional oxide materials on semiconductors, metals/alloys, carbon based structural materials, etc. Various efforts on the film/heterostructure fabrication and integration have been addressed in the past few years for their significant prospects in a wide range of applications. ${ }^{150-171}$ As a result of the extensive research in the area, a wide variety of oxide thin films and heterostructure such as ferromagnetic $\mathrm{SrRuO}_{3},{ }^{153,154}$ ferroelectric $\mathrm{Bi}_{4} \mathrm{Ti}_{3} \mathrm{O}_{12},{ }^{155-157}$ ferroelectric $(\mathrm{Ba}, \mathrm{Sr}) \mathrm{TiO}_{3},{ }^{158-162} \mathrm{Ba}(\mathrm{Ti}, \mathrm{Zr}) \mathrm{O}_{3},{ }^{163,164}$ (La,Ca) $\mathrm{MnO}_{3}$ (Ref. 165) thin films, giant dielectric constant $\mathrm{CaCu}_{3} \mathrm{Ti}_{4} \mathrm{O}_{12}$ thin films, ${ }^{166}$ and $\mathrm{PbTiO}_{3} / \mathrm{SrTiO} 3$ superlattices ${ }^{167}$ have been successfully achieved.

It has been well known that the interfacial structure and the lattice mismatch between the film and the substrate play a key role in controlling quality, microstructure and the physical properties of the heterostructures. For example, the lattice misfit can be used to enhance the Curie temperature of high temperature superconductors, ${ }^{151}$ to alter the Curie temperature of ferromagnetic transition in colossal magneto resistance materials, ${ }^{152}$ magnetic properties of ferrite films, ${ }^{172}$ and the dielectric properties in ferroelectric materials. ${ }^{173}$ Therefore, optimizing the growth conditions, both kinetically and dynamically, are the key issues on achieving the high quality complex oxide thin film epitaxy and heterostructure fabrication. Figure 43 shows the highly epitaxial complex oxide thin films with controllable microstructures where Figure 43(a) is the self-assembled oxide nano ribbon highly epitaxial structures and Figure 43(b) is the two sub layers $(\mathrm{La}, \mathrm{Ba}) \mathrm{CoO}_{3}-1$ and $(\mathrm{La}, \mathrm{Ba}) \mathrm{CoO}_{3}-2$ close to the interface in this heterostructure, which has a lattice mismatch of $-0.59 \%$ and $-1.31 \%$ with respect to the substrate. ${ }^{174}$ Self-patterned 3D nano structures with a dimension range from 2 to $10 \mathrm{~nm}$ were formed in the second layer (Figure 43(c)). These LBCO heterostructures with such 3D nanostructures show very interesting physical properties such as hard magnetism with an onset transition temperature near $190 \mathrm{~K}$, a large coercive field value, and a very large MR effect value (Fig. 44).

Furthermore, the physical properties of the interface engineered heterostructures can be tuned by designing the optimal multilayered combinations. Recently, interface engineered $\mathrm{BaTiO}_{3} / \mathrm{SrTiO}_{3}$ (Ref. 175) and $\mathrm{Mn}: \mathrm{Ba}(\mathrm{Ti}, \mathrm{Zr}) \mathrm{O}_{3} /$ $\mathrm{Mn}:(\mathrm{Ba}, \mathrm{Sr}) \mathrm{TiO}_{3}$ (Ref. 176) heterostructures were epitaxially grown on (001) $\mathrm{MgO}$ substrates by pulsed laser deposition. Microstructural characterizations by $\mathrm{x}$-ray diffraction and transmission electron microscopy indicate that the as-grown heterostructures are $c$-axis oriented with sharp interfaces. The in-plane interface relationships between the substrate and multilayered structures were determined to be $[001] \mathrm{SrTiO}_{3} /[001] \mathrm{BaTiO}_{3} /[001] \mathrm{MgO}$ and (100) $\mathrm{SrTiO}_{3} /$ (100) $\mathrm{BaTiO}_{3} /(100) \mathrm{MgO}$. The high-frequency microwave $(\sim 18 \mathrm{GHz})$ dielectric measurements reveal that the dielectric constant and dielectric loss of the nanolayered heterostructures are highly dependent upon the stacking period numbers and layer thicknesses. With the increase of the periodic number, or the decrease of each layer thickness, the dielectric constant dramatically increases and the dielectric loss tangent rapidly decreases, as seen in Figure 45.

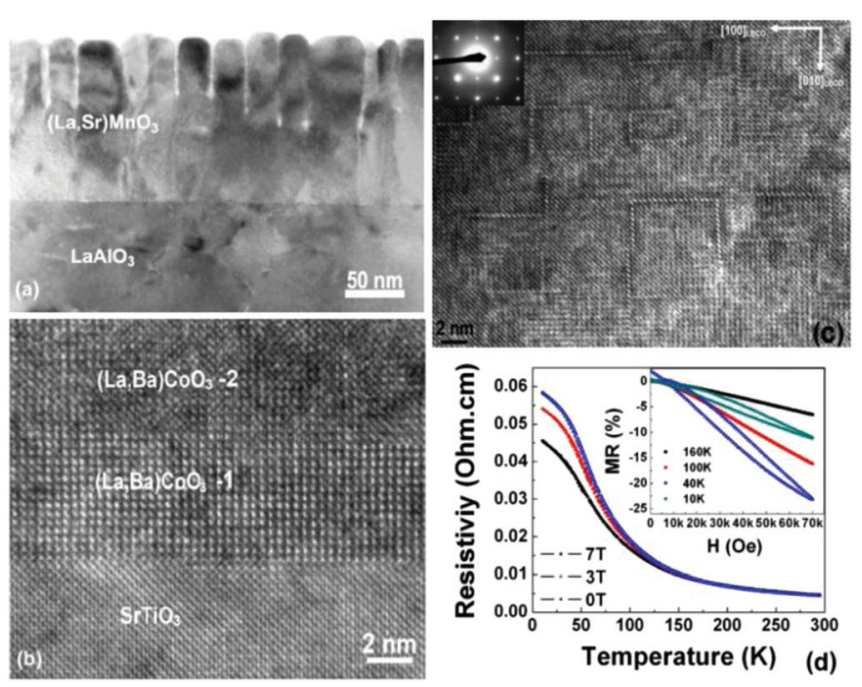

FIG. 43. (a) Self-assembled 1D LSMO nanorods, (b) atomic scale STO/LBCO interfaces, (c) atomic scale patterns in LBCO film, and (d) Tdependent electrical resistance and the magnetic-field-dependent MR behavior (inset) of the LBCO film. Reproduced by permission from He et al., Thin Solid Films 519, 4371 (2011). Copyright 2011 by Elsevier. 

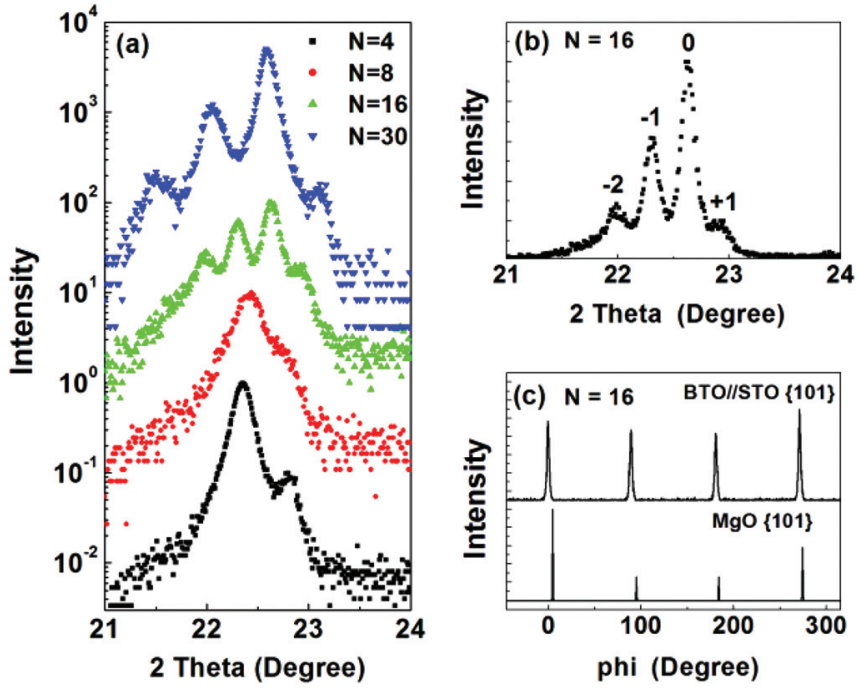

FIG. 44. A typical x-ray diffraction pattern of the as-grown BTO//STO multilayered thin films deposited on (001) $\mathrm{MgO}$ substrates, (a) with a total deposition time but different $\mathrm{N}$, (b) the $\mathrm{N}$ is 16 , (c) the $\phi$ scans taken along the $<101>$ direction of the multilayered thin films and $\mathrm{MgO}$ substrates, displaying that the films are epitaxial. Reproduced by permission from Liu et al., ACS Appl. Mater. Interfaces, 4(11), 5761 (2012). Copyright 2012 by American Chemical Society.

The strong interface effects were found when the combination period is larger than 16, or each STO layer is less than $6.0 \mathrm{~nm}$. The optimized dielectric performance was achieved with the best value for the loss tangent (0.02) and the dielectric constant (1320), which suggests that the BTO/STO heterostructures be promising for the development of the room temperature tunable microwave components. Interface engineering using multilayers of oxides have also resulted in low loss, highly tunable Ruddelsden-Popper phases $\mathrm{Sr}_{n+1} \mathrm{Ti}_{n} \mathrm{O}_{3 n+1}$. In these materials, in-plane crystallographic shear $(\mathrm{SrO})_{2}$ faults provide an alternative to point defects for accommodating non-stoichiometry. For $n=6$ phase, such strained films have a paraelectric to ferroelectric phase transition just below room temperature, providing high tunability at room temperature, at frequencies up to $125 \mathrm{GHz}$, with a high quality factor. ${ }^{168}$

Thermodynamically, various parameters, both macroscopically and microscopically or atomically, are strongly

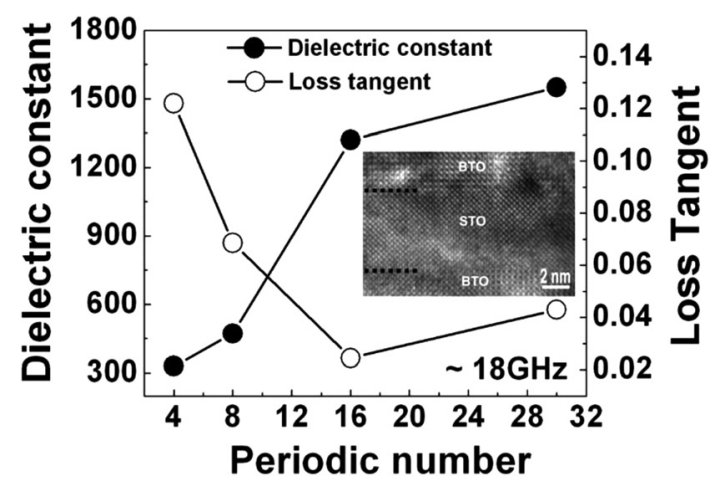

FIG. 45. The N dependent of the value of the dielectric constant and the loss tangent of the as-grown BTO//STO multilayered thin films on $\mathrm{MgO}$ substrates. The inset is the HRTEM image showing the multilayered structure of the heterostructures. Reproduced by permission from Liu et al., ACS Appl. Mater. Interfaces, 4(11), 5761 (2012). Copyright 2012 by American Chemical Society. affecting on the film growth such as temperature, pressure, interface structures and chemicals, beam energy, and bombardment rate. Kinetic-limit growth modeling studies indicate that the increase of the substrate temperature leads to the increase of the size and the energy barrier of the critical nucleus; the increase of the deposition rate will result in the reduction of the nucleation size and the decrease of the nucleation energy. Substrate surface is also another important parameter in governing the film growth. Low thermal budget will be significant in enhancing the thin film's electrical properties while minimizing defects, diffusion, and reliability issues. Thin film processing-structure-property correlation will be critical for new low thermal budget processing techniques such as laser and flash anneal type pulse processing techniques. Such techniques can provide necessary thermal budget within sub-ms compared to several minutes associated with conventional rapid thermal annealing (RTA) processes. Spark plasma sintering is another technique that allows for rapid synthesis at lower temperatures compared to conventional sintering and produces superior phase purity in polycrystalline composites. ${ }^{167}$

Epitaxial ferroelectric/ferromagnetic, dielectric, multiferroic, photo elastic, highly ionic conductive, and mixed conductive heterostructures may have important implications on the film behavior and offer a great opportunity to fabricate oxide nanostructures with desirable properties, and develop novel devices, such as sensitive nano conductivity sensors, solid state fuel cells, and electrochemical pumps and reactors, which are all important technological applications. These significant achievements of the interface engineered heterostructures enable us to develop new materials by fabricating novel 2D and/or 1D, two domain- and continuous composition-graded interface heterostructures, which may lay foundation for new materials research.

\section{Challenges for the multi-ferroic thin films}

The general key figures of merits for the voltage tunable $\mathrm{RF} /$ microwave multiferroic devices includes: tuning range, tuning speed, linearity, power handling, power consumption, size, weight, cost, etc., of which the sequence may vary depending on the applications. Key challenges of voltage tunable $\mathrm{RF} /$ microwave magnetic materials remain in the limited tunable range which is a direct consequence of the relatively weak magneto-electric coupling strength. The tuning range of demonstrated voltage tunable $\mathrm{RF} / \mathrm{microwave}$ multiferroic devices has been very limited, in the range of $1 \%-10 \%$ for tunable band pass band stop and band pass filters, ${ }^{26,27}$ even though multi-octave voltage tunable frequency range has been demonstrated in multiferroic heterostructures which can be directly applied in voltage tunable filters. ${ }^{26,27}$ At the same time, voltage tunable inductors with a tunable range of $5.5 / 1$ have been demonstrated. ${ }^{23}$ The full potential of voltage tunable $\mathrm{RF} /$ microwave multiferroic devices is yet to be realized.

Other broad challenges for achieving practical multiferroic RF devices are as follows. (i) Synthesis of 5-100 $\mu \mathrm{m}$ thick film yttrium iron garnet (YIG) or ferrites on piezoelectric substrates. Efforts to-date includes CVD growth of 
nickel ferrite on PMN-PT, but the losses are too high for device use. (ii) Direct growth techniques such as liquid phase epitaxy needs to be investigated for ferrite-piezoelectric heterostructures. (iii) Direct probing of interface strain for information on potential non-uniformity and effects on cross-coupling. (iv) Ferroelectric hysteresis and piezoelectric deformation (d) vs. voltage (V) are potential drawbacks for device losses and linearity of voltage tuning of operating frequency. Piezoelectrics with linear d vs. V have to be investigated. Although the d-values are much smaller compared to PZT or PMN-PT, tuning with ac voltage at resonance modes mode provide a path for desired tuning.

The key challenges in multiferroic antennas include achieving a relatively high permeability and low loss tangent across a broadband up to several $\mathrm{GHz}$ frequency range, cost of materials synthesis, and fabrication, etc. Conventional bulk magnetic materials typically show large magnetic loss tangent at $<1 \mathrm{GHz}$, which severely limits their applications in compact antennas at several GHz. Most recent spin spray deposited ferrite films with low loss tangent at $1-2 \mathrm{GHz},{ }^{176}$ which show great promise in compact antenna applications. ${ }^{177,178}$ However, achieving thick ferrite films with a thickness of $>20 \mu \mathrm{m}$ and a low loss tangent of $<5 \%$ remains an open challenge for the whole magnetics and multiferroics community.

\section{Challenges for modeling of complex oxide heterostructures}

For more than $60 \mathrm{yr}$, the classical Landau-GinzburgDevonshire (LGD) theory of weak first-order transitions has been extensively used in the study of physical phenomena in ferroic and multi-ferroic materials systems. ${ }^{179}$ In particular, the predictions and limitations of the conventional 2-4-6 Landau polynomial as applied to monocrystalline ferroelectric compounds have been investigated in detail and are well understood. ${ }^{180}$ The phenomenological theory provides a unified description of the changes in crystal form and of the variations of the coupled thermal, dielectric, and elastic properties that are observed on cooling through a sequence of ferroelectric phase transitions. The phenomenological theory is a useful tool for experimentalists because it provides a relatively simple framework within which the various physical properties of ferroelectric crystals of interest can be correlated. For example, the physical properties of a particular crystal as measured subject to one set of experimental conditions can be predicted subject to a different set of conditions, which may be inconvenient or otherwise difficult to access in the laboratory. Alternatively, the physical properties of a given crystal as measured by different types of experimental methods can be compared with the predictions of the theory and so checked against one another for consistency. In those instances where discrepancies between theory and experiment are observed, it is then possible to investigate the origins of the discrepancies, and to trace these discrepancies to factors such as the quality of the specimens under study, to errors in the measurements themselves, or more importantly, to the possible occurrence of other physical phenomena in the crystal that may not have been anticipated at the outset of the experiments. Following this approach, a self-consistent set of physical properties characterizing the mono-domain, single crystalline state can be established. For crystals for which such information is available, the polycrystalline-averaging method may then be used to estimate the properties of a corresponding randomly axed polycrystalline ceramic material. An introduction to the phenomenological theory as applied to ferroelectric compounds and solid solutions is given in Ref. 181 .

These LGD type phenomenological models have been successfully employed to describe the dielectric response and its tunability of monolithic ferroelectric thin films and multilayer heterostructures by taking into account electrical and mechanical boundary conditions of a thin film configuration. ${ }^{182,183}$ Of particular interest are the effects of internal stresses that result from the self-strain of the ferroelectric phase transformation, lattice-mismatch strains in epitaxial films and heterostructures, and thermal stresses that develop as the material is cooled down from the high processing temperatures to ambient temperature due to the thermal expansion coefficient difference between the multilayer film and the substrate. ${ }^{184,185}$

Theoretical studies based on such similar thermodynamic approaches to describe electrical interactions in multilayers and superlattices of ferroelectric materials indicate that when a ferroelectric and a paraelectric layer are brought together, there exists a critical paraelectric layer thickness at which the bilayer has a gigantic dielectric response due to the internal electric field that arises from the polarization mismatch between the layers (see Figure 46). ${ }^{186,187}$ Determination of this critical layer fraction allows the design of heterostructures with enhanced dielectric, pyroelectric, piezoelectric, and thermo-dielectric properties.

Using these theoretical results, several sets of experiments were conducted to determine the optimum parameters to obtain tunable active films with low loss and a temperature insensitive tunability over a temperature range of $100{ }^{\circ} \mathrm{C}{ }^{46,139}$ The parameters for the deposition were the deposition temperature, annealing temperature, film thickness, and the strength of the composition variations. Optimum conditions were found to exist in multilayered BST films deposited on Pt coated Si substrates via MOSD. The multilayer heterostructures consisted of three distinct layers of $\sim 220 \mathrm{~nm}$ nominal thickness with compositions corresponding to BST 63/37, BST 78/22, and BST 88/12. These multilayers exhibited a dielectric constant of 360 , dissipation factor of 0.012 , and a dielectric tunability of $65 \%$ at $444 \mathrm{kV} / \mathrm{cm}$ with minimal dispersion as a function of temperature ranging from 90 to $-10^{\circ} \mathrm{C}$ (Figure 47).

Utilizing the formalism described in Refs. 186 and 187, one can calculate the electric field dependent tunability of the BST multilayer heterostructures at different temperatures as a function of the external field. ${ }^{139}$ These results are plotted in Figure 48 and there is an excellent agreement with the theoretical predictions and the experimental results. This suggests that LGD-based theoretical formalisms can be used as a predictive tool to design tunable devices for telecommunications.

For many applications, solid solutions are the materials of choice because composition can be used as an external 
(a)

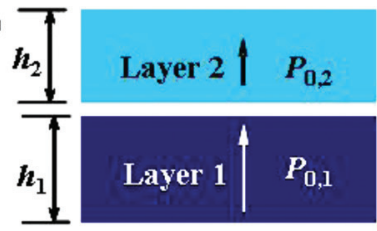

(b)

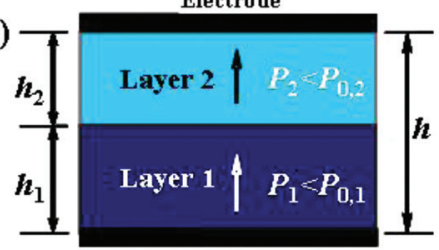

Electrode

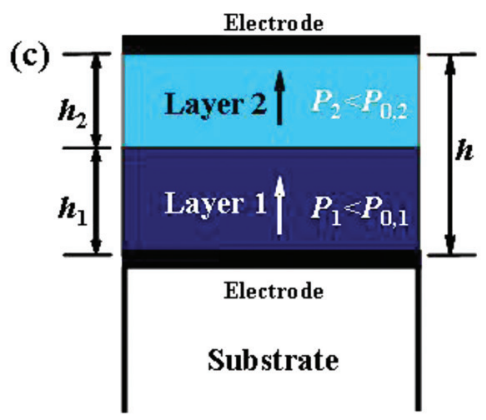

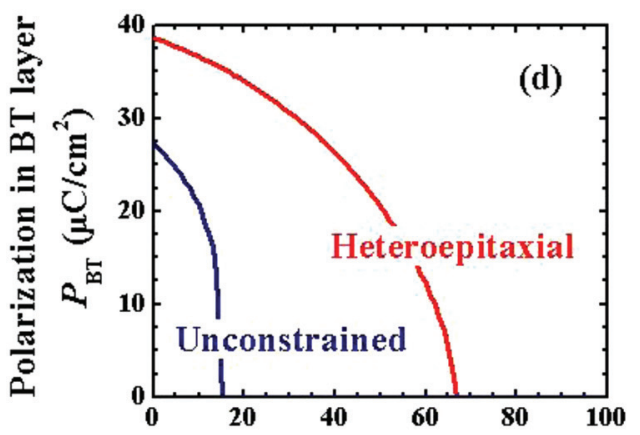

Volume fraction of ST, $\alpha_{\mathrm{ST}}(\%)$

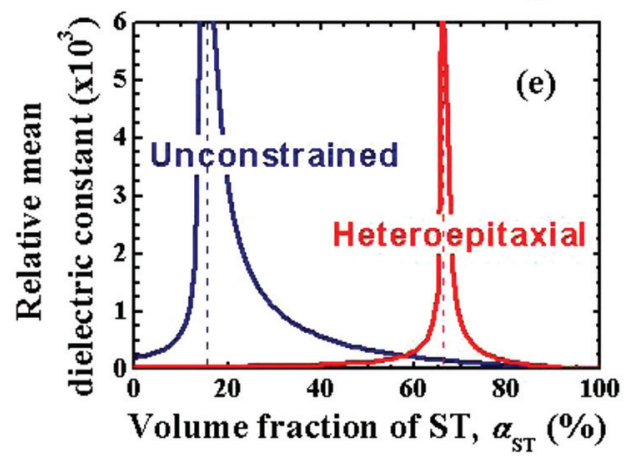

FIG. 46. (a) Two freestanding FE layers with initial polarizations $P_{0,1}$ and $P_{0,2}$. (b) A bilayer constructed by joining the layers in (a) with metallic top and bottom electrodes, (c) a heteroepitaxial bilayer on a thick substrate. Due to interlayer coupling the polarization in layer $1\left(P_{1}\right)$ is less than its uncoupled value $P_{0,1}$ and the polarization in layer $2\left(P_{2}\right)$ is larger than $P_{0,2}$, (d) theoretical polarization in an unconstrained and heteroepitaxial $\mathrm{BaTiO}_{3}-\mathrm{SrTiO}_{3}$ (BT-ST) bilayer, and (e) anomalous dielectric response of an unconstrained and heteroepitaxial BT-ST bilayer. Reprinted with permission from Roytburd et al., Appl. Phys. Lett. 87, 092902 (2005). Copyright 2005 American Institute of Physics. parameter to tune the properties of interest. The perovskite crystal structure admits a wide range of ion substitutions and there are numerous pseudo-binary ferroelectric systems showing complete solubility across the sub-solidus phase
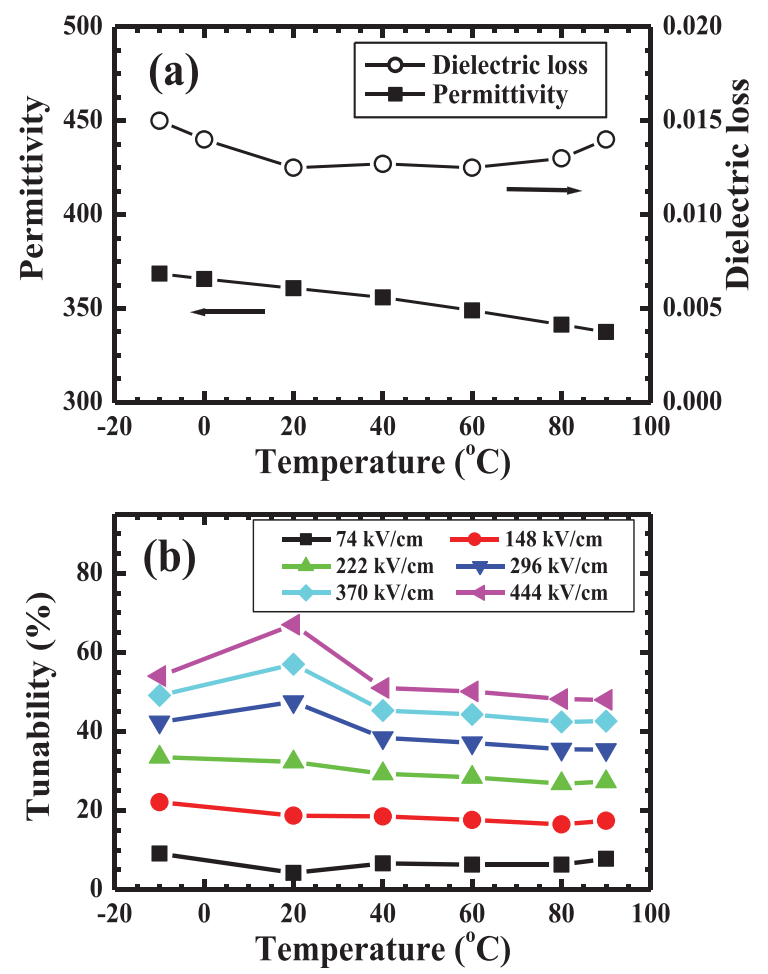

FIG. 47. (a) Dielectric permittivity and dielectric loss of BST multilayer films as a function of operation temperature; (b) temperature dependence of tunability at as a function of external electric field. Reprinted with permission from Zhong et al., Appl. Phys. Lett. 90, 092901 (2007). Copyright 2007 American Institute of Physics. diagram. The most interesting among these are those that have compounds of different symmetry located as opposing end members. In such systems, an isobaric change in composition leads to a change in Curie temperature and often to a change in the characteristic features of the paraelectric to

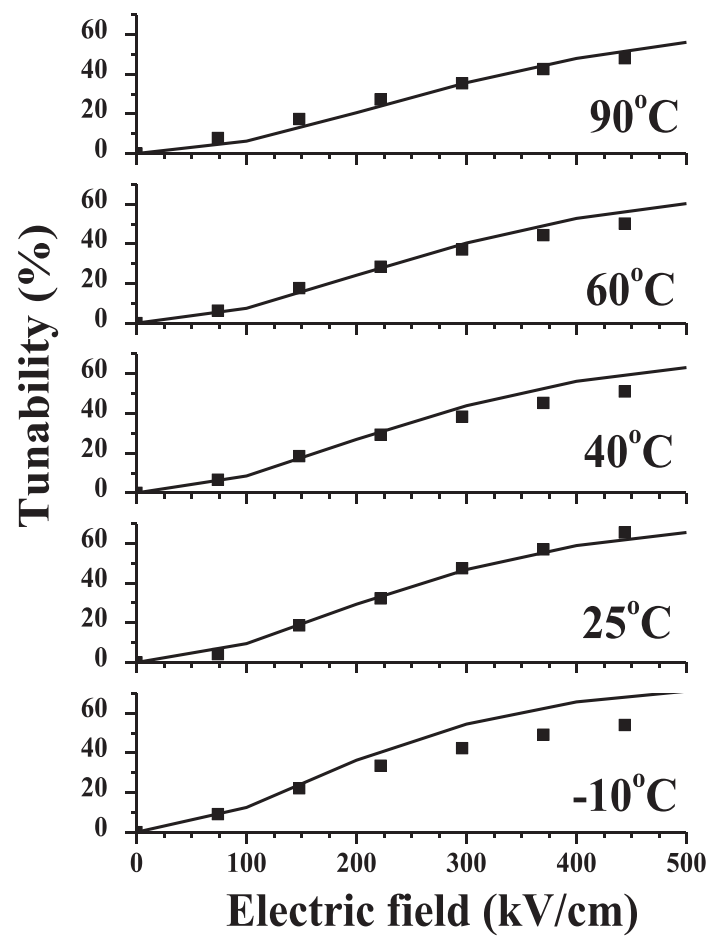

FIG. 48. Tunability of the BST multilayer as a function of external electric field at different temperatures (solid line: calculation results from theory; solid squares: experimental measurements). Reprinted with permission from Zhong et al., Appl. Phys. Lett. 90, 092901 (2007). Copyright 2007 American Institute of Physics. 
ferroelectric transition from first-order to second-order and/or to relaxor behavior. In the diffusionless compositiontemperature $(c-T)$ phase diagrams of these systems, singlephase regions of differing symmetry are separated by line boundaries. These boundaries may be crossed by either an isothermal change in composition or by a change in temperature at a fixed composition. In many instances, it is the extrema in physical properties that occur near these interferroelectric line boundaries that are exploited in device applications.

As a parameterized phenomenological theory, experimental data are required as inputs to adequately reproduce the behavior of specific ferroelectric materials. Somewhat surprisingly, for the ferroelectric compounds and solid solutions of technological importance, no universal parameterization scheme has been adopted. For example, the Landau polynomial as originally applied to ferroelectric barium titanate by Devonshire ${ }^{188,189}$ has been elaborated on many times, and several alternative expressions for the free energy have been reported that reproduce experimental data with varying degrees of agreement. ${ }^{190-194}$ The situation for ferroelectric solid solutions is more complicated still, because the dependence of the theory parameters on composition must also be explicitly determined. ${ }^{195,196}$ Approaches for doing so that utilize analytical parameterization schemes have recently been reported. ${ }^{197-200}$ An example of this approach is shown in Figure 49, where the composition-temperature phase diagrams of BST is compared with the experimental phase diagram. ${ }^{199}$ The figure indicates the location of two tricritical points (TCPs), and of the triple point (TP), where the cubic, tetragonal and rhombohedral phases are in equilibrium. The curving of the line of paraelectric to ferroelectric transitions corresponds to the temperature range where quantum saturation (QS) effects become important.

Predicting the behavior of ferroic and multi-ferroic materials, however, also involves phenomena that are relevant at

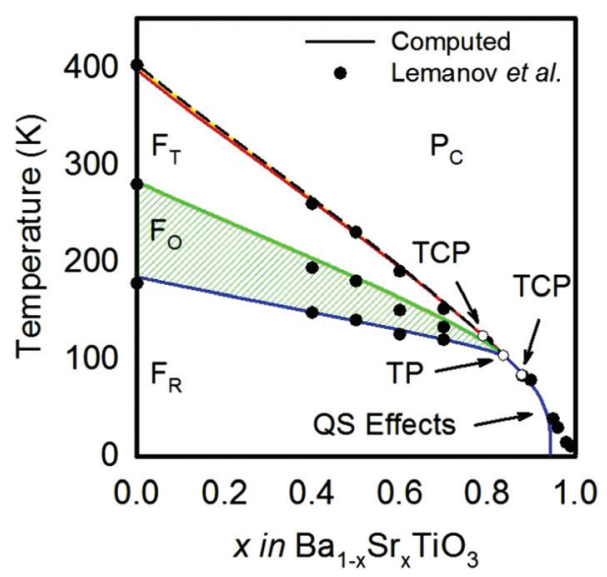

FIG. 49. Computed phase diagrams of BST in the composition-temperature plane compared with experimental data (solid circles). $\mathrm{P}_{\mathrm{C}}$ and $\mathrm{F}_{\mathrm{T}}, \mathrm{F}_{\mathrm{O}}$, and $\mathrm{F}_{\mathrm{R}}$ denote the paraelectric cubic and ferroelectric tetragonal, orthorhombic, and rhombohedral phases, respectively. The locations of two TCPs and the TP where $P_{C}, F_{T}$, and $F_{R}$ phases coexist are indicated by the open circles. The temperature range where QS effects become important is indicated by the curved line. Reproduced by permission from A. A. Heitmann and G. A. Rossetti, Jr., Integr. Ferroelectr. 126, 155-165 (2011). Copyright 2011 by Taylor \& Francis. length scales other than that of monodomain single crystals: the macro scale behavior is inherently a multiscale problem. The macro scale behavior arises from multiple contributions associated with the complicated sub-structure of these materials. For example, contributions to the dielectric response involve a distortion of the unit cell and the cooperative motion of domain walls. The optimization of dielectric materials for particular applications is often accomplished through the control of composition and through the addition of dopants that either enhance (donor) or pin (acceptor) domain wall motion. In order to link materials composition, dopant type and concentration, and dielectric properties, modeling efforts must address the material substructure across multiple scales of length and time and be capable of determining phase and domain stability under external forces. The challenges of multiscale modeling are depicted in Figure 50. Ab-initio computations and molecular dynamics simulations provide information on intrinsic material behavior at the unit cell level. The phenomenological models described above provide energy functions that reproduce phase transitions and can predict polarization reorientation processes under changes in external variables (temperature, composition, stress, or electric field). Micromechanical models introduce a grain orientation function and use volume averaging of the grain behavior to obtain the macro scale behavior. Phase field models provide a link between the energy functions of the phenomenological theory and domain structure and its evolution. At the higher level of structures and devices, finite element models are employed to capture interactions occurring at larger length scales.

Oxide thin film and multiferroic devices have inhomogeneous internal microstructural features at nano/micro scale and sometimes complex geometries, and hence they are beyond the reach of direct density function theory (DFT) calculations. On the other hand, conventional continuum modeling typically ignores the possible presence of domain structures and defects, and thus, may not be sufficient to address the effect of microstructure inhomogeneity on device performance. During the last ten years, phase-field method has emerged as a powerful computational tool to modeling phase transitions, domain structures, domain switching, and magneto electric couplings in ferroelectric/multiferroic thin films ${ }^{201-203}$ and heterostructures. ${ }^{204}$ Phase-field method describes a microstructure using a set of inhomogeneous order parameters and incorporates the fundamental thermodynamics of ferroic phase transitions as well as mesoscale theories of elasticity, electrostatics and magneto statics. For example, phase-field method has been applied to modeling the converse ME switching of magnetization in nanoscale layered heterostructures. ${ }^{205,206}$ It allows one to obtain the electric field induced changes in both the macroscopic magnetization and magnetic domain structures. Phase-field simulations have the potential to guide the design of devices. A Strain-Mediated Magneto resistive Random Access Memory $\left(\right.$ SM-MRAM) ${ }^{206}$ was designed through phase-field simulations, which is predicted to possess ultrahigh storage capacity, ultralow power dissipation, and room-temperature high-speed operation simultaneously, thus, offering a potentially significant improvement over existing MRAM 


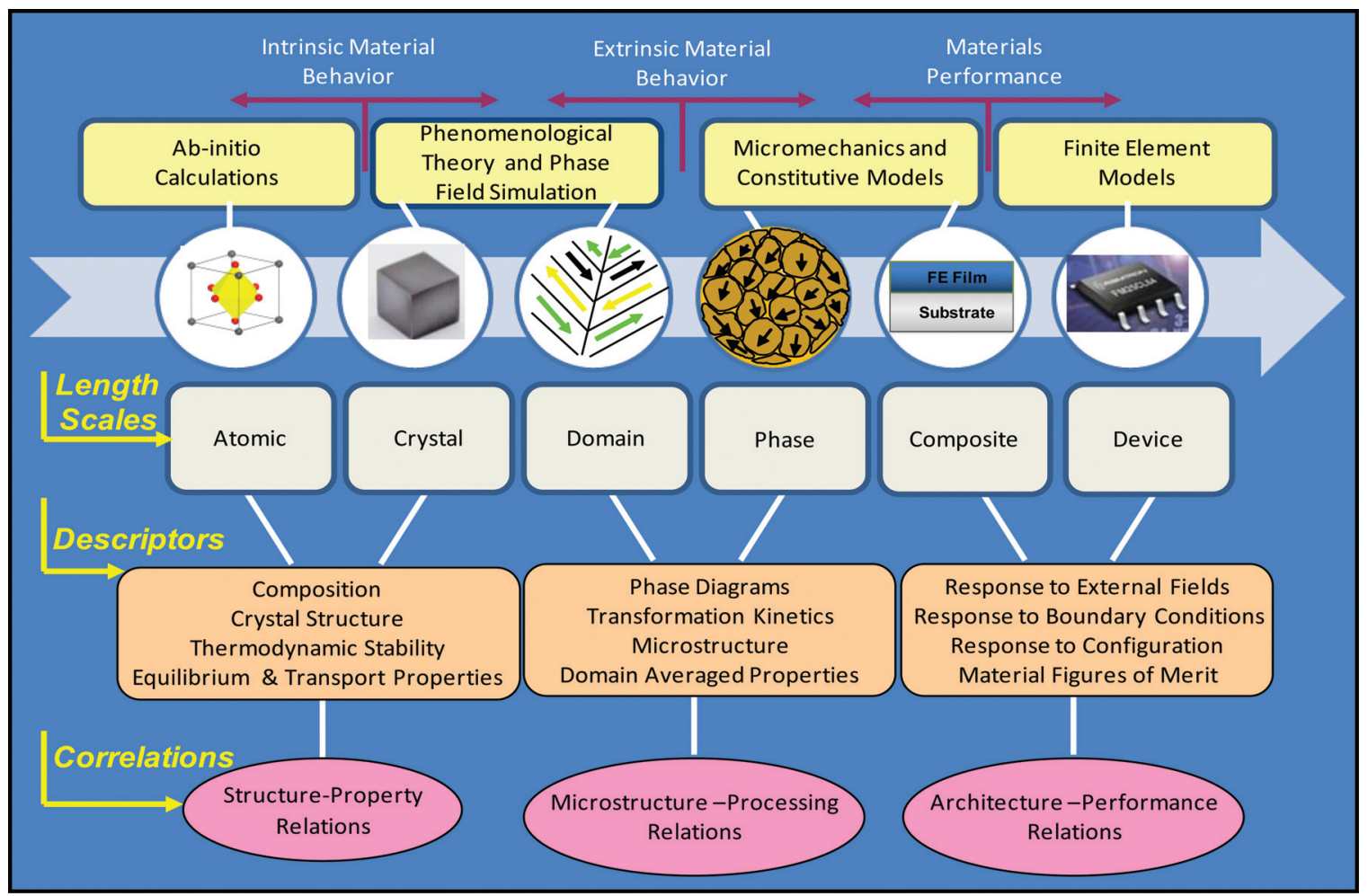

FIG. 50. Multi-scale modeling approaches for ferroic and multi-ferroic materials requires addressing phenomena across several characteristic length and time scales.

technologies or existing device concepts. However, there are several challenges that need to be overcome in order to make phase-field simulation a routine tool to fundamentally understand the basic mechanisms underlying the multifunctionality of heterostructures and to assist or guide the design of devices. First of all, phase-field simulations require input information for thermodynamic and kinetic parameters such as domain wall energy and domain wall mobility as well as structural and physical properties such as lattice parameters and elastic constants. While some of the data are available from experiments, DFT calculations are necessary to obtain properties which are otherwise not available experimentally. Therefore, a future direction along this line is to link firstprinciples calculations for structure and properties and the phase-field method of mesoscale domain structure evolution. Another challenge is the disparity of physical size of a device and the spatial scale of internal domain structure, which will make it difficult to spatially resolve the domain walls while attempting to model the performance of the whole device. Therefore, multiscale approaches are needed in such cases. The complex geometries of a device also pose a challenge to phase-field modeling. For many applications, boundary conditions with flat surfaces are adequate, e.g., the effective response of a thin film. In such cases, a superposition method can be applied to modeling domain structures in ferroelectric and multiferroic thin films ${ }^{207}$ which has an efficiency close to the 3D bulk systems with periodic boundary conditions. Another approach is to use Fast Fourier Transforms along the in-plane directions of a film and Chebyshev transforms along the out-of-plane direction. For non-flat surfaces, i.e., surfaces with arbitrary topologies, smooth boundary method ${ }^{208}$ or the more common finite element based numerical methods could be used to solve the multiphysics problems with appropriate boundary conditions on complex geometries. As described above, the phase-field method is a mature methodology based on fundamental physics. Therefore, recent efforts on physics-based design of devices with complex geometries have also focused on developing computational methods as design tools. ${ }^{209,210}$ Some important device geometries of interest include settings with partially electroded surfaces, ${ }^{211}$ or with surface features such as notches, islands, and surface tips. ${ }^{212,213}$ In the former case, the partially electrode surfaces lead to a problem posed on all-space because of existence of stray fields outside the device. In the latter case, a highly resolved calculation is required near regions where geometry and/or electric field changes abruptly, but a coarse discretization can be used away from these regions.

The computational methods for the settings described in the paragraph above include the use of the finite element method (FEM) to obtain adaptive discretizations that are fine in regions where complex microstructure forms, and coarsens further away. This enables the study of large computational domains at reasonable computational cost. In addition, complex geometries, such as notches and islands, are readily handled by FEM. Another important challenge is the allspace stray fields outside the device in combination with the complex boundary conditions posed by partial electrodes. Dirichlet-to-Neumann maps in combination with the boundary element method provide a method to handle these challenges at reasonable computational expense. ${ }^{214}$ Finally, modeling of space charge transport involving diffusion of 
dopants, role of oxygen vacancies, on the functionality of the thin films is another area of importance as well as of challenge. A recently formulated iterative version of this numerical method ${ }^{215}$ has enabled the study of space charges at complex domain patterns near the free surface of thin-film ferroelectrics.

\section{SUMMARY AND CONCLUSIONS}

This manuscript is an attempt to bridge the gap between materials researchers and product/system engineers with the technology needs. It is clear that multifunctional oxides and multiferroics have tremendous potential for applications in voltage tunable RF/microwave components. Significant progress has been made in understanding the properties and applications for ferroic oxide materials. Current state of the art BST thin film varactors could meet the requirements for decoupling capacitors, tunable filters, phase shifters, agile antennas, impedance matching networks/auto-tuners, and VCOs. The oxide thin film varactor technology has excellent potential to reduce the size, weight, cost, and improve functionality for the next generation communication systems. Areas for further BST varactor technology improvement include; reducing loss, improving temperature stability and improving integration capabilities with other silicon electronics. Technology development to manufacture devices utilizing multifunctional oxides on large scale should be one of the key research areas for timely and economic implementation into military systems and commercial products. New areas of technology research include materials with combinations of ferroic properties, known as multiferroic or multifunctional oxide materials which exhibit coupling of magnetic and electrostrictive properties, enabling electric field tuning of magnetic properties. Future studies could include combination of oxide and multi-ferroic thin films, heterostructures of oxide thin films for new device architectures based on these technologies.

\section{ACKNOWLEDGMENTS}

This manuscript is an outcome from the 2012 Workshop on Multifunctional Oxides sponsored by Army Research Laboratory (ARL) and the Army Research Office (ARO). The authors acknowledge the contributions of attendees of the workshop, including Professor Greg Carman (UCLA), Professor Stefan Zollner (New Mexico State), Professor Juan Nino (Florida), Professor Jacob Jones (Florida), Professor Mingzhong Wu (Colorado State), Dr. Kos Galatsis (Aneeve Tech), Dr. Pooran Joshi (Oak Ridge National Lab), Dr. Chakrapani Varanasi (ARO), and Dr. Mark Ulrich (ARO). Special thanks to Dustin Brown, University of Dayton, Ph.D. student for some of the redrawn art work.

${ }^{1}$ P. Cruz, N. B. Carvalho, and K. A. Remley, IEEE Microw. Mag. 11, 83 (2010)

${ }^{2}$ G. H. Haertling, J. Vacuum Sci. and Tech. A. 9, 414 (1991).

${ }^{3}$ A. K. Tagantsev, V. O. Sherman, K. F. Astafiev, J. Venkatesh, and N. Setter, J. Electroceram. 11, 5 (2003).

${ }^{4}$ A. Vorobiev, P. Rundqvist, K. Khamchane, and S. Gevorgian, Appl. Phys. Lett. 83, 3144 (2003).
${ }^{5}$ G. Subramanyam, F. Ahamed, and R. Biggers, IEEE Microw. Wirel. Compon. Lett. 15, 739 (2005).

${ }^{6}$ G. Subramanyam, F. Ahamed, R. Biggers, R. Neidhard, E. Nykiel, J. Ebel, R. Strawser, K. Stamper, and M. Calcatera, Microwave Opt. Technol. Lett. 47(4), 370 (2005).

${ }^{7}$ A. Kozyrev, A. Ivanov, T. Samoilova, O. Soldatenkov, K. Astafiev, and L. Sengupta, J. Appl. Phys. 88, 5334 (2000).

${ }^{8}$ J. Nath, D. Ghosh, J. P. Maria, A. I. Kingon, W. Fathelbab, P. D. Franzon, and M. B. Steer, IEEE Trans. MTT 53, 2707 (2005).

${ }^{9}$ B. Acikel, T. R. Taylor, P. J. Hansen, J. S. Speck, and R. A. York, IEEE Microw. Wirel. Compon. Lett. 12, 237 (2002).

${ }^{10}$ D. Kuylenstierna, A. Vorobiev, P. Linnér, and S. Gevorgian, IEEE Trans. Microwave Theory Tech. 53, 2164 (2005).

${ }^{11}$ A. Jamil, T. S. Kalkur, and N. Cramer, IEEE Trans. Ultrason. Ferroelectr. Freq. Control 54, 222 (2007).

${ }^{12}$ H. Jiang, M. Patterson, K. Pan, C. Zhang, G. Subramanyam, D. Kuhl, K. Leedy, and C. Cerny, IEEE Trans. Antennas and Propag. 60, 3111 (2012).

${ }^{13} \mathrm{~B}$. Acikel, "High performance barium strontium titanate varactor technology for low cost circuit applications," Ph.D. Dissertation (University of California at Santa Barbara, 2002).

${ }^{14}$ G. Subramanyam, M. Patterson, K. Leedy, R. Neidhard, C. Zhang, and G. Steinhauer, IEEE Trans. Ultrason. Ferroelectr. Freq. Control 57, 1692 (2010).

${ }^{15}$ O. G. Vendik, I. B. Vendik, and V. O. Sherman, Integ. Ferroelectr. 43, 81 (2002).

${ }^{16}$ M. Fiebig, J. Phys. D: Appl. Phys. 38, R123 (2005).

${ }^{17}$ N. Spaldin and M. Fiebig, Science 309, 391 (2005).

${ }^{18}$ W. Eerenstein, N. D. Mathur, and J. F. Scott, Nature 442, 759 (2006).

${ }^{19}$ C. W. Nan, M. I. Bichurin, S. X. Dong, D. Viehland, and G. Srinivasan, J. Appl. Phys. 103, 031101 (2008).

${ }^{20}$ J. Zhai, Z. Xing, S. X. Dong, J. F. Li, and D. Viehland, Appl. Phys. Lett. 88, 062510 (2006).

${ }^{21}$ J. F. Scott, Nature Mater. 6, 256 (2007).

${ }^{22}$ C. Israel, N. D. Mathur, and J. F. Scott, Nature Mater. 7, 93 (2008).

${ }^{23}$ J. Lou, D. Reed, M. Liu, and N. X. Sun, Appl. Phys. Lett. 94, 112508 (2009).

${ }^{24}$ Y. K. Fetisov and G. Srinivasan, Appl. Phys. Lett. 88, 143503 (2006).

${ }^{25}$ A. Ustinov, G. Srinivasan, and B. A. Kalinikos, Appl. Phys. Lett. 90, 031913 (2007).

${ }^{26}$ C. Pettiford, S. Dasgupta, J. Lou, S. D. Yoon, and N. X. Sun, IEEE Trans. Magn. 43, 3343 (2007).

${ }^{27}$ A. S. Tatarenko, V. Gheevarughese, and G. Srinivasan, Electron. Lett. 42, 540 (2006).

${ }^{28}$ W. Eerensten, M. Wiora, J. L. Prieto, J. F. Scott, and N. D. Mathur, Nature Mater. 6, 348 (2007).

${ }^{29}$ M. Liu, O. Obi, J. Lou, S. Stoute, J. Y. Huang, Z. Cai, K. S. Ziemer, and N. X. Sun, Appl. Phys. Lett. 92, 152504 (2008).

${ }^{30}$ Y. Imanaka, T. Shioga, and J. D. Baniecki, Fujitsu Sci. Tech. J. 38, 22 (2002).

${ }^{31}$ A. Tombak, J.-P. Maria, F. T. Ayguavives, G. T. Stauf, A. I. Kingon, and A. Mortazawi, IEEE Trans. Microw. Theory Tech. 51, 462 (2003).

${ }^{32}$ F. A. Miranda, F. W. Van Keuls, R. R. Romanofsky, and G. Subramanyam, Integr. Ferroelectr. 22, 269 (1998).

${ }^{33}$ A. Kumar and S. G. Manavalan, Surf. Coat. Technol. 198, 406 (2005).

${ }^{34}$ D. M. Potrepka, S. Hirsch, M. W. Cole, W. D. Nothwang, S. Zhong, and S. P. Alpay, J. Appl. Phys. 99, 014108 (2006).

${ }^{35}$ W. K. Simon, E. K. Akdogan, A. Safaria, and J. A. Bellotti, Appl. Phys. Lett. 87, 082906 (2005).

${ }^{36}$ A. Srivastava, V. Craciun, J. M. Howard, and R. K. Singh, Appl. Phys. Lett. 75, 3002 (1999).

${ }^{37}$ L. L. Lopez, J. Portelles, J. M. Siqueiros, G. A. Hirata, and J. Mckittrick, Thin Solid Films 373, 49 (2000).

${ }^{38}$ See www.solmates.nl for Solutions in Material Science: SoleMateS.

${ }^{39}$ P. Varanasi, K. Leedy, D. Tomich, and G. Subramanyam, Thin Solid Films 517, 2878 (2009).

${ }^{40} \mathrm{~J}$. Im, O. Auciello, P. K. Baumann, S. K. Streiffer, and D. Y. Kaufman, Appl. Phys. Lett. 76, 625 (2000).

${ }^{41}$ G. K. Wehner, J. Vac. Sci. Technol. A 1, 487 (1983).

${ }^{42}$ R. A. York, A. S. Nagra, E. Erker, T. Taylor, P. Perisawamy, J. Speck, S. Streiffer, D. Kaufmann, and O. Auciello, in Presentation at ISAF Conference, Honolulu, Hawaii, August (2000).

${ }^{43}$ B. Acikel, P. J. Hansen, T. R. Taylor, A. S. Nagra, J. S. Speck, and R. A. York, J. Integr. Ferroelectr. 39, 291 (2001).

${ }^{44}$ N. K. Pervez, P. J. Hansen, and R. A. York, Appl. Phys. Lett. 85, 4451 (2004). 
${ }^{45}$ J. Fukushima, K. Kodaira, and T. Matsushita, J. Mater. Sci. 19, 595 (1984).

${ }^{46}$ P. C. Joshi and M. W. Cole, Appl. Phys. Lett. 77, 289 (2000).

${ }^{47}$ M. W. Cole, W. D. Nothwang, C. Hubbard, E. Ngo, and M. Ervin, J. Appl. Phys. 93, 9218 (2003).

${ }^{48}$ M. W. Cole, E. Ngo, S. Hirsch, J. D. Demaree, S. Zhong, and S. P. Alpay, J. Appl. Phys. 102, 034104 (2007).

${ }^{49}$ K. K. Li, G. H. Haertling, and W. Y. Howng, Integr. Ferroelectr. 3, 81 (1993).

${ }^{50}$ X. Guo, Y. K. Zuo, K. K. Li, Q. Chen, and H. Jiang, J. Mater. Res. 22, 2125 (2007)

${ }^{51}$ H. Jiang, W. Hu, and S. Liang, Integr. Ferroelectr. 28, 63 (2000).

${ }^{52}$ F. W. Van Keuls, C. H. Mueller, R. R. Romanofsky, J. D. Warner, F. A. Miranda, and H. Jiang, Report No. NASA/TM-2002-210906/REV1, 2002.

${ }^{53}$ O. Huang, A. Bandyopadhyay, and S. Bose, Mater. Sci. Eng., B 116, 19 (2005).

${ }^{54}$ Y. Chen, T. Sakai, T. Chen, S. D. Yoon, A. L. Geiler, C. Vittoria, and V. G. Harris, Appl. Phys. Lett. 88, 062516 (2006).

${ }^{55} \mathrm{M}$. Abe and Y. Tamaura, J. Appl. Phys. 55, 2614 (1984).

${ }^{56}$ D. G. Schlom, L. Q. Chen, X. Q. Pan, A. Schmehl, and M. A. Zurbuchen, J. Am. Ceram. Soc. 91, 2429 (2008).

${ }^{57}$ K. Endo, S. Saya, S. Misawa, and S. Yoshida, Thin Solid Films 206, 143 (1991).

${ }^{58}$ L. L. H. King, K. Y. Hsieh, D. Lichterwalner, and A. I. Kingon, Appl. Phys. Lett. 59, 3045 (1991).

${ }^{59}$ B. Jalan, R. Engel-Herbert, N. J. Wright, and S. Stemmer, J. Vac. Sci. Technol. A 27, 461 (2009).

${ }^{60}$ B. Jalan, R. Engel-Herbert, J. Cagnon, and S. Stemmer, J. Vac. Sci. Technol. A 27, 230 (2009).

${ }^{61}$ E. Mikheev, A. P. Kajdos, A. J. Hauser, and S. Stemmer, Appl. Phys. Lett. 101, 252906 (2012).

${ }^{62}$ J. F. Scott, Annu. Rev. Mater. Sci. 28, 79 (1998).

${ }^{63}$ S. Ezhilvalavan and T.-Y. Tseng, Mater. Chem. Phys. 65, 227 (2000).

${ }^{64}$ W. G. Breiland and G. H. Evans, J. Electrochem. Soc. 138, 1806 (1991).

${ }^{65}$ G. S. Tompa, P. A. Zawadzki, K. Moy, M. McKee, A. G. Thompson, A. I. Gurary, E. Wolak, P. Esherick, W. G. Breiland, G. H. Evans, N. Bulitka, J. Hennessy, and C. J. L. Moore, J. Cryst. Growth 145(1-4), 655 (1994)

${ }^{66}$ F. Fitsilis, S. Regnery, P. Ehrhart, R. Waser, F. Schienle, M. Schumacher, M. Dauelsberg, P. Strzyzewski, and H. Juergensen, J. Eur. Ceram. Soc. 21, 1547 (2001)

${ }^{67}$ T. Li, P. A. Zawadzki, and R. A. Stall, Proc. SPIE 3214, 104 (1997).

${ }^{68}$ J.-H. Lee and S.-W. Rhee, J. Electrochem. Soc. 146, 3783 (1999).

${ }^{69}$ N. M. Sbrockey, M. W. Cole, T. S. Kalkur, M. Luong, J. E. Spanier, and G. S. Tompa, Integr. Ferroelectr. 126, 21 (2011).

${ }^{70}$ A. Tombak, IEEE Trans. Microwave Theory Tech. 55, 370 (2007).

${ }^{71}$ R. Senthilnathan and J. L. Prince, IEEE J. Solid-State Circuits 26, 1724 (1991).

${ }^{72} \mathrm{H}$. Li and G. Subramanyam, IEEE Trans. Ultrason. Ferroelectr. Freq. Control 55, 2552 (2008).

${ }^{73}$ T. S. Kalkur, N. Sbrockey, G. S. Tompa, M. W. Cole, and S. P. Alpay, Integr. Ferroelectr. 126, 28 (2011).

${ }^{74}$ T. S. Kalkur, N. M. Sbrockey, G. S. Tompa, P. Alpay, J. E. Spanier, E. M. Galow, and M. W. Cole, Integr. Ferroelectr. 112, 1 (2010).

${ }^{75} \mathrm{~S}$. Gevorgian and A. Vorobiev, in Proceedings of European Microwave Conference (2010), p. 1210.

${ }^{76}$ S. A. Sis, V. Lee, and A. Mortazawi, IEEE MTT-S Int. Microwave Symp. Dig. 1-4 (2011).

${ }^{77}$ A. Volatier, E. Defay, M. Aid, A. N'hari, P. Ancey, and B. Dubus, Appl. Phys. Lett. 92, 032906 (2008)

${ }^{78} \mathrm{~J}$. Berge, A. Vorobiev, W. Steichen, and S. Gevorgian, IEEE Microw. Wirel. Compon. Lett. 17, 655 (2007).

${ }^{79}$ G. N. Saddik, D. S. Boesch, S. Stemmer, and R. A. York, Appl. Phys. Lett. 91, 043501 (2007).

${ }^{80}$ T. S. Kalkur, N. Sbrockey, G. Tompa, and P. Alpay, in Proceedings of the IEEE Symposium on Applications of Ferroelectrics (ISAF) (2012), pp. 1-4.

${ }^{81}$ Y. Zhang and T. S. Kalkur, in Proceedings of Progress in Electromagnetics Research Symposium (2011), p. 999.

${ }^{82}$ K. Groves, M.S. Thesis, University of Dayton, 2007.

${ }^{83}$ L. Y. Chen, R. Forse, D. Chase, and R. A. York, IEEE MTT-S Int. Microwave Symp. Dig. 1, 261 (2004).

${ }^{84}$ T. S. Kalkur, C. Cotey, K. Chen, and S. Sun, Integr. Ferroelectr. 56, 1123 (2003)
${ }^{85}$ A. Victor, J. Nath, D. Gosh, B. B. Boyette, J. P. Maria, M. B. Steer, A. I. Kingon, and G. T. Stauf, IEE Proc. Microwaves, Antennas Propag. 153, 96 (2006).

${ }^{86}$ A. Kabir and T. S. Kalkur, IEEE Trans. Ultrason. Ferroelectr. Freq. Control 60, 1638 (2013)

${ }^{87}$ C. Kong, H. Li, S. Jiang, J. Zhou, X. Chen, and C. Chen, in Proceedings of International Radio Frequency Integration Technology (2011), p. 197.

${ }^{88}$ T. S. Kalkur and A. Kabir, Integr. Ferroelectr. 125, 36 (2011).

${ }^{89}$ M. W. Cole, P. C. Joshi, M. H. Ervin, M. C. Wood, and R. L. Pfeffer, Thin Solid Films 374, 34 (2000).

${ }^{90}$ K. Pervez, P. J. Hansen, and R. A. York, Appl. Phys. Lett. 85, 4451 (2004).

${ }^{91}$ F. W. Van Keuls, F. Miranda, R. Romanofsky, J. Warner, and S. Alterovitz, Appl. Phys. Lett. 71, 3075 (1997).

${ }^{92}$ R. Romanofsky, Proc. IEEE 95, 1968 (2007).

${ }^{93}$ J. Lou, R. E. Insignares, Z. Cai, K. S. Ziemer, M. Liu, and N. X. Sun, Appl. Phys. Lett. 91, 182504 (2007).

${ }^{94}$ M. Liu, J. Lou, and N. X. Sun, Adv. Funct. Mater. 21, 2593 (2011).

${ }^{95}$ M. Liu, J. Lou, M. Li, O. Obi, X. Xing, and N. X. Sun, J. Appl. Phys. 109, 07D913 (2011).

${ }^{96}$ M. Liu, O. Obi, Z. Cai, J. Lou, G. Yang, K. S. Ziemer, and N. X. Sun, J. Appl. Phys. 107, 073916 (2010).

${ }^{97}$ J. Lou, M. Liu, D. Reed, Y. Ren, and N. X. Sun, Adv. Mater. 21, 2536 (2009).

${ }^{98}$ M. Liu, O. Obi, J. Lou, Y. Chen, Z. Cai, S. Stoute, M. Espanol, M. Lew, X. Situ, K. S. Ziemer, V. G. Harris, and N. X. Sun, Adv. Funct. Mater. 19, 1826 (2009).

${ }^{99}$ M. Liu, O. Obi, J. Lou, S. Stoute, Z. Cai, K. S. Ziemer, and N. X. Sun, J. Phys. D: Appl. Phys. 42, 045007 (2009).

${ }^{100}$ J. Lou, D. Reed, C. Pettiford, M. Liu, P. Han, S. Dong, and N. X. Sun, Appl. Phys. Lett. 92, 262502 (2008).

${ }^{101}$ J. Lou, D. Reed, Y. H. Ren, and N. X. Sun, J. Appl. Phys. 109, 07D731 (2011).

${ }^{102}$ A. S. Tatarenko, V. Gheevarughese, G. Srinivasan, O. V. Antonenkov, and M. I. Bichurin, J. Electroceram. 24, 5 (2010).

${ }^{103}$ A. A. Semenov, S. F. Karmanenko, V. E. Demidov, B. A. Kalinikos, G. Srinivansan, A. N. Slavin, and J. V. Mantese, Appl. Phys. Lett. 88, 033503 (2006).

${ }^{104}$ N. X. Sun and G. Srinivasan, SPIN 02, 1240004 (2012).

${ }^{105}$ K. R. Smith, V. I. Vasyuchka, M. Wu, G. A. Melkov, and C. E. Patton, Phys. Rev. B 76, 054412 (2007).

${ }^{106}$ E. N. Beginin, S. V. Grishin, M. A. Morozova, and Yu. P. Sharaevsskii, Tech. Phys. Lett. 35, 853 (2009).

${ }^{107}$ C. Pettiford, S. Dasgupta, J. Lou, S. D. Yoon, and N. X. Sun, IEEE Trans Magn. 43, 3343 (2007).

${ }^{108}$ X. Yang, J. Wu, S. Beguhn, T. Nan, Y. Gao, Z. Zhou, and N. X. Sun, IEEE Micro. Wireless Comp. Lett. 23, 184 (2013).

${ }^{109}$ T. Nan, Y. Hui, M. Rinaldi, and N. X. Sun, Sci. Rep. 3, 1985 (2013).

${ }^{110}$ G. M. Yang, O. Obi, and N. X. Sun, Microwave Opt. Technol. Lett. 54, 230 (2012).

${ }^{111}$ G.-M. Yang, X. Xing, A. Daigle, O. Obi, M. Liu, J. Lou, S. Stoute, K. Naishadham, and N. X. Sun, IEEE Trans. Antennas Propag. 58, 648 (2010).

${ }^{112}$ G. M. Yang, X. Xing, A. Daigle, O. Obi, M. Liu, S. Stoute, K. Naishadham, and N. X. Sun, IET Proc. Microwaves, Antennas Propag. 4, 1172 (2010).

${ }^{113}$ G. M. Yang, A. Shrabstein, X. Xing, O. Obi, S. Stoute, M. Liu, J. Lou, and N. X. Sun, IEEE Trans. Magn. 45, 4191 (2009).

${ }^{114}$ G. M. Yang, X. Xing, A. Daigle, M. Liu, O. Obi, S. Stoute, K. Naishadham, and N. X. Sun, IEEE Trans. Antennas Propag. 57, 2190 (2009).

${ }^{115}$ G. M. Yang, X. Xing, A. Daigle, M. Liu, O. Obi, J. W. Wang, K. Naishadham, and N. X. Sun, IEEE Trans. Magn. 44, 3091 (2008).

${ }^{116}$ G. M. Yang, A. Daigle, M. Liu, O. Obi, S. Stoute, K. Naishadham, and N. X. Sun, Electron. Lett. 44, 332 (2008).

${ }^{117}$ M. W. Cole, E. Ngo, C. Hubbard, S. G. Hirsch, M. Ivill, W. L. Sarney, J. Zhang, and S. P. Alpay, J. Appl. Phys. 114, 080341 (2013).

${ }^{118}$ E. Lage, C. Kirchhof, V. Hrkac, L. Kienle, R. Jahns, R. Knöchel, E. Quandt, and D. Meyners, Nat. Mater. 11, 523 (2012).

${ }^{119}$ M. W. Cole, R. C. Toonen, S. G. Hirsch, E. Ngo, R. R. Romanofsky, F. Van Keuls, C. Hubbard, M. Ivill, and D. Demaree, Integrated Ferroelectrics 111, 68 (2009).

${ }^{120}$ R. Liedtke, S. Hoffmann, and R. Waser, J. Am. Ceram. Soc. 83, 436 (2000).

${ }^{121}$ A. S. Bhalla, R. Y. Gao, and R. Roy, Mater. Res. Innovations 4, 3 (2000). 
${ }^{122}$ B. Acikel, T. R. Taylor, P. J. Hansen, J. S. Speck, and R. A. York, IEEE MTT-S Int. Microwave Symp. Dig. 3, 1467 (2002).

${ }^{123}$ G. Subramanyam, M. Patterson, K. Leedy, R. Neidhard, C. Varanasi, C. Zhang, and G. Steinhauer, Integr. Ferroelectr. 112, 53 (2010).

${ }^{124}$ M. W. Cole and R. G. Geyer, Mech. Mater. 36, 1017 (2004).

${ }^{125}$ S. Y. Wang, B. L. Cheng, C. Wang, S. A. T. Redfern, S. Y. Dai, K. J. Jin, H. B. Lu, Y. L. Zhou, Z. H. Chen, and G. Z. Yang, J. Phys. D: Appl. Phys. 38, 2253 (2005).

${ }^{126}$ W. Chang, S. W. Kirchoefer, J. M. Pond, J. S. Horwitz, and L. Sengupta, J. Appl. Phys. 92, 1528 (2002).

${ }^{127}$ M. W. Cole, W. D. Nothwang, J. D. Demaree, and S. Hirsch, J. Appl. Phys. 98, 024507 (2005).

${ }^{128}$ A. S. Reshmia, P. S. Asha, M. K. Krishnaprasad, M. K. Jayaraj, and M. T. Sebastian, J. Alloys Compd. 509, 6561 (2011).

${ }^{129}$ J. W. Lu and S. Stemmer, Appl. Phys. Lett. 83, 2411 (2003).

${ }^{130}$ J. Park, J. W. Lu, S. Stemmer, and R. A. York, J. Appl. Phys. 97, 084110 (2005)

${ }^{131}$ A. Podpirka, M. W. Cole, and S. Ramanathan, Appl. Phys. Lett. 92, 212906 (2008).

${ }^{132}$ K.-H. Chen, C.-L. Wu, J.-Y. Lin, and C.-M. Cheng, Adv. Mater. Res. 239-242, 1002 (2011).

${ }^{133}$ M. W. Cole, R. C. Toonen, M. Ivill, S. G. Hirsch, E. Ngo, and C. Hubbard, J. Appl. Phys. 110, 124105 (2011).

${ }^{134}$ G. Subramanyam, M. Patterson, K. Leedy, R. Neidhard, C. Varanasi, and G. Steinhauer, Integr. Ferroelectr. 125, 11 (2011).

${ }^{135}$ M. W. Cole and S. P. Alpay, in Ferroelectrics - Material Aspects, edited by M. Lallart (InTech, 2011), p. 149, see http://www.intechopen.com/ articles/show/title/performance-enhanced-complex-oxide-thin-films-fortemperature-stable-tunable-device-applications-a-m.

${ }^{136}$ M. W. Cole, C. V. Weiss, E. Ngo et al., Appl. Phys. Lett. 92, 182906 (2008).

${ }^{137}$ M. W. Cole, R. G. Geyer, C. Hubbard, E. Ngo, M. Ervin, M. Wood, and W. Nothwang, Revista Mexicana de Fisica 50, 232 (2004).

${ }^{138}$ M. B. Okatan, M. W. Cole, and S. P. Alpay, J. Appl. Phys. 104, 104107 (2008).

${ }^{139}$ S. Zhong, S. P. Alpay, M. W. Cole et al., Appl. Phys. Lett. 90, 092901 (2007).

${ }^{140}$ X. Zhu, N. Chong, H. L. W. Chan, C. L. Choy, K. H. Wang, Z. Liu et al., Appl. Phys. Lett. 80, 3376 (2002).

${ }^{141}$ J. Sigman, P. G. Clem, C. D. Nordquist, J. J. Richardson, and J. T. Dawley, J. Appl. Phys. 102, 054106 (2007).

${ }^{142}$ S. Gevorgian, P. K. Petrov, Z. Ivanov, and E. Wikborg, Appl. Phys. Lett. 79, 1861 (2001).

${ }^{143}$ W. Chang, J. S. Horwitz, A. C. Carter, J. M. Pond, S. W. Kirchoefer, C. M. Gilmore, and D. B. Chrisey, Appl. Phys. Lett. 74, 1033 (1999).

${ }^{144}$ M. W. Cole, P. C. Joshi, M. Ervin, M. Wood, and R. L. Pfeiffer, J. Appl. Phys. 92, 3967 (2002).

${ }^{145}$ S. B. Qadri, J. S. Horwitz, D. B. Chrisey, R. C. Y. Auyeung, and K. S. Grabowski, Appl. Phys. Lett. 66, 1605 (1995).

${ }^{146}$ W. J. Kim, W. Chang, S. B. Qadri, J. M. Pond, S. W. Kirchorfer, D. B. Chrisey, and J. S. Horwitz, Appl. Phys. Lett. 76, 1185 (2000).

${ }^{147}$ E. J. Cukauskas, S. W. Kirchoefer, and J. M. Pond, J. Appl. Phys. 88, 2830 (2000).

${ }^{148}$ Y. Gim, T. Hudson, Y. Fan, C. Kwon, A. T. Findikoglu, B. J. Gibbons, B. H. Park, and Q. X. Jia, Appl. Phys. Lett. 77, 1200 (2000).

${ }^{149}$ H. Yue, D. Brown, G. Subramanyam, K. Leedy, and C. Cerny, paper presentation in the 2013 IEEE International Symposium on Applications of Ferroelectrics (ISAF 2013).

${ }^{150}$ H. Koinuma, "Why crystal engineering of oxides," MRS Bulletin 19, 21-22 (1994).

${ }^{151}$ S. Jin, M. McCormack, T. H. Tiefel, and R. Ramesh, J. Appl. Phys. 76, 6929 (1994).

${ }^{152}$ X. D. Wu, R. C. Dye, R. E. Muenchausen, S. R. Foltyn, M. Maley, A. D. Rollett, A. R. Garcia, and N. S. Nogar, Appl. Phys. Lett. 58, 2165 (1991).

${ }^{153}$ J. C. Jiang, X. Q. Pan, and C. L. Chen, Appl. Phys. Lett. 72, 909 (1998).

${ }^{154}$ J. C. Jiang and X. Q. Pan, J. Appl. Phys. 89, 6365 (2001).

${ }^{155}$ C. D. Theis, J. Yeh, D. G. Schlom, M. E. Hawley, G. W. Brown, J. C. Jiang, and X. Q. Pan, Appl. Phys. Lett. 72, 2817 (1998).

${ }^{156}$ X. Q. Pan, J. C. Jiang, C. D. Theis, and D. G. Schlom, Appl. Phys. Lett. 83, 2315 (2003).

${ }^{157}$ Y. Barad, J. Lettieri, C. D. Theis, D. G. Schlom, V. Gopalan, J. C. Jiang, and X. Q. Pan, J. Appl. Phys. 89, 1387 (2001).

${ }^{158}$ J. C. Jiang, Y. Lin, C. L. Chen, C. W. Chu, and E. I. Meletis, J. Appl. Phys. 91, 3188 (2002).
${ }^{159}$ C. L. Chen, H. H. Feng, Z. Zhang, A. Brazdeikis, F. A. Miranda, F. W. Van Kewls, R. R. Romanofsky, Z. J. Huang, Y. Liou, W. K. Chu, and C. W. Chu, Appl. Phys. Lett. 75, 412 (1999).

${ }^{160}$ Z. Yuan, Y. Lin, J. Weaver, X. Chen, C. L. Chen, G. Subramanyam, J. C. Jiang, and E. I. Meletis, Appl. Phys. Lett. 87, 152901 (2005).

${ }^{161}$ M. Liu, C. R. Ma, G. Collins, J. Liu, C. L. Chen, L. Shui, H. Wang, C. Dai, Y. Lin, J. He, J. C. Jiang, E. I. Meletis, and Q. Y. Zhang, Cryst. Growth Des. 10, 4221 (2010).

${ }^{162}$ J. He, J. C. Jiang, E. I. Meletis, M. Liu, G. Collins, C. R. Ma, C. L. Chen, and A. Bhalla, Integr. Ferroelectr. 131, 72 (2011).

${ }^{163}$ J. He, J. C. Jiang, M. Liu, J. Liu, G. Collins, C. R. Ma, C. L. Chen, A. Bhalla, and E. I. Meletis, Philos. Mag. Lett. 91, 361 (2011).

${ }^{164}$ J. He, J. C. Jiang, E. I. Meletis, J. Liu, G. Collins, C. L. Chen, and A. Bhalla, Philos. Mag. Lett. 89, 493 (2009).

${ }^{165}$ G. Y. Wang, S. W. Liu, T. Wu, X. G. Luo, C. H. Wang, J. C. Jiang, X. H. Chen, and C. L. Chen, Solid State Commun. 144, 454 (2007).

${ }^{166}$ J. C. Jiang, E. I. Meletis, C. L. Chen, Y. Lin, Z. Zhang, and W. K. Chu, Philos. Mag. Lett. 84, 443 (2004).

${ }^{167}$ T. Hungria, J. Galy, and A. Castro, Adv. Eng. Mater. 11, 615 (2009).

${ }^{168}$ C. H. Lee, N. D. Orloff, T. Birol, Y. Zhu, V. Goian, E. Rocas, R. Haislmaier, E. Vlahos, J. A. Mundy, L. F. Kourkoutis, Y. Nie, M. D. Biegalski, J.-S. Zhang, M. Bernhagen, N. A. Bedenek, Y. Kim, J. D. Brock, R. Uecker, X. X. Xi, V. Gopalan, D. Nuzhnyy, S. Kamba, D. A. Muller, I. Takeuchi, J. C. Booth, C. J. Fennie, and D. G. Schlom, Nature 502, 532 (2013).

${ }^{169}$ J. C. Jiang, X. Q. Pan, W. Tian, C. D. Theis, and D. G. Schlom, Appl. Phys. Lett. 74, 2851 (1999)

${ }^{170}$ I. Bozovic, G. Logvenov, I. Belca, B. Narimbetov, and I. Sveklo, Phys. Rev. Lett. 89, 107001 (2002).

${ }^{171}$ M. Itoh, R. Wang, Y. Inaguma, T. Yamaguchi, Y. J. Shan, and T. Nakamura, Phys. Rev. Lett. 82, 3540 (1999)

${ }^{172}$ M. Liu, C. R. Ma, G. Collins, J. Liu, C. L. Chen, C. Dai, Y. Lin, L. Shui, H. Wang, J. He, J. C. Jiang, E. I. Meletis, Q. Y. Zhang, and M. W. Cole, Appl. Mater. Interfaces 4, 5761 (2012).

${ }^{173}$ J. C. Jiang, E. I. Meletis, L. L. Henry, K. I. Gnanasekar, and C. L. Chen, Nano Lett. 4, 741 (2004).

${ }^{174}$ J. He, J. C. Jiang, J. Liu, M. Liu, G. Collins, C. R. Ma, C. L. Chen, and E. I. Meletis, Thin Solid Films 519, 4371 (2011).

${ }^{175}$ M. Liu, J. Liu, C. Ma, G. Collins, C. L. Chen, A. D. Alemayehu, G. Subramanyam, J. He, J. Jiang, E. I. Meletis, and A. Bhalla, Cryst. Eng. Comm. 15(34), 6641 (2013).

${ }^{176}$ J. Lou, J. Wu, M. Liu, G. Wen, Y. Jin, and N. X. Sun, in IEEE Microwave Symposium (IMS) (2011), p. 1.

${ }^{177}$ N. Li, M. Liu, Z. Zhou, N. X. Sun, D. V. B. Murthy, G. Srinivasan, T. M. Klein, V. M. Petrov, and A. Gupta, Appl. Phys. Lett. 99, 192502 (2011).

${ }^{178}$ O. Obi, M. Liu, J. Lou, S. Stoute, X. Xing, N. X. Sun, J. Warzywoda, A. Sacco, Jr., D. E. Oates, and G. F. Dionne, J. Appl. Phys. 109, 07E527 (2011).

${ }^{179}$ F. Jona and G. Shirane, Ferroelectric Crystals (Pergamon Press, Oxford, New York, 1962).

${ }^{180}$ B. A. Strukov and A. P. Levanyuk, Ferroelectric Phenomena in Crystals (Springer-Verlag, Berlin, Heidelberg, 1998).

${ }^{181}$ G. A. Rossetti, Jr., "Thermodynamic theory," in Piezoelectricity: Evolution and Future of a Technology, edited by W. Heywang, K. Lubitz, and W. Wersing (Springer-Verlag, Berlin, 2008), pp. 493-516.

${ }^{182}$ Z.-G. Ban and S. P. Alpay, J. Appl. Phys. 93, 504 (2003).

${ }^{183}$ Z.-G. Ban and S. P. Alpay, J. Appl. Phys. 91, 9288 (2002).

${ }^{184}$ J. Zhang, M. W. Cole, and S. P. Alpay, J. Appl. Phys. 108, 054103 (2010).

${ }^{185}$ J. Zhang, C. V. Weiss, and S. P. Alpay, Appl. Phys. Lett. 99, 042902 (2011).

${ }^{186}$ A. L. Roytburd, S. Zhong, and S. P. Alpay, Appl. Phys. Lett. 87, 092902 (2005).

${ }^{187}$ S. Zhong, S. P. Alpay, and J. V. Mantese, Appl. Phys. Lett. 88, 132904 (2006).

${ }^{188}$ A. F. Devonshire, Philos. Mag. 40, 1040 (1949).

${ }^{189}$ A. F. Devonshire, Philos. Mag. 42, 1065 (1951).

${ }^{190}$ A. J. Bell and L. E. Cross, Ferroelectrics 59, 197 (1984).

${ }^{191}$ A. J. Bell, J. Appl. Phys. 89, 3907 (2001).

${ }^{192}$ Y. L. Li, L. E. Cross, and L. Q. Chen, J. Appl. Phys. 98, 064101 (2005).

${ }^{193}$ Y. L. Wang, A. K. Tagantsev, D. Damjanovic, N. Setter, V. K. Yarmarkin, A. I. Sokolov, and I. A. Lukyanchuk, J. Appl. Phys. 101, 104115 (2007). 
${ }^{194}$ J. J. Wang, P. P. Wu, X. Q. Ma, and L. Q. Chen, J. Appl. Phys. 108, 114105 (2010).

${ }^{195}$ V. E. Yurkevich and B. N. Rolov, Phys. Status Solidi B 52, 335 (1972).

${ }^{196}$ V. E. Yurkevich and B. N. Rolov, Phys. Status Solidi B 52, 683 (1972).

${ }^{197}$ G. A. Rossetti, Jr., A. G. Khachaturyan, G. Akcay, and Y. Ni, J. Appl. Phys. 103, 114113 (2008).

${ }^{198}$ A. A. Heitmann and G. A. Rossetti, Jr., Philos. Mag. 90, 71-87 (2010).

${ }^{199}$ A. A. Heitmann and G. A. Rossetti, Jr., Integr. Ferroelectr. 126, 155-165 (2011).

${ }^{200}$ V. V. Lemanov, E. P. Smirnova, P. P. Syrnikov, and E. A. Tarakanov, Phys. Rev. B 54, 3151 (1996).

${ }^{201}$ G. M. Yang, R. Jin, G. Xiao, C. Vittoria, V. G. Harris, and N. X. Sun, IEEE Trans. Antennas Propag. 57, 256 (2009).

${ }^{202}$ L. Q. Chen, J. Am. Ceram. Soc. 91(6), 1835 (2008).

${ }^{203}$ J. X. Zhang, Y. L. Li, D. G. Schlom, L. Q. Chen, F. Zavaliche, R. Ramesh, and Q. X. Jia, Appl. Phys. Lett. 90, 052909 (2007).
${ }^{204}$ Y. Ni and A. G. Khachaturyan, J. Appl. Phys. 102, 113506 (2007).

${ }^{205}$ J. M. Hu, G. Sheng, J. X. Zhang, C. W. Nan, and L. Q. Chen, J. Appl. Phys. 109, 123917 (2011).

${ }^{206}$ J. M. Hu, Z. Li, L. Q. Chen, and C. W. Nan, Adv. Mater. 24(21), 2869 (2012).

${ }^{207}$ J. M. Hu, Z. Li, L. Q. Chen, and C. W. Nan, Nat. Commun. 2, 553 (2011).

${ }^{208}$ Y. L. Li, S. Y. Hu, Z. K. Liu, and L. Q. Chen, Acta Mater. 50, 395 (2002).

${ }^{209}$ A. Bueno-Orovio, V. M. Perez-Garcia, and F. H. Fenton, SIAM J. Sci. Comput. 28, 886 (2006).

${ }^{210}$ K. Dayal and K. Bhattacharya, Acta Mater. 55, 1907 (2007).

${ }^{211}$ L. Yang and K. Dayal, J. Comput. Phys. 230, 7821 (2011).

${ }^{212}$ M. El-Naggar, K. Dayal, D. Goodwin, and K. Bhattacharya, J. Appl. Phys. 100, 114115 (2006).

${ }^{213}$ L. Yang and K. Dayal, Int. J. Fract. 174, 17 (2012).

${ }^{214}$ L. Yang and K. Dayal, J. Appl. Phys. 111, 014106 (2012).

${ }^{215}$ L. Yang and K. Dayal, Acta Mater. 60(19), 6457 (2012). 